LMU-ASC 45/05

MPP-2005-59

hep-th/0506090

\title{
Moduli Stabilization in Type IIB Orientifolds (I)
}

\author{
D. Lüst ${ }^{a, b}$, S. Reffert ${ }^{b}$, W. Schulgin ${ }^{b}$ and S. Stieberger ${ }^{a}$ \\ a Arnold-Sommerfeld-Center for Theoretical Physics, \\ Department für Physik, Ludwig-Maximilians-Universität München, \\ Theresienstraße 37, 80333 München, Germany \\ ${ }^{b}$ Max-Planck-Institut für Physik, \\ Föhringer Ring 6, 80805 München, Germany
}

\begin{abstract}
We discuss flux quantization and moduli stabilization in toroidal type IIB $\mathbf{Z}_{N^{-}}$or $\mathbf{Z}_{N} \times \mathbf{Z}_{M}$-orientifolds, focusing mainly on their orbifold limits. After presenting a detailed discussion of their moduli spaces and effective actions, we study the supersymmetric vacuum structure of these models and derive criteria for the existence of stable minima. Furthermore, we briefly investigate the models away from their orbifold points and comment on the microscopic origin of their non-perturbative superpotentials.
\end{abstract}




\section{Contents}

1. Introduction . . . . . . . . . . . . . . . . . . . . . . . . 3

2. Moduli space, tree-level effective action and 3-form fluxes in type IIB orientifolds 6 2.1. Type IIB orientifolds of toroidal orbifolds . . . . . . . . . . . . . . . . 6 2.2. Closed string moduli space _. . . . . . . . . . . . . . . . . . . 0

2.3. Background parameterization of geometric moduli in orientifolds of type IIB 10 2.4. Parametrization of coset spaces with more than three Kähler moduli . . . . 15 2.5. String-theoretical Kähler moduli $\mathcal{T}^{i}$ vs. field-theoretical fields $T^{i}$. . . . . 21 2.6. Three-form flux $G_{3}$ in $\mathbf{Z}_{N}$ and $\mathbf{Z}_{N} \times \mathbf{Z}_{M}$-orbifolds . . . . . . . . . . . 22 3. Vacuum structure of orientifolds in the orbifold limits . . . . . . . . . . 26 3.1. Supersymmetry conditions . . . . . . . . . . . . . . . . . . . . . 28 3.2. Orientifolds without complex structure modulus . . . . . . . . . . . . 31 3.3. Orientifolds with three Kähler moduli and fixed complex structure moduli . . 33 3.4. Orientifolds with five Kähler moduli and fixed complex structure moduli . . 35 3.5. Orientifolds with one untwisted complex structure modulus . . . . . . . . 37 3.6. Orientifolds with three untwisted complex structure moduli . . . . . . . . 40 3.7. Cubic superpotential

3.8. Open string moduli and soft-supersymmetry breaking terms . . . . . . . . 43 4. Non-perturbative superpotential, moduli stabilization and resolved orbifolds . . . 44 4.1. The non-perturbative superpotential . . . . . . . . . . . . . . . . . . 44 4.2. $M_{3}=T^{6} /\left(\mathbf{Z}_{N} \times \mathbf{Z}_{M}\right) \ldots \ldots \ldots$ 4.3. Moduli Stabilization in the resolved $\mathbf{Z}_{2} \times \mathbf{Z}_{2}$ orientifold revisited . . . . . . 52 4.4. Outlook: Resolved Orbifolds . . . . . . . . . . . . . 55 5. Conclusions . . . . . . . . . . . . . . . . . . . . . . . 57 Appendix A. Complex structures of $\mathbf{Z}_{N^{-}}$and $\mathbf{Z}_{N} \times \mathbf{Z}_{M^{-}}$orbifolds $\ldots . . . .58$

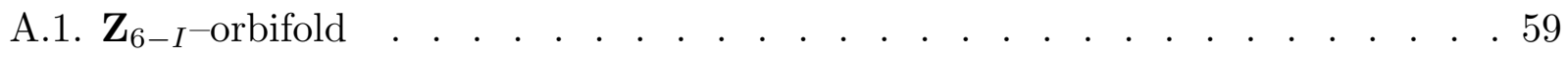

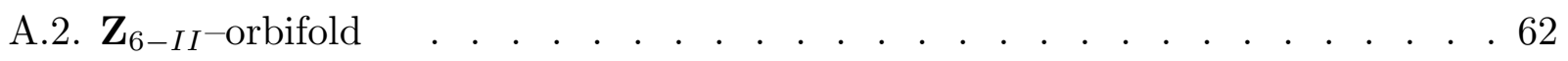




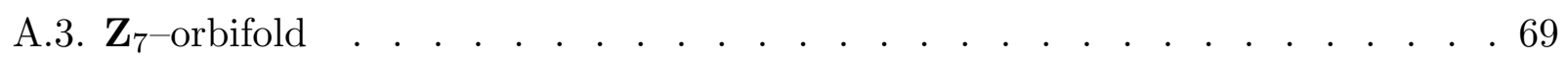

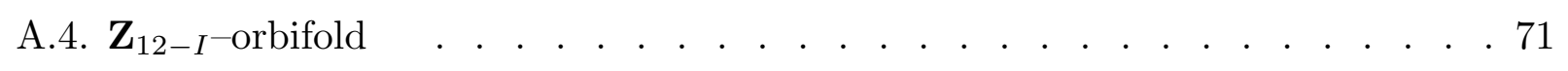

A.5. $\mathbf{Z}_{3} \times \mathbf{Z}_{3}$-orbifold . . . . . . . . . . . . . . . . . . . . . . . . 74

A.6. $\mathbf{Z}_{2} \times \mathbf{Z}_{6}$-orbifold . . . . . . . . . . . . . . . . . . . . . . . . . . . 75

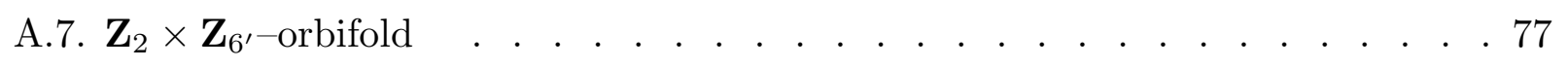

A.8. $\mathbf{Z}_{6} \times \mathbf{Z}_{6}$-orbifold . . . . . . . . . . . . . . . . . . . . . . . . . . 78

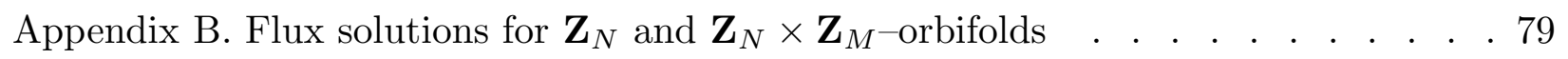

B.1. Invariant Fluxes . . . . . . . . . . . . . . . . . . . . . . . . . . . 80

B.2. $\mathbf{Z}_{3}$-orbifold . . . . . . . . . . . . . . . . . . . . . . . . 80

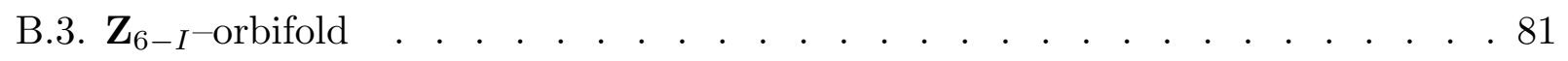

B.4. $\mathbf{Z}_{6-I I}$-orbifold . . . . . . . . . . . . . . . . . . . . . . . . . . . 82

B.5. $\mathbf{z}_{7}$-orbifold . . . . . . . . . . . . . . . . . . . . . . . . 85

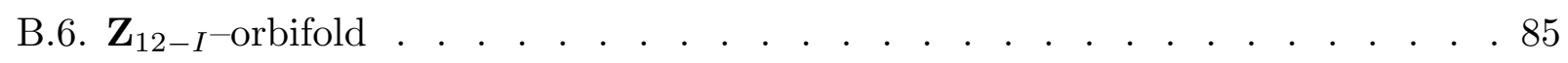

B.7. $\mathbf{Z}_{3} \times \mathbf{Z}_{3}$-orbifold . . . . . . . . . . . . . . . . . . . . . . . . . . . . . . 86

B.8. $\mathbf{Z}_{2} \times \mathbf{Z}_{6}$-orbifold . . . . . . . . . . . . . . . . . . . . . . . 87

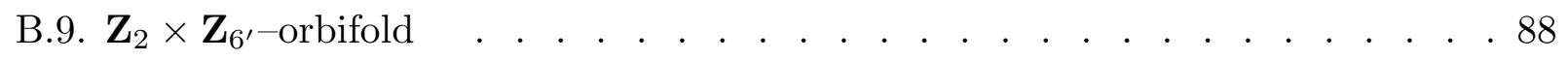

B.10. $\mathbf{Z}_{3} \times \mathbf{Z}_{6}$-orbifold . . . . . . . . . . . . . . . . . . . . . . . . 89

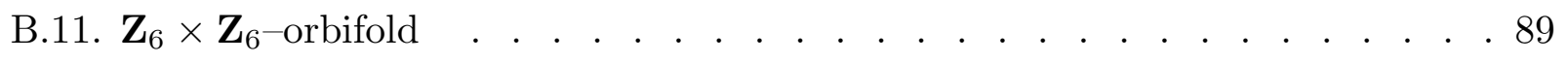

Appendix C. Cartan matrices of the relevant Lie groups . . . . . . . . . . . . 90

Appendix D. Anti-symmetric tensor and Kähler moduli for heterotic orbifolds . . . 90

D.1. Coset space $\frac{S U(2,2)}{S U(2) \times S U(2) \times U(1)} \times \frac{S U(1,1)}{U(1)}$. . . . . . . . . . . . . . . . . 91

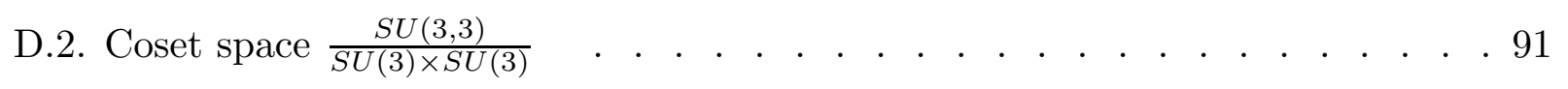




\section{Introduction}

Type $I I$-orientifold models are phenomenologically interesting for a number of reasons. First, they provide a viable framework to derive models that exhibit many features of the standard model of particle physics, such as non-Abelian gauge bosons, chiral fermions and family repetition (for some reviews see [1]). The present paper will deal with type $I I B$ orientifold compactification杬. In this context, the standard model fields are living on the world-volume of $D 3$-branes and/or $D 7$-branes which may intersect each other in the internal dimensions. Second, by adding closed string 3 -form fluxes via an effective superpotential $W_{\text {flux }}$ [8], many or even all of the geometric complex structure moduli plus the dilaton can be frozen [9, 10, 11]. At the same time, space-time supersymmetry may be spontaneously broken. Since the 3 -form fluxes arise already at string tree-level, it is possible to compute the pattern of the soft supersymmetry breaking parameters in the matter sector on the $D$-branes [12 18]. Last but not least, the proposal of KKLT [19] showed a promising possibility to freeze besides the complex structure moduli and the dilaton also all Kähler moduli through the effects of a non-perturbative superpotential $W_{\mathrm{np}}$. This superpotential can be induced by Euclidean D3-instantons and/or by gaugino condensation in some hidden gauge group sector. The scenario of KKLT has also far-reaching consequences in cosmology, since by lifting the potential by some other effects, e.g. by adding anti-branes, a small positive cosmological constant can be generated. Also string backgrounds with inflation in the early phase of the universe may have a similar origin [20]. As pointed out in Ref. [21], there exists a huge number of string ground states, called the string landscape. An observation, which motivates a statistical survey of all possible string vacua with fluxes [22] and also with $D$-branes [23].

Certainly being a very attractive scheme, there are still many open issues which should be addressed in the context of the KKLT proposal. The main question basically is, whether all moduli can be really stabilized in concrete and perhaps even semi-realistic orientifold models. Specifically, a semi-realistic model generically contains the following ingredients: after choosing a geometrical background space, in type IIB a (warped) compactification on $M_{4} \times X_{6}$, with $X_{6}$ being an orbifold or a Calabi-Yau space, an orientifold action is defined which reduces space-time supersymmetry from $\mathrm{N}=2$ down to $\mathrm{N}=1$ in four space-time dimensions. Orientifolds imply orientifold planes (O3/O7-planes) with negative $D$-brane charges, which require the presence of $D 3 / D 7$-branes and/or 3 -form fluxes, such that all tadpole cancelation conditions are satisfied, and $\mathrm{N}=1$ supersymmetry is preserved also in the open string sector of the theory. The next and also very important question for KKLT is whether Euclidean D3-instantons and/or gaugino condensation within the effective Yang-Mills theory on the $D 7$-branes can generate a non-vanishing non-perturbative

1 For some recent work on type $I I A$ orientifolds with fluxes and fixed moduli see [2] [7]. 
superpotential $W_{\mathrm{np}}$. The answer to this question is contained in the topology of the 4 -cycles (divisors) inside $X_{6}$, around which the $D 3 / D 7$-branes are wrapped. More specifically, for the Euclidean $D 3$-brane instantons, the number of fermionic zero modes living on the world volume of a D3-brane must be equal to two. For the wrapped D7-branes, the dynamics of open string non-Abelian gauge theory on a stack of $D 7$-branes must allow for a non-vanishing gaugino condensate. Whether all sufficient requirements for $W_{\mathrm{np}}$ are indeed satisfied depends however not only on the topology and geometry of the divisors inside $X_{6}$, but also on the chosen 3-form flux, which affects the number of fermionic zero modes on the $D 3$-branes as well as the open string spectrum on the D7-branes [24 30].

Therefore the tree-level superpotential $W_{\text {flux }}$ and the non-perturbative superpotential $W_{\text {np }}$ are not independent from each other but are rather intimately related. This observation really tells us that it is not useful to disentangle the vacuum structure due to the 3-form fluxes from the non-perturbative effects and vice versa.

In this paper, we will not discuss in very great detail the microscopic origin of the nonperturbative superpotential $W_{\mathrm{np}}$, but leave this for a future publication [31. (Recently a concrete type $I I B$ orientifold with all moduli fixed was constructed in [32].) Only a few remarks at the end of the paper will address this question. Our main emphasis in this work is the investigation of the moduli-spaces and the vacuum structure of type $I I B$ orientifold compactifications in their various toroidal orbifold limits. Hence we will simply assume the existence of a non-perturbative superpotential $W_{\mathrm{np}}$, which depends only on the untwisted Kähler moduli $T^{i}$. The effects of blowing up the orbifold or the presence of blowing up Kähler moduli will be neglected, respectively postponed for future work [31] (except for a short discussion at the end of the paper). So, $W_{\mathrm{np}}\left(T^{i}\right)$ can be viewed as being the truncation of a more complete superpotential that contains all Kähler moduli. Nevertheless, several interesting questions can be addressed within the orbifold framework.

The first issue which we will discuss is the careful parameterization of the moduli spaces of type $I I B$ orientifolds in the various orbifold limits. The proper $\mathrm{N}=1$ supergravity definition of the closed string Kähler moduli $T^{i}$ and complex structure moduli $U^{j}$ in terms of the background metrics $g_{i j}$ and Ramond 4 -form $C_{4}$ depends of course on the considered orbifold twists, but also on the underlying 6-dimensional group lattices. Moreover, the definition of the Kähler moduli of type $I I B$ orientifolds is different from the one in heterotic compactifications. We also determine the associated tree-level effective action, and the possible 3 -form fluxes in type $I I B$ orbifolds.

Second, in KKLT one assumes that the complex structure moduli are fixed by $W_{\text {flux }}$ alone and then are integrated out assuming that they are heavy. In particular, the assumption is made that the flux vacua are still given through 3-form fluxes which are still imaginary self dual $(I S D)$ and are of the Hodge types $G_{(2,1)}$ and $G_{(0,3)}$. We will see however that the inclusion of the additional non-perturbative effects in the superpotential besides the 3 -form fluxes has the effect of generic supersymmetric AdS ground-states 
being described by fluxes which are not anymore $I S D$ with only $G_{(2,1)}$ components, but will rather include also all $I A S D$ (imaginary anti self-dual) types as well (see also the discussion in [33]).

The third problem is related to the stability of the obtained supersymmetric vacua. Although stable AdS vacua generically allow for scalar fields with negative (mass) ${ }^{2}$, provided the masses still fulfill the Breitenlohner-Freedman bound [34], the KKLT framework only works if all (mass) ${ }^{2}$ eigenvalues of the fixed scalar fields are already positive in the AdS ground state. The reason for this stronger requirement is that otherwise, the uplift to a dS vacuum by adding a positive constant to the scalar potential would not work, i.e. would not lead to a stable dS ground state. However in concrete orientifold models, this stability criterion is far from being automatically satisfied, as already observed in [35]. We will discuss in which orbifold compactifications there is a chance to obtain stable AdS ground states with positive scalar (mass) ${ }^{2}$.

This paper is organized as follows. Section 2 deals with the complex structures and Kähler moduli of the different $\mathbf{Z}_{N}$ and $\mathbf{Z}_{N} \times \mathbf{Z}_{M}$-orbifolds. Here, only orbifolds are treated which lead to models that allow tadpole cancellation. Without introducing torsion or vector structure, this is possible for the following orbifolds: $\mathbf{Z}_{3}, \mathbf{Z}_{6-I}, \mathbf{Z}_{6-I I}, \mathbf{Z}_{12-I}, \mathbf{Z}_{2} \times \mathbf{Z}_{2}$, $\mathbf{Z}_{3} \times \mathbf{Z}_{3}, \mathbf{Z}_{2} \times \mathbf{Z}_{3}, \mathbf{Z}_{2} \times \mathbf{Z}_{6}, \mathbf{Z}_{2} \times \mathbf{Z}_{6^{\prime}}, \mathbf{Z}_{3} \times \mathbf{Z}_{6}$ and $\mathbf{Z}_{6} \times \mathbf{Z}_{6}$. As for many of the orbifold twists, several torus lattices are possible and the complex structure depends on the lattice, the complex structures and Kähler moduli of different lattices are worked out. We also discuss the closed string low energy effective action, namely the Kähler potential of the moduli fields. Then, we determine 3-form fluxes and the 3-form flux induced tree level superpotential. In particular, we give a general introduction to 3 -form fluxes and a list of all invariant flux components under all possible orbifold groups without discrete torsion. As a result, it turns out that only the $I A S D$-flux $G_{(3,0)}$ and the $I S D$ flux $G_{(0,3)}$ are generic for all orbifolds.

Section 3 will deal with the discussion of the (discrete) vacuum structure of the considered orientifold compactifications. Due to the presence of the 3 -form fluxes as well as the non-perturbative superpotential, all complex structure and Kähler moduli will be generically fixed at discrete values. We will see that due to the Kähler moduli dependent non-perturbative superpotential, the supersymmetry conditions on 3-form fluxes are changed compared to the pure flux case. Analyzing the stability properties of the associated scalar potential we will see that stable AdS ground states are not possible if the orbifold does not contain complex structure moduli regardless of the number of untwisted Kähler moduli present in $W_{\mathrm{np}}$. One needs at least one untwisted complex structure modulus $U$ for stability. We also comment on the integrating out procedure of the complex structure moduli, which can lead to inconsistent results, as it is the case for orbifolds with 
just one complex structure modulus. Furthermore, we briefly discuss open string moduli and soft-supersymmetry breaking terms in the presence of $W_{\mathrm{np}}$. We show that e.g. the ratio of gaugino masses after inclusion of $W_{\mathrm{np}}$ is given by the same quotient as without $W_{\text {np }}$ in a certain region in the parameter/moduli space.

Section 4 contains a preliminary discussion on the microscopic origin of $W_{\mathrm{np}}$. We will discuss a set of conditions for a non-vanishing $W_{\mathrm{np}}$, like that for the case of gaugino condensation in the hidden gauge sectors on stacks of $D 7$-branes, the associated $D 7$-brane divisors inside $X_{6}$ must not intersect each other. These conditions are most naturally met if the orbifold singularities are resolved. These resolved orbifolds, their vacuum structures and other related issues will then be the topic of the forthcoming publication [31].

Finally the two appendices $\mathrm{A}$ and $\mathrm{B}$ contain the technical details about the moduli spaces of the orientifolds, the definitions of the complex structure, the geometrical lattice structures which underlie the various orbifolds, and the possible flux solutions for the $\mathbf{Z}_{N}$ and $\mathbf{Z}_{N} \times \mathbf{Z}_{M}$-orbifolds. Appendix $\mathrm{C}$ gives the Cartan matrices of the relevant lattice Lie groups, and for completeness appendix D shows the definitions of the Kähler moduli on the heterotic side.

\section{Moduli space, tree-level effective action and 3-form fluxes in type $I I B$ orientifolds}

\subsection{Type IIB orientifolds of toroidal orbifolds}

We concentrate on orientifolds of type $I I B$ compactified on the toroidal orbifolds

$$
X_{6}=\frac{T^{6}}{\mathbf{Z}_{N}} \quad, \quad X_{6}=\frac{T^{6}}{\mathbf{Z}_{N} \times \mathbf{Z}_{M}},
$$

with the orbifold groups $\Gamma=\mathbf{Z}_{N}$ and $\Gamma=\mathbf{Z}_{N} \times \mathbf{Z}_{M}$. To define the orbifold compactification $X_{6}$, we must specify the six-torus $T^{6}$ and the discrete point group $\Gamma$. We will restrict ourselves to orbifolds with Abelian point group without discrete torsion. The point group element $\theta$ can then be written as $\theta=\exp \left[2 \pi i\left(v^{1} M^{12}+v^{2} M^{34}+v^{3} M^{56}\right)\right]$, where the $M^{i j}$ are the generators of the Cartan sub-algebra and $0 \leq\left|v^{i}\right|<1, i=1,2,3$. To obtain $\mathrm{N}=2$ supersymmetry, the point group $\Gamma$ must be a subgroup of $S U(3)$. This gives us $\pm v^{1} \pm v^{2} \pm v^{3}=0$. This condition together with the requirement that $\Gamma$ must act crystallographically on the lattice specified by $T^{6}$ leads to $\Gamma$ being either $\mathbf{Z}_{N}$ with $N=$ $3,4,6,7,8,12$ or $\mathbf{Z}_{M} \times \mathbf{Z}_{N}$ with $N$ a multiple of $M$ and $N=2,3,4 . \mathbf{Z}_{6}, \mathbf{Z}_{8}$ and $\mathbf{Z}_{12}$ have two inequivalent embeddings in $S O(6)$. We will use the standard embeddings, as given e.g. in 36.

To obtain an $\mathrm{N}=1$ (closed) string spectrum, one introduces an orientifold projection $\Omega I_{n}$, with $\Omega$ describing a reversal of the orientation of the closed string world-sheet and 
$I_{n}$ a reflection of $n$ internal coordinates. For $\Omega I_{n}$ to represent a symmetry of the original theory, $n$ has to be an even integer in type $I I B$. Generically, this projection produces orientifold fixed planes $\left[O(9-n)\right.$-planes], placed at the orbifold fixed points of $T^{6} / I_{n}$. They have negative tension, which has to be balanced by introducing positive tension objects. Candidates for the latter may be collections of $D(9-n)$-branes and/or non-vanishing three-form fluxes $H_{3}$ and $C_{3}$. The orbifold group $\Gamma$ mixes with the orientifold group $\Omega I_{n}$. As a result, if the group $\Gamma$ contains $\mathbf{Z}_{2}$-elements $\theta$, which leave one complex plane fixed, we obtain additional $O(9-|n-4|)-$ or $O(3+|n-2|)$-planes from the element $\Omega I_{n} \theta$.

In the following, only the two cases of $n=6$ (O3-plane) and $n=2(O 7$-planes) will be relevant to us. Without introducing torsion or vector structure, tadpoles may be completely cancelled by adding $D 3 / D 7$-branes, provided the orbifold twist $\Gamma$ is $\mathbf{Z}_{3}, \mathbf{Z}_{6-I}, \mathbf{Z}_{6-I I}, \mathbf{Z}_{7}$ or $\mathbf{Z}_{12-I}$ [37]. Furthermore, from the $\mathbf{Z}_{N} \times \mathbf{Z}_{M}$-orbifolds, only the twists $\mathbf{Z}_{2} \times \mathbf{Z}_{2}, \mathbf{Z}_{3} \times$ $\mathbf{Z}_{3}, \mathbf{Z}_{6} \times \mathbf{Z}_{6}, \mathbf{Z}_{2} \times \mathbf{Z}_{3}, \mathbf{Z}_{2} \times \mathbf{Z}_{6}, \mathbf{Z}_{2} \times \mathbf{Z}_{6}^{\prime}$ and $\mathbf{Z}_{3} \times \mathbf{Z}_{6}$ allow for tadpole cancellation in the above setup [38]. This is to be contrasted with type IIA intersecting $D 6$-brane constructions, where it has been recently shown that essentially all orbifold groups $\Gamma$ allow for tadpole cancellation due to the appearance of only untwisted and $\mathbf{Z}_{2}$-twisted sector tadpoles [39].

\subsection{Closed string moduli space}

The geometry of the orbifold $X_{6}$ is described by $h_{(1,1)}\left(X_{6}\right)$ Kähler moduli $\mathcal{T}^{i}$ and $h_{(2,1)}\left(X_{6}\right)$ complex structure moduli $\mathcal{U}^{i}$, which split into twisted and untwisted moduli. In the following, the dimension of the latter is denoted by $h_{(1,1)}^{\text {untw. }}\left(X_{6}\right)$ and $h_{(2,1)}^{\text {untw. }}\left(X_{6}\right)$, respectively. In addition, there is the complex dilaton field $S$ :

$$
S=i C_{0}+e^{-\phi_{10}}
$$

with $\phi_{10}$ the dilaton field and $C_{0}$ the Ramond scalar in $D=10$. The parameter space of $S$ is locally spanned by the coset

$$
\mathcal{M}_{S}=\frac{S U(1,1)}{U(1)}
$$

Furthermore, we have: $e^{-\phi_{10}}=e^{-\phi_{4}} \operatorname{Vol}\left(X_{6}\right)^{-1 / 2}$, with $\operatorname{Vol}\left(X_{6}\right)$ the volume of the compactification manifold $X_{6}$.

Without $D$-brane moduli, locally the closed string moduli space $\mathcal{M}$ is a direct product

of the complex dilaton field $S$, the Kähler $\mathcal{M}_{K}$ and complex structure moduli $\mathcal{M}_{C S}$, 40 (see also Refs. [41]):

$$
\mathcal{M}=\mathcal{M}_{S} \otimes \mathcal{M}_{K} \otimes \mathcal{M}_{C S}
$$


The Kähler metric for the moduli space $\mathcal{M}$ derives from the Kähler potential

$$
\begin{aligned}
K & =-\ln (S+\bar{S})-2 K_{\mathcal{K}}-K_{\mathcal{C S}} \\
& =-\ln (S+\bar{S})-2 \ln \operatorname{Vol}\left(X_{6}\right)-\ln \left(-i \int_{X_{6}} \Omega_{3} \wedge \bar{\Omega}_{3}\right),
\end{aligned}
$$

with $\Omega_{3}$ the holomorphic 3-form of the manifold $X_{6}$ and $\operatorname{Vol}\left(X_{6}\right)$ its volume. Above $K_{\mathcal{K}}$ represents the Kähler potential for the Kähler moduli $\mathcal{T}^{i}$ and $K_{\mathcal{C S}}$ the corresponding Kähler potential for the complex structure moduli $\mathcal{U}^{j}$.

Depending on the numbers $h_{(1,1)}^{\text {untw. }}, h_{(2,1)}^{\text {untw. }}$ of untwisted Kähler $\mathcal{T}^{i}$ and complex structure moduli $\mathcal{U}^{j}$, the generic (untwisted) moduli spaces $\mathcal{M}_{\mathcal{K}}, \mathcal{M}_{\mathcal{C S}}$ appearing in toroidal orbifold compactifications are described by the following six different cosets [42 45]:

$$
\begin{gathered}
h_{(1,1)}^{\text {untw. }}=3, \quad h_{(2,1)}^{\text {untw. }}=0,1,3: \quad \mathcal{M}_{\mathcal{K}}=\left(\frac{S U(1,1)}{U(1)}\right)^{3}, \mathcal{M}_{\mathcal{C S}}=\left(\frac{S U(1,1)}{U(1)}\right)^{h_{(2,1)}^{\text {untw. }}}, \\
h_{(1,1)}^{\text {untw. }}=5, \quad h_{(2,1)}^{\text {untw. }}=0,1: \quad \mathcal{M}_{\mathcal{K}}=\frac{S U(2,2)}{S U(2) \times S U(2) \times U(1)} \times\left(\frac{S U(1,1)}{U(1)}\right), \\
\mathcal{M}_{\mathcal{C S}}=\left(\frac{S U(1,1)}{U(1)}\right)^{h_{(2,1)}^{\text {untw. }}}, \\
h_{(1,1)}^{\text {untw. }}=9 \quad, \quad h_{(2,1)}^{\text {untw. }}=0: \quad \mathcal{M}_{\mathcal{K}}=\frac{S U(3,3)}{S U(3) \times S U(3) \times U(1)} .
\end{gathered}
$$

The numbers $h_{(1,1)}^{\text {twist. }}, h_{(2,1)}^{\text {twist. }}$ depend both on the orbifold group $\Gamma$ and the underlying torus lattice $T^{6}$ [46]. A list of representative orbifold examples, relevant to us in the following, is given in Table 1 and 2 .

\begin{tabular}{|l|l|l|l|l|l|l|}
\hline $\mathbf{Z}_{N}$ & Twist $\Gamma$ & Lattice $T^{6}$ & $h_{(1,1)}^{\text {untw. }}$ & $h_{(2,1)}^{\text {untw. }}$ & $h_{(1,1)}^{\text {twist. }}$ & $h_{(2,1)}^{\text {twist. }}$ \\
\hline $\mathbf{Z}_{3}$ & $\left(Z_{3}^{(2)}, Z_{3}^{(2)}, Z_{3}^{(2)}\right)$ & $S U(3)^{3}$ & 9 & 0 & 27 & 0 \\
$\mathbf{Z}_{6-I}$ & $\left(Z_{3}^{(2)}, Z_{6}^{(2)}, Z_{6}^{(2)}\right)$ & $S U(3) \times G_{2}^{2}$ & 5 & 0 & 24 & 5 \\
$\mathbf{Z}_{6-I}$ & $\left(Z_{6}^{(2)}, Z_{3}^{(4)}\right)$ & $G_{2} \times S U(3)_{b}^{2}$ & 5 & 0 & 20 & 1 \\
$\mathbf{Z}_{6-I I}$ & $\left(Z_{2}^{(1)}, Z_{2}^{(1)}, Z_{3}^{(2)}, Z_{6}^{(2)}\right)$ & $S U(2)^{2} \times S U(3) \times G_{2}$ & 3 & 1 & 32 & 10 \\
$\mathbf{Z}_{6-I I}$ & $\left(Z_{2}^{(1)}, Z_{3}^{(2)}, Z_{6}^{(3)}\right)$ & $S U(3) \times S O(8)$ & 3 & 1 & 26 & 4 \\
$\mathbf{Z}_{6-I I}$ & $\left(Z_{2}^{(1)}, Z_{2}^{(1)}, Z_{3}^{(4)}\right)$ & $S U(2)^{2} \times S U(3) \times S U(3)_{\sharp}$ & 3 & 1 & 28 & 6 \\
$\mathbf{Z}_{6-I I}$ & $\left(Z_{2}^{(1)}, Z_{6}^{(5)}\right)$ & $S U(2) \times S U(6)$ & 3 & 1 & 22 & 0 \\
$\mathbf{Z}_{7}$ & $\left(Z_{7}^{(6)}\right)$ & $S U(7)$ & 3 & 0 & 21 & 0 \\
$\mathbf{Z}_{12-I}$ & $\left(Z_{3}^{(2)}, Z_{12}^{(4)}\right)$ & $S U(3) \times F_{4}$ & 3 & 0 & 26 & 5 \\
$\mathbf{Z}_{12-I}$ & $\left(Z_{12}^{(6)}\right)$ & $E_{6}$ & 0 & 22 & 1 \\
\hline
\end{tabular}

Table 1: Twists, lattices and Hodge numbers for $\mathbf{Z}_{N}$ orbifolds. 


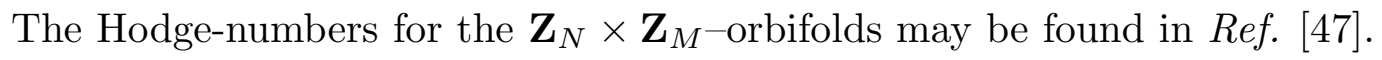

\begin{tabular}{|l|l|l|l|l|l|l|}
\hline $\mathbf{Z}_{N} \times \mathbf{Z}_{M}$ & $\theta$ & $\omega$ & $h_{(1,1)}^{\text {untw. }}$ & $h_{(2,1)}^{\text {untw. }}$ & $h_{(1,1)}^{\text {twist. }}$ & $h_{(2,1)}^{\text {twist. }}$ \\
\hline $\mathbf{Z}_{2} \times \mathbf{Z}_{2}$ & $\frac{1}{2}(1,0,-1)$ & $\frac{1}{2}(0,1,-1)$ & 3 & 3 & 48 & 0 \\
$\mathbf{Z}_{3} \times \mathbf{Z}_{3}$ & $\frac{1}{3}(1,0,-1)$ & $\frac{1}{3}(0,1,-1)$ & 3 & 0 & 81 & 0 \\
$\mathbf{Z}_{6} \times \mathbf{Z}_{6}$ & $\frac{1}{6}(1,0,-1)$ & $\frac{1}{6}(0,1,-1)$ & 3 & 0 & 81 & 0 \\
$\mathbf{Z}_{3} \times \mathbf{Z}_{6}$ & $\frac{1}{3}(1,0,-1)$ & $\frac{1}{6}(0,1,-1)$ & 3 & 0 & 70 & 1 \\
$\mathbf{Z}_{2} \times \mathbf{Z}_{3}$ & $\frac{1}{2}(1,0,-1)$ & $\frac{1}{3}(0,1,-1)$ & 3 & 1 & 32 & 10 \\
$\mathbf{Z}_{2} \times \mathbf{Z}_{6}$ & $\frac{1}{2}(1,0,-1)$ & $\frac{1}{6}(0,1,-1)$ & 3 & 1 & 48 & 2 \\
$\mathbf{Z}_{2} \times \mathbf{Z}_{6^{\prime}}$ & $\frac{1}{2}(1,0,-1)$ & $\frac{1}{6}(1,1,-2)$ & 3 & 0 & 33 & 0 \\
\hline
\end{tabular}

Table 2: Twists $\theta, \omega$ and Hodge numbers of $\mathbf{Z}_{N} \times \mathbf{Z}_{M}$ orbifolds.

The corresponding Kähler potentials for the spaces (2.6) are known from heterotic string compactifications [43]:

$$
\begin{gathered}
h_{(1,1)}^{\text {untw. }}=3, \quad h_{(2,1)}^{\text {untw. }}=0,1,3: \quad K_{\mathcal{K}}=-\sum_{i=1}^{3} \ln \left(\mathcal{T}^{i}+\overline{\mathcal{T}}^{i}\right), K_{\mathcal{C} \mathcal{S}}=-\sum_{j=1}^{h_{(2,1)}^{\text {untw. }}} \ln \left(\mathcal{U}^{j}+\overline{\mathcal{U}}^{j}\right), \\
h_{(1,1)}^{\text {untw. }}=5, h_{(2,1)}^{\text {untw. }}=0,1: \quad K_{\mathcal{K}}=-\ln \operatorname{det}\left(\mathcal{T}^{i j}+\overline{\mathcal{T}}^{i j}\right)-\ln \left(\mathcal{T}^{5}+\overline{\mathcal{T}}^{5}\right), \\
K_{\mathcal{C S}}=-\sum_{j=1}^{h_{(2,1)}^{\text {untw. }}} \ln \left(\mathcal{U}^{j}+\overline{\mathcal{U}}^{j}\right), \\
h_{(1,1)}^{\text {untw. }}=9, \quad h_{(2,1)}^{\text {untw. }}=0: \quad K_{\mathcal{K}}=-\ln \operatorname{det}\left(\mathcal{T}^{i j}+\overline{\mathcal{T}}^{i j}\right) .
\end{gathered}
$$

What is less known is the parameterization of the moduli fields $\mathcal{T}^{i}, \mathcal{U}^{i}$ in terms of the data of the torus, i.e. the real metric $g$ and the discrete symmetries of the underlying effective field theory. It is this aspect among others we shall elaborate in more detail in this section.

There is one important difference when compactifing the heterotic and type IIB string on the same six-manifold $X_{6}$. In the heterotic string, the complexification of the Kähler moduli $\mathcal{T}^{i}$ is achieved through the Neveu-Schwarz antisymmetric tensor $B_{2}$, while in the orientfolds we discuss here, this is accomplished with the Ramond 4-form $C_{4}$. Moreover, while the string-theoretical moduli fields $\mathcal{T}^{i}$ define proper complex scalars of chiral $\mathrm{N}=1$ multiplets in $D=4$ heterotic compactifications, they do not enjoy this property in type $I I B$ orientifolds. More precisely, in type $I I B$ the axionic part of the complexified Kähler 
modulus $\mathcal{T}^{i}$ is given by some internal component of the Ramond 4 -form $C_{4}$, i.e. the 4cycle integral $\int_{C_{i}} C_{4}$, while for the heterotic compactification on the same manifold $X_{6}$, the Kähler moduli are complexified with some internal part of the $N S 2$-form $B_{2}$, i.e. $\int_{C_{j}} B_{2}$, with some 2-cycle $C_{j}$. Since $h_{(2,2)}\left(X_{6}\right)=h_{(1,1)}\left(X_{6}\right)$, from the cohomological point of view, there is not much difference, as the 2 -form $\omega_{i}$, which appears in the expansion of $B_{2}$, is the Poincaré dual of the 4 -cycle $C_{i}$. An other peculiarity in type $I I B$ orientifold compactifications with wrapped $D 7$-branes is that the Kähler moduli $\mathcal{T}^{i}$ following from the geometry of the manifold $X_{6}$ do not represent scalars of chiral $\mathrm{N}=1$ multiplets in $D=4$. One has to define new moduli $T^{i}$, which refer to the underlying effective field theory and lead to the correct effective field theory description. In fact, a quite general formula may be given, which relates the $h_{(1,1)}$ string theoretical moduli fields $\mathcal{T}^{i}$ to their field-theoretical analogs $T^{i}$ (for more details $c f$. subsection 2.5):

$$
T^{i}=\frac{\partial}{\partial \operatorname{Re}\left(\mathcal{T}^{i}\right)} \operatorname{Vol}\left(X_{6}\left(\mathcal{T}^{j}\right)\right)+i \int_{C_{i}} C_{4}
$$

Here, $\operatorname{Vol}\left(X_{6}\left(\mathcal{T}^{j}\right)\right)$ is the volume (in string units) of the internal manifold $X_{6}$ expressed in terms of the Kähler moduli $\mathcal{T}^{j}$, defined in type $I I B$ on $X_{6}$.

As we may see from the list (2.6), the complex structure moduli space is much simpler, as this space only consists of factors of $\frac{S U(1,1)}{U(1)}$. Furthermore, in many of the orbifold examples, the complex structure moduli $\mathcal{U}^{i}$ are fixed through the orbifold twist, i.e. $h_{(2,1)}^{\text {untw. }}=0$. Only in the case when the orbifold has $\mathbf{Z}_{2}$-subelements, some $\mathcal{U}^{i}$ remain unfixed. Except for the twist $\mathbf{Z}_{2} \times \mathbf{Z}_{2}$, there may only exist one such $\mathbf{Z}_{2}$-element in order to preserve $\mathrm{N}=1$ supersymmetry in $D=4$. Hence, for $\mathbf{Z}_{2} \times \mathbf{Z}_{2}$ we have $h_{(2,1)}^{\text {untw. }}=3$, while all other orbifolds with $\mathbf{Z}_{2}$-elements have $h_{(2,1)}^{\text {untw. }}=1$. On the other hand, in type $I I B$ orientifolds the complex structure moduli $\mathcal{U}^{i}$ following from the string background $X_{6}$ already describe scalars $U^{i}$ of $\mathrm{N}=1$ chiral multiplets in $D=4$. Hence, we have:

$$
U^{i}=\mathcal{U}^{i} \quad, \quad i=1, \ldots, h_{(2,1)}^{\text {untw. }}
$$

\subsection{Background parameterization of geometric moduli in orientifolds of type IIB}

For the above orbifolds (with $(-1)^{F_{L}} I_{6} \Omega$ orientifold projection) we now want to find the parameterization of the (untwisted) Kähler $\mathcal{T}^{i}$ and complex structure moduli $\mathcal{U}^{i}$ in terms of their torus metric $g$ and their 4 -form background $C_{4}$. This classification is interesting on its own as it allows to study the modular symmetries of those compactifications. As we have already reported, in the type $I I B$ orientifolds we are discussing, the Kähler moduli $\mathcal{T}^{i}$ are complexified with components of the Ramond 4 -form $C_{4}$, while for heterotic compactification the Neveu-Schwarz 2-form $B_{2}$ is relevant. Since $h_{(2,2)}\left(X_{6}\right)=h_{(1,1)}\left(X_{6}\right)$, from the cohomological point of view, there is not much difference, as the 2-form $\omega_{i}$, 
which appears in the expansion of $B_{2}$, is the Poincaré dual of the 4 -cycle $C_{i}$. Hence, in the following, in particular in appendix A we shall very often derive benefits from this correspondence.

All we need to know about the lattices $T^{6}$ is contained in the Cartan matrix of the respective Lie algebra. The matrix elements of the Cartan matrix are defined as follows:

$$
A_{i j}=2 \frac{\left\langle e_{i}, e_{j}\right\rangle}{\left\langle e_{j}, e_{j}\right\rangle}
$$

where the $e_{i}$ are the simple roots. All Cartan matrices that will be needed here can be found in appendix D. The above orbifolds are all Coxeter orbifolds, i.e. the twist $\Gamma$ corresponds to the Coxeter element of the Lie algebra. It is given by successive Weyl reflections with respect to all simple roots: $Q=S_{1} S_{2} \ldots S_{\text {rank }}$, where the Weyl reflections are given by

$$
S_{i}(\mathbf{x})=\mathbf{x}-2 \frac{\left\langle\mathbf{x}, e_{i}\right\rangle}{\left\langle e_{i}, e_{i}\right\rangle} e_{i}
$$

Generalized Coxeter automorphisms are obtained by combining Weyl reflections with outer automorphisms, which are generated by transpositions of roots that are symmetries of the Dynkin diagram. $P_{i j}$ denotes the transposition of the $i$ 'th and $j$ 'th roots. The lattices marked with $b$ and $\sharp$ are realized as generalized Coxeter twists, the automorphism is in the first case $S_{1} S_{2} S_{3} S_{4} P_{36} P_{45}$ and in the second $S_{1} S_{2} S_{3} P_{34} S_{5} S_{6}$.

The orbifold twist $\Gamma$ may be represented by a matrix $Q_{i j}$, which rotates the six lattice basis vectors: $e_{i} \rightarrow Q_{j i} e_{j}$. Once we have determined the Coxeter element $Q$ via (2.11) and the Cartan matrix of the lattice in question, its metric $g$ can be obtained through the requirement that the orbifold twist must leave the scalar product invariant [48], i.e. :

$$
Q^{t} g Q=g
$$

We obtain the form of the metric in the lattice basis2 2 . The metric is conveniently parameterized via the lengths of the basis vectors and the angles between them: $\left\langle e_{i}, e_{j}\right\rangle=$ $R_{i} R_{j} \cos \theta_{i j}$. The form of the antisymmetric tensor $b$ is obtained in the same fashion, by solving

$$
Q^{t} b Q=b
$$

2 Speaking of the lattice basis is a bit misleading, as the orbifold twists generically allow more degrees of freedom in the lattice (i.e. Kähler and complex structure moduli) than the Lie group, which leaves only the overall normalization of the lattice vectors unfixed. So when we speak of the lattice basis we mean the basis that captures the extra degrees of freedom of the orbifold and not the basis of the root lattice. 
By now we also know the number of untwisted Kähler and complex structure moduli: We count the number of independent deformations $d$ allowed by the solutions of $Q^{t} g Q=g$ and $Q^{t} b Q=b$. The orbifold has $d_{b}$ untwisted Kähler moduli and $\frac{1}{2}\left(d_{g}-d_{b}\right)$ untwisted complex structure moduli.

To find the actual dependence of the Kähler and complex structure moduli on the degrees of freedom following from the above described parameterization $g, C_{4}$ (or $b$ ) in the lattice basis, one has to go into a complex basis $\left\{z^{i}\right\}_{i=1,2,3}$, where the twist $Q$ acts diagonally on the complex coordinates, i.e. $\Theta: d z^{i} \rightarrow e^{2 \pi i v^{i}} d z^{i}$, with the eigenvalues $v^{i}$ introduced after $E q$. (2.1). To find these complex coordinates we make the ansatz

$$
d z^{i}=a_{1}^{i} d x^{1}+a_{2}^{i} d x^{2}+a_{3}^{i} d x^{3}+a_{4}^{i} d x^{4}+a_{5}^{i} d x^{5}+a_{6}^{i} d x^{6}
$$

and solve the equation $Q^{t} d z^{i}=e^{2 \pi i v^{i}} d z^{i}$ which fixes $d z^{i}$ up to some constant factors. For convenience, we usually choose a normalization such that the first term comes with coefficient one. In addition, the ansatz (2.14) should yield a Hermitian metric, i.e. we require the identity: $d s^{2}=g_{i j} d x^{i} \otimes d x^{j}=g_{i \bar{j}} d z^{i} \otimes d \bar{z}^{j}$. After having introduced the complex coordinates $d z^{i}$, which define the complex structure moduli $\mathcal{U}^{i}$, we write down the Kähler form $J=g_{i \bar{j}} d z^{i} \wedge d \bar{z}^{j}$ and the anti-symmetric 4-form $C_{4}=\sum_{i=1}^{h_{(2,2)}\left(X_{6}\right)} c^{i} d_{i}$ (or the 2 -form $B_{2}=b_{i \bar{j}} d z^{i} \wedge d \bar{z}^{j} \equiv \sum_{i=1}^{h_{(1,1)}\left(X_{6}\right)} b^{i} \omega_{i}$ on the heterotic side). Here, the $\omega_{i}$ represent a basis of twist-invariant 2-forms of the real cohomology $H^{2}\left(X_{6}, \mathbf{Z}\right)$, while the $d_{i}$ supply a twist-invariant basis for $H^{4}\left(X_{6}, \mathbf{Z}\right)$. On the heterotic side, the Kähler moduli $\mathcal{T}^{i}$ are defined via the pairing $J+i B=\sum_{i=1}^{h_{(1,1)}\left(X_{6}\right)} \mathcal{T}^{i} \omega_{i}$. On the other hand, following the discussion after $E q$. (2.7), in type $I I B$ we may define the Kähler moduli $\mathcal{T}^{i}$ :

$$
\mathcal{T}^{i}=\int_{C_{2}^{i}} J+i \int_{C_{4}^{i}} C_{4} \quad, \quad i=1, \ldots, h_{(1,1)}\left(X_{6}\right),
$$

with the 2-cycle $C_{2}^{i}$ and the 4 -cycle $C_{4}^{i}$. The Poincare dual 2-form of $C_{4}^{i}$ is Hodge-dual to the Poincaré dual 4-form of $C_{2}^{i}$. This is way both cycles $C_{2}^{i}, C_{4}^{i}$ carry the same index. The first term $\int_{C_{2}^{i}} J$ of $\mathcal{T}^{i}$ describes the volume of the 2 -cycle $C_{2}^{i}$. In subsection 2.5 we shall relate the latter to the volume of the 4 -cycle $C_{4}^{i}$, which enters in the holomorphic moduli fields $T^{i}$ ( $c f . E q$. (2.8)).

As an illustrative example, we shall now work out the parameterization of the metric moduli of a $\mathbf{Z}_{N} \times \mathbf{Z}_{M}$-orbifold. All the $\mathbf{Z}_{N} \times \mathbf{Z}_{M}$-twists can be realized by automorphisms on either $S U(3)$ or $G_{2}$-sublattices. For $\mathbf{Z}_{N} \times \mathbf{Z}_{M}$-orbifolds, we are looking for a complex structure that is compatible with the two different twists as well as the combination of the two. The constraints are more stringent than in a simple $\mathbf{Z}_{N}$-twist, therefore the 
resulting lattices have less degrees of freedom. While the case $\mathbf{Z}_{3} \times \mathbf{Z}_{6}$ is treated here, for $\mathbf{Z}_{3} \times \mathbf{Z}_{3}, \mathbf{Z}_{2} \times \mathbf{Z}_{6}, \mathbf{Z}_{2} \times \mathbf{Z}_{6^{\prime}}$ and $\mathbf{Z}_{6} \times \mathbf{Z}_{6}$ the reader is referred to appendix A. The $\mathbf{Z}_{3} \times \mathbf{Z}_{6}$-orbifold has the following two twists:

$$
\begin{aligned}
& Q_{1}: z^{i} \longrightarrow e^{2 \pi i v_{1}^{i}} z^{i} \quad, \quad v_{1}^{1}=\frac{1}{3}, v_{1}^{2}=0, v_{1}^{3}=-\frac{1}{3} \\
& Q_{2}: z^{i} \longrightarrow e^{2 \pi i v_{2}^{i}} z^{i} \quad, \quad v_{2}^{1}=0, v_{2}^{2}=\frac{1}{6}, v_{2}^{3}=-\frac{1}{6} .
\end{aligned}
$$

The combined twist $Q_{3}=Q_{2} Q_{1}$ is a $\mathbf{Z}_{6-I I^{-}}$twist:

$$
Q_{3}: z^{i} \longrightarrow e^{2 \pi i v_{3}^{i}} z^{i} \quad, \quad v_{3}^{1}=\frac{2}{6}, v_{3}^{2}=\frac{1}{6}, v_{3}^{3}=-\frac{3}{6} .
$$

We know that the $\mathbf{Z}_{6-I I^{-}}$twist can live on $(S U(2))^{2} \times S U(3) \times G_{2}$, its action on the root lattice is worked out in the appendix A. A two-dimensional $\mathbf{Z}_{3}$-twist lives on the $S U(3)-$ lattice, while a two-dimensional $\mathbf{Z}_{6}$-twist lives on the $G_{2}$-lattice. Knowing all this, it is possible to construct the following twists:

$$
\begin{aligned}
& Q_{1} e_{1}=e_{2}, \quad Q_{1} e_{2}=-e_{1}-e_{2}, \quad Q_{1} e_{3}=e_{3}, \quad Q_{1} e_{4}=e_{4}, \\
& Q_{1} e_{5}=e_{5}+3 e_{6}, \quad Q_{1} e_{6}=-e_{5}-2 e_{6}, \\
& Q_{2} e_{1}=e_{1}, \quad Q_{2} e_{2}=e_{2}, \quad Q_{2} e_{3}=2 e_{3}+3 e_{4}, \quad Q_{2} e_{4}=-e_{3}-e_{4}, \\
& Q_{2} e_{5}=2 e_{5}+3 e_{6}, \quad Q_{2} e_{6}=-e_{5}-e_{6} .
\end{aligned}
$$

The twist on $e_{5}, e_{6}$ in $Q_{1}$ is minus the anti-twist of the usual Coxeter-twist on $G_{2}$, while all other twists are the usual $\mathbf{Z}_{2}, \mathbf{Z}_{3}$ and $\mathbf{Z}_{6}$-twists on their respective lattices. The twists reproduce the correct eigenvalues and the conditions $Q_{1}^{3}=1, Q_{2}^{6}=1$. The combined twist $Q_{3}$ has the form

$$
\begin{aligned}
& Q_{3} e_{1}=e_{2}, \quad Q_{3} e_{2}=-e_{1}-e_{2}, \\
& Q_{3} e_{3}=2 e_{3}+3 e_{4}, \quad Q_{3} e_{4}=-e_{3}-e_{4}, \\
& Q_{3} e_{5}=-e_{5}, \quad Q_{3} e_{6}=-e_{6},
\end{aligned}
$$

which is as just mentioned a $\mathbf{Z}_{6-I I}$-twist, namely the one on the lattice $S U(2)^{2} \times S U(3) \times$ $G_{2}$. As before, we require the metric to be invariant under all three twists, i.e. we impose the three conditions $Q_{i}^{T} g Q_{i}=g, \quad i=1,2,3$. This leads to the following solution:

$$
g=\left(\begin{array}{cccccc}
R_{1}^{2} & -\frac{1}{2} R_{1}^{2} & 0 & 0 & 0 & 0 \\
-\frac{1}{2} R_{1}^{2} & R_{1}^{2} & 0 & 0 & 0 & 0 \\
0 & 0 & R_{3}^{2} & -\frac{1}{2} R_{3}^{2} & 0 & 0 \\
0 & 0 & -\frac{1}{2} R_{3}^{2} & \frac{1}{3} R_{3}^{2} & 0 & 0 \\
0 & 0 & 0 & 0 & R_{5}^{2} & -\frac{1}{2} R_{5}^{2} \\
0 & 0 & 0 & 0 & -\frac{1}{2} R_{5}^{2} & \frac{1}{3} R_{5}^{2} .
\end{array}\right)
$$


This corresponds exactly to the metric of $S U(3) \times\left(G_{2}\right)^{2}$ without any extra degrees of freedom. The corresponding solution for $b$ has the form

$$
b=\left(\begin{array}{cccccc}
0 & b_{1} & 0 & 0 & 0 & 0 \\
-b_{1} & 0 & 0 & 0 & 0 & 0 \\
0 & 0 & 0 & b_{3} & 0 & 0 \\
0 & 0 & -b_{3} & 0 & 0 & 0 \\
0 & 0 & 0 & 0 & 0 & -b_{5} \\
0 & 0 & 0 & 0 & -b_{5} & 0
\end{array}\right) .
$$

We have three Kähler moduli while the complex structure is completely fixed. We find the following complex coordinates:

$$
\begin{aligned}
& d z^{1}=3^{1 / 4}\left(d x^{1}+e^{2 \pi i / 3} d x^{2}\right) \\
& d z^{2}=d x^{3}+\frac{1}{\sqrt{3}} e^{5 \pi i / 6} d x^{4} \\
& d z^{3}=d x^{5}+\frac{1}{\sqrt{3}} e^{-5 \pi i / 6} d x^{6}
\end{aligned}
$$

The invariant 2-forms in the real cohomology are simply $d x^{1} \wedge d x^{2}, d x^{3} \wedge d x^{4}$ and $d x^{5} \wedge d x^{6}$. The Kähler form in complex coordinates has the form

$$
-i J=\frac{1}{\sqrt{3}} R_{1}^{2} d z^{1} \wedge d \bar{z}^{1}+R_{3}^{2} d z^{1} \wedge d \bar{z}^{1}+R_{5}^{2} d z^{3} \wedge d \bar{z}^{3} .
$$

Expressed in the real cohomology, it takes the form

$$
J=\frac{\sqrt{3}}{2} R_{1}^{2} d x^{1} \wedge d x^{2}+\frac{1}{2 \sqrt{3}} R_{3}^{2} d x^{3} \wedge d x^{4}+\frac{1}{2 \sqrt{3}} R_{5}^{2} d x^{5} \wedge d x^{6} .
$$

So via the pairing $J+i B=\mathcal{T}^{i} \omega_{i}$ in the real cohomology, we find the three Kähler moduli to take the following form

$$
\begin{aligned}
\mathcal{T}^{1} & =\frac{\sqrt{3}}{2} R_{1}^{2}+i c_{1}, \\
\mathcal{T}^{2} & =\frac{1}{2 \sqrt{3}} R_{3}^{2}+i c_{2}, \\
\mathcal{T}^{3} & =\frac{1}{2 \sqrt{3}} R_{5}^{2}+i c_{3}
\end{aligned}
$$

after translating the heterotic components of $B_{2}$ into the respective components of the Ramond 4-form $C_{4}$, i.e. $c_{i} \simeq b_{i}$.

We shall in the following derive the Kähler moduli and complex structures for the $\mathbf{Z}_{N^{-}}$orbifolds, which allow for tadpole cancellation without introducing torsion or vector structure, i.e. $\mathbf{Z}_{7}, \mathbf{Z}_{6-I}, \mathbf{Z}_{6-I I}$ and $\mathbf{Z}_{12-I}$, in appendix A. The $\mathbf{Z}_{2} \times \mathbf{Z}_{2}$ and $\mathbf{Z}_{3}$-orbifolds are treated in the next subsection. 


\subsection{Parametrization of coset spaces with more than three Kähler moduli}

After having presented the general procedure of how to find the parameterization of the metric moduli in terms of the backgrounds $g, C_{4}$ (or $b$ in the heterotic case), in this subsection we shall demonstrate this on some selected orbifold examples which have more than three (untwisted) Kähler moduli. The simplest realization of the Kähler moduli spaces $\mathcal{M}_{\mathcal{K}}=\left(\frac{S U(1,1)}{U(1)}\right)^{3}, \mathcal{M}_{\mathcal{K}}=\frac{S U(2,2)}{S U(2) \times S U(2) \times U(1)} \times\left(\frac{S U(1,1)}{U(1)}\right)$ and $\mathcal{M}_{\mathcal{K}}=\frac{S U(3,3)}{S U(3) \times S U(3) \times U(1)}$, given in $E q$. (2.6), are the toroidal orbifolds $T^{6} / \mathbf{Z}_{2} \times \mathbf{Z}_{2}, T^{6} / \mathbf{Z}_{4}$ and $T^{6} / \mathbf{Z}_{3}$, respectively. Let us present the parameterization of their Kähler moduli in terms of their backgrounds $g, C_{4}$ for type $I I B$ orientifolds.

Kähler moduli space $\mathcal{M}_{\mathcal{K}}=\left(\frac{S U(1,1)}{U(1)}\right)^{3}$

This Kähler moduli space $\mathcal{M}_{\mathcal{K}}$ is realized e.g. in the $\mathbf{Z}_{2} \times \mathbf{Z}_{2}$ type IIB orientifold, with the twists $Q_{1}, Q_{2}$

$$
\begin{array}{ll}
Q_{1}: e_{1,2} \longrightarrow-e_{1,2} \quad, \quad e_{3,4} \longrightarrow-e_{4,5} \quad, \quad e_{5,6} \longrightarrow e_{5,6} \\
Q_{2}: e_{1,2} \longrightarrow-e_{1,2} \quad, \quad e_{3,4} \longrightarrow e_{4,5} \quad, \quad e_{5,6} \longrightarrow-e_{5,6}
\end{array}
$$

acting on the integral basis $\left\{e_{i}\right\}$ of the torus $T^{6}$. According to (2.12), the twists $Q_{1}, Q_{2}$ only allow for a factorizable lattice, i.e. $T^{6}$ being a direct product of three 2 -tori, i.e. $T^{6}=\otimes_{j=1}^{3} T^{2, j}$, with the metrics $g^{j}$. Each individual 2-torus $T^{2, j}$ has one Kähler modulus $\mathcal{T}^{j}$ and one complex structure modulus $\mathcal{U}^{j}$ describing the real parameters of the metric $g^{j}$. The complex structure moduli are given by:

$$
\mathcal{U}^{j}=\frac{1}{g_{11}^{j}}\left(\sqrt{\operatorname{det} g^{j}}+i g_{12}^{j}\right), \quad \text { with }: \quad g^{j}=\left(\begin{array}{cc}
g_{11}^{j} & g_{12}^{j} \\
g_{12}^{j} & g_{22}^{j}
\end{array}\right) .
$$

Some of these moduli may be fixed through the orbifold group resulting in $h_{(2,1)}^{\text {untw. complex }}$ structure moduli $U^{j}$ entering (2.5). The real part of the Kähler modulus $\mathcal{T}^{j}$ describes the size $\sqrt{\operatorname{det} g^{j}}$ of the subtorus $T^{2, j}$, i.e. $\operatorname{Re}\left(\mathcal{T}^{j}\right)=\sqrt{\operatorname{det} g^{j}}$. The twist-invariant 4 -form $C_{4}$ is given by

$$
C_{4}=c^{1} d x^{2} \wedge d y^{2} \wedge d x^{3} \wedge d y^{3}+c^{2} d x^{1} \wedge d y^{1} \wedge d x^{3} \wedge d y^{3}+c^{3} d x^{1} \wedge d y^{1} \wedge d x^{2} \wedge d y^{2}
$$

The Kähler modulus $\mathcal{T}^{j}$ is complexified with the internal part of the Ramond 4-form $C_{4}$, i.e. $\operatorname{Im}\left(\mathcal{T}^{j}\right)=\int_{T^{2, k} \times T^{2, l}} C_{4}=c^{j}$. Altogether, the three Kähler moduli $\mathcal{T}^{j}$ for the coset $\mathcal{M}_{\mathcal{K}}$ are:

$$
\mathcal{T}^{j}=i c^{j}+\sqrt{\operatorname{det} g^{j}} \quad, \quad j=1,2,3 .
$$


The type $I I B$ Kähler potential $K_{\mathcal{K}}$ is $(c f . E q .(2.7))$ :

$$
K_{\mathcal{K}}=-\ln \operatorname{Vol}\left(X_{6}\right)=-\sum_{i=1}^{3} \ln \left[\frac{1}{2}\left(\mathcal{T}^{i}+\overline{\mathcal{T}}^{i}\right)\right]
$$

According to $E q$. (2.8), the moduli $\mathcal{T}$ have to be translated into the holomorphic coordinates $T^{j}$. With $\operatorname{Vol}\left(X_{6}\right)=\operatorname{Re}\left(\mathcal{T}^{1}\right) \operatorname{Re}\left(\mathcal{T}^{2}\right) \operatorname{Re}\left(\mathcal{T}^{3}\right)$, we obtain [14]:

$$
T^{j}=i c^{j}+\operatorname{Re}\left(\mathcal{T}^{k}\right) \operatorname{Re}\left(\mathcal{T}^{l}\right),(j, k, l) \in \overline{(1,2,3)} .
$$

In terms of these holomorphic coordinates $T^{j}$ the Kähler potential (2.5) becomes:

$$
\kappa_{4}^{-2} K=-\ln (S+\bar{S})-\sum_{i=1}^{3} \ln \left[\frac{1}{2}\left(T^{i}+\bar{T}^{i}\right)\right]-\sum_{j=1}^{h_{(2,1)}^{\text {untw. }}} \ln \left(U^{j}+\bar{U}^{j}\right)
$$

$\underline{\text { Kähler moduli space } \mathcal{M}_{\mathcal{K}}=\frac{S U(2,2)}{S U(2) \times S U(2) \times U(1)} \otimes\left(\frac{S U(1,1)}{U(1)}\right)}$

Let us move on to the special Kähler moduli space $\mathcal{M}_{\mathcal{K}}$, which may be realized e.g. in the $\mathbf{Z}_{4}$-orbifold with the twist $v^{i}=\frac{1}{4}(1,1,-2)$. For concreteness we assume the twist to act block-diagonally within the six-torus $T^{6}$ and the latter to be factorizable into $T^{6}=T^{4} \otimes T^{2}$. Then the $\mathbf{Z}_{2}$-element of the twist entirely acts in the 2 -torus. The complex structure modulus for the latter is defined as in Eq. (2.27). The real part of the Kähler modulus $\mathcal{T}^{5}$ of the 2 -torus $T^{2}$ describes the volume of this torus. The complexified Kähler modulus $\mathcal{T}^{5}$ gives rise to the extra factor $\left(\frac{S U(1,1)}{U(1)}\right)$ of $\mathcal{M}_{\mathcal{K}}$ and will be given in a moment.

On the other hand, the parameterization of the four Kähler moduli $\mathcal{T}^{i j}, i, j=1,2$ in terms of the lattice properties of $T^{4}$ need some more work. According to (2.12) the orbifold twist $Q$, which acts through

$$
Q: e_{1} \longrightarrow e_{2} \quad, \quad e_{2} \longrightarrow-e_{1} \quad, \quad e_{3} \longrightarrow e_{4} \quad, \quad e_{4} \longrightarrow-e_{3}
$$

on the root basis $\left\{e_{i}\right\}$ of $T^{4}$ allows for the four metric components

$$
g=\left(\begin{array}{cccc}
g_{11} & 0 & g_{13} & -g_{14} \\
0 & g_{11} & g_{14} & g_{13} \\
g_{13} & g_{14} & g_{22} & 0 \\
-g_{14} & g_{13} & 0 & g_{22}
\end{array}\right)
$$

Following (2.14), we introduce complex structures and the complex coordinates $z^{1}, z^{2}$ :

$$
d z^{1}=\frac{1}{\sqrt{2}}\left(d x^{1}+i d y^{1}\right) \quad, \quad d z^{2}=\frac{1}{\sqrt{2}}\left(d x^{2}+i d y^{2}\right) .
$$


W.r.t the latter, the twist (2.33) acts diagonally, i.e. $\Theta: z^{1} \rightarrow i z^{1}, z^{2} \rightarrow i z^{2}$, with the twist eigenvalue $i$. In these complex coordinates the Kähler form $J=g_{i \bar{j}} d z^{i} \wedge d \bar{z}^{\bar{j}}$ becomes

$$
\begin{aligned}
-i J & =g_{11} d z^{1} \wedge d \bar{z}^{1}+g_{22} d z^{2} \wedge d \bar{z}^{2}+\operatorname{Re}\left(\mathcal{T}^{5}\right) d z^{3} \wedge d \bar{z}^{3} \\
& +\left(g_{13}-i g_{14}\right) d z^{1} \wedge d \bar{z}^{2}+\left(g_{13}+i g_{14}\right) d z^{2} \wedge d \bar{z}^{1}
\end{aligned}
$$

or expanded w.r.t the integer cohomology $H^{2}\left(X_{6}, \mathbf{Z}\right)$ :

$$
\begin{aligned}
J & =g_{11} d x^{1} \wedge d y^{1}+g_{22} d x^{2} \wedge d y^{2}+\operatorname{Re}\left(\mathcal{T}^{5}\right) d x^{3} \wedge d y^{3} \\
& +g_{14}\left(d x^{1} \wedge d x^{2}+d y^{1} \wedge d y^{2}\right)+g_{13}\left(d x^{1} \wedge d y^{2}-d y^{1} \wedge d x^{2}\right)
\end{aligned}
$$

The 4-form $C_{4}$ may be expanded w.r.t. the twist-invariant elements of the integral cohomolgy $H^{4}\left(T^{6}, \mathbf{Z}\right)$ :

$$
\begin{aligned}
C_{4} & =c_{1} d x^{2} \wedge d y^{2} \wedge d x^{3} \wedge d y^{3}+c_{2} d x^{1} \wedge d y^{1} \wedge d x^{3} \wedge d y^{3}+c_{5} d x^{1} \wedge d y^{1} \wedge d x^{2} \wedge d y^{2} \\
& -\frac{1}{2} c_{3}\left(d x^{1} \wedge d x^{2} \wedge d x^{3} \wedge d y^{3}+d y^{1} \wedge d y^{2} \wedge d x^{3} \wedge d y^{3}\right) \\
& +\frac{1}{2} c_{4}\left(d y^{1} \wedge d x^{2} \wedge d x^{3} \wedge d y^{3}-d x^{1} \wedge d y^{2} \wedge d x^{3} \wedge d y^{3}\right)
\end{aligned}
$$

which becomes:

$$
\begin{aligned}
C_{4} & =-c_{1} d z^{2} \wedge d \bar{z}^{2} \wedge d z^{3} \wedge d \bar{z}^{3}-c_{2} d z^{1} \wedge d \bar{z}^{1} \wedge d z^{3} \wedge d \bar{z}^{3}-c_{5} d z^{1} \wedge d \bar{z}^{1} \wedge d z^{2} \wedge d \bar{z}^{2} \\
& +\frac{1}{2}\left(c_{4}-i c_{3}\right) d z^{1} \wedge d \bar{z}^{2} \wedge d z^{3} \wedge d \bar{z}^{3}-\frac{1}{2}\left(c_{4}+i c_{3}\right) d \bar{z}^{1} \wedge d z^{2} \wedge d z^{3} \wedge d \bar{z}^{3}
\end{aligned}
$$

Thus, we are lead to define the following Kähler moduli in type IIB :

$$
\begin{aligned}
& \mathcal{T}^{1}=g_{11}+i c_{1} \quad, \quad \mathcal{T}^{2}=g_{22}+i c_{2} \quad, \quad \mathcal{T}^{5}=\operatorname{Re}\left(\mathcal{T}^{5}\right)+i c_{5}, \\
& \mathcal{T}^{3}=g_{13}+i c_{4} \quad, \quad \mathcal{T}^{4}=g_{14}+i c_{3} .
\end{aligned}
$$

The Kähler potential for the Kähler moduli $\mathcal{T}^{j}$ may be easily obtained from the volume form:

$$
\begin{aligned}
K_{\mathcal{K}} & =-\ln \operatorname{Vol}\left(T^{6} / \mathbf{Z}_{4}\right)=-\ln \frac{1}{6} \int_{T^{6}} J \wedge J \wedge J+\ln 4 \\
& =-\ln \frac{1}{8}\left\{\left(\mathcal{T}^{5}+\overline{\mathcal{T}}^{5}\right)\left[\left(\mathcal{T}^{1}+\overline{\mathcal{T}}^{1}\right)\left(\mathcal{T}^{2}+\overline{\mathcal{T}}^{2}\right)-\left(\mathcal{T}^{3}+\overline{\mathcal{T}}^{3}\right)^{2}-\left(\mathcal{T}^{4}+\overline{\mathcal{T}}^{4}\right)^{2}\right]\right\}+\ln 4
\end{aligned}
$$

Note, that $\operatorname{Vol}\left(T^{6} / \mathbf{Z}_{4}\right)=1 / 4 \operatorname{Vol}\left(T^{6}\right)$. Besides, the piece in the bracket $\frac{1}{4}[\ldots]$ is the volume $\sqrt{\operatorname{det} g}=g_{11} g_{22}-g_{13}^{2}-g_{14}^{2}$ of the 4 -torus $T^{4}$. The holomorphic field-redefinition

$$
\tilde{\mathcal{T}}^{3}=\mathcal{T}^{3}+i \mathcal{T}^{4}=g_{13}+i g_{14}+i c_{4}-c_{3} \quad, \quad \widetilde{\mathcal{T}}^{4}=\mathcal{T}^{3}-i \mathcal{T}^{4}=g_{13}-i g_{14}+i c_{4}+c_{3}
$$


would lead to the Kähler potential:

$$
\begin{aligned}
\widetilde{K}_{\mathcal{K}} & =-\ln \left\{\left(\mathcal{T}^{5}+\overline{\mathcal{T}}^{5}\right)\left[\left(\mathcal{T}^{1}+\overline{\mathcal{T}}^{1}\right)\left(\mathcal{T}^{2}+\overline{\mathcal{T}}^{2}\right)-\left(\widetilde{\mathcal{T}}^{3}+\overline{\widetilde{\mathcal{T}}}^{4}\right)\left(\overline{\widetilde{\mathcal{T}}}^{3}+\widetilde{\mathcal{T}}^{4}\right)\right]\right\} \\
& =-\ln \left(\mathcal{T}^{5}+\overline{\mathcal{T}}^{5}\right)-\ln \operatorname{det}\left(\mathcal{T}+\mathcal{T}^{\dagger}\right) \quad, \quad \mathcal{T}=\left(\begin{array}{cc}
\mathcal{T}^{1} & \widetilde{\mathcal{T}}^{3} \\
\widetilde{\mathcal{T}}^{4} & \mathcal{T}^{2}
\end{array}\right) .
\end{aligned}
$$

This form of the Kähler potential reproduces the formula given in (2.7) with the moduli fields $\mathcal{T}^{i}$ parameterized by the underlying string background. The two different forms (2.41) and (2.43) simply reflect the isomorphy $\frac{S U(2,2)}{S U(2) \times S U(2) \times U(1)} \simeq \frac{S O(4,2)}{S O(4) \times S O(2)}$. Finally, with the relation (2.8), we obtain the correct relations between the string-theoretical moduli fields $\mathcal{T}^{i}$ and the field-theoretical fields $T^{i}$. With

$$
\operatorname{Vol}\left(X_{6}\right)=\frac{1}{32}\left\{\left(\mathcal{T}^{5}+\overline{\mathcal{T}}^{5}\right)\left[\left(\mathcal{T}^{1}+\overline{\mathcal{T}}^{1}\right)\left(\mathcal{T}^{2}+\overline{\mathcal{T}}^{2}\right)-\left(\mathcal{T}^{3}+\overline{\mathcal{T}}^{3}\right)^{2}-\left(\mathcal{T}^{4}+\overline{\mathcal{T}}^{4}\right)^{2}\right]\right\},
$$

we obtain the following field-theoretical moduli fields:

$$
\begin{aligned}
& T^{1}=\frac{1}{32}\left(\mathcal{T}^{2}+\overline{\mathcal{T}}^{2}\right)\left(\mathcal{T}^{5}+\overline{\mathcal{T}}^{5}\right)+i c_{1} \quad, \quad T^{2}=\frac{1}{32}\left(\mathcal{T}^{1}+\overline{\mathcal{T}}^{1}\right)\left(\mathcal{T}^{5}+\overline{\mathcal{T}}^{5}\right)+i c_{2}, \\
& T^{3}=-\frac{1}{16}\left(\mathcal{T}^{3}+\overline{\mathcal{T}}^{3}\right)\left(\mathcal{T}^{5}+\overline{\mathcal{T}}^{5}\right)+i c_{3} \quad, \quad T^{4}=-\frac{1}{16}\left(\mathcal{T}^{4}+\overline{\mathcal{T}}^{4}\right)\left(\mathcal{T}^{5}+\overline{\mathcal{T}}^{5}\right)+i c_{4}, \\
& T^{5}=\frac{1}{32}\left[\left(\mathcal{T}^{1}+\overline{\mathcal{T}}^{1}\right)\left(\mathcal{T}^{2}+\overline{\mathcal{T}}^{2}\right)-\left(\mathcal{T}^{3}+\overline{\mathcal{T}}^{3}\right)^{2}-\left(\mathcal{T}^{4}+\overline{\mathcal{T}}^{4}\right)^{2}\right]+i c_{5}
\end{aligned}
$$

The Kähler potential (2.5), written in terms of these fields, becomes:

$$
\begin{aligned}
\kappa_{4}^{-2} K & =-\ln (S+\bar{S})+K_{C S}-\ln 4 \\
& -\ln \left\{\left(T^{5}+\bar{T}^{5}\right)\left[\left(T^{1}+\bar{T}^{1}\right)\left(T^{2}+\bar{T}^{2}\right)-\frac{1}{4}\left(T^{3}+\bar{T}^{3}\right)^{2}-\frac{1}{4}\left(T^{4}+\bar{T}^{4}\right)^{2}\right]\right\} .
\end{aligned}
$$

$\underline{\text { Kähler moduli space } \mathcal{M}_{\mathcal{K}}=\frac{S U(3,3)}{S U(3) \times S U(3) \times U(1)}}$

The simplest realization of this moduli space $\mathcal{M}_{\mathcal{K}}$ appears in the $\mathbf{Z}_{3}$-orbifold with the block-diagonal orbifold action:

$Q: e_{1} \longrightarrow e_{2}, e_{2} \longrightarrow-e_{1}-e_{2} \quad, e_{3} \longrightarrow e_{4}, e_{4} \longrightarrow-e_{3}-e_{4}, e_{5} \longrightarrow e_{6}, e_{6} \longrightarrow-e_{5}-e_{6}$.

on the six roots $\left\{e_{i}\right\}$ describing the six-torus $T^{6}$. According to (2.12) this twist allows for the metric background

$$
g=\left(\begin{array}{cccccc}
g_{11} & -\frac{1}{2} g_{11} & g_{13} & g_{14} & g_{15} & g_{16} \\
-\frac{1}{2} g_{11} & g_{11} & -g_{13}-g_{14} & g_{13} & -g_{15}-g_{16} & g_{15} \\
g_{13} & -g_{13}-g_{14} & g_{33} & -\frac{1}{2} g_{33} & g_{35} & g_{36} \\
g_{14} & g_{13} & -\frac{1}{2} g_{33} & g_{33} & -g_{35}-g_{36} & g_{35} \\
g_{15} & -g_{15}-g_{16} & g_{35} & -g_{35}-g_{36} & g_{55} & -\frac{1}{2} g_{55} \\
g_{16} & g_{15} & g_{36} & g_{35} & -\frac{1}{2} g_{55} & g_{55}
\end{array}\right)
$$


characterized by nine metric parameters. Following (2.14) we introduce complex structures and the complex coordinates $z^{1}, z^{2}, z^{3}$

$$
d z^{1}=\frac{1}{\sqrt{3}}\left(d x^{1}+\rho d y^{1}\right), \quad d z^{2}=\frac{1}{\sqrt{3}}\left(d x^{2}+\rho d y^{2}\right), \quad d z^{3}=\frac{1}{\sqrt{3}}\left(d x^{3}+\rho d y^{3}\right) .
$$

W.r.t. them, the twist (2.46) acts diagonally, i.e. $\Theta: z^{1} \rightarrow \rho z^{1}, z^{2} \rightarrow \rho z^{2}, z^{3} \rightarrow \rho z^{3}$, with $\rho=e^{\frac{2 \pi i}{3}}$. W.r.t. these coordinates the Kähler form $J$ becomes:

$$
\begin{aligned}
-i J & =\frac{3}{2} g_{11} d z^{1} \wedge d \bar{z}^{1}+\frac{3}{2} g_{33} d z^{2} \wedge d \bar{z}^{2}+\frac{3}{2} g_{55} d z^{3} \wedge d \bar{z}^{3} \\
& -\sqrt{3} i\left(\rho g_{13}-g_{14}\right) d z^{1} \wedge d \bar{z}^{2}-\sqrt{3} i\left(\rho g_{15}-g_{16}\right) d z^{1} \wedge d \bar{z}^{3} \\
& -\sqrt{3} i\left(\rho g_{35}-g_{36}\right) d z^{2} \wedge d \bar{z}^{3}+\sqrt{3} i\left(\bar{\rho} g_{13}-g_{14}\right) d z^{2} \wedge d \bar{z}^{1} \\
& +\sqrt{3} i\left(\bar{\rho} g_{15}-g_{16}\right) d z^{3} \wedge d \bar{z}^{1}+\sqrt{3} i\left(\bar{\rho} g_{35}-g_{36}\right) d z^{3} \wedge d \bar{z}^{2}
\end{aligned}
$$

Expressed w.r.t. the integer cohomology $H^{2}\left(X_{6}, \mathbf{Z}\right)$ we obtain:

$$
\begin{aligned}
J & =\frac{\sqrt{3}}{2} g_{11} d x^{1} \wedge d y^{1}+\frac{\sqrt{3}}{2} g_{33} d x^{2} \wedge d y^{2}+\frac{\sqrt{3}}{2} g_{55} d x^{3} \wedge d y^{3} \\
& -\frac{1}{\sqrt{3}}\left[\left(g_{13}+2 g_{14}\right)\left(d x^{1} \wedge d x^{2}-d y^{1} \wedge d x^{2}+d y^{1} \wedge d y^{2}\right)-\left(2 g_{13}+g_{14}\right)\left(d x^{1} \wedge d y^{2}-d y^{1} \wedge d x^{2}\right)\right] \\
& -\frac{1}{\sqrt{3}}\left[\left(g_{15}+2 g_{16}\right)\left(d x^{1} \wedge d x^{3}-d y^{1} \wedge d x^{3}+d y^{1} \wedge d y^{3}\right)-\left(2 g_{15}+g_{16}\right)\left(d x^{1} \wedge d y^{3}-d y^{1} \wedge d x^{3}\right)\right] \\
& -\frac{1}{\sqrt{3}}\left[\left(g_{35}+2 g_{36}\right)\left(d x^{2} \wedge d x^{3}-d y^{2} \wedge d x^{3}+d y^{2} \wedge d y^{3}\right)-\left(2 g_{35}+g_{36}\right)\left(d x^{2} \wedge d y^{3}-d y^{2} \wedge d x^{3}\right)\right] .
\end{aligned}
$$

Obviously, the 2-forms in the bracket represent the nine twist-invariant two-forms of the $\mathbf{Z}_{3}$-orbifold. The Ramond 4 -form $C_{4}$ has nine independent parameters and may be expanded w.r.t. a twist-invariant basis of $H^{4}\left(X_{6}, \mathbf{Z}\right)$ :

$$
\begin{aligned}
C_{4} & =c_{1} d x^{2} \wedge d y^{2} \wedge d x^{3} \wedge d y^{3}+c_{2} d x^{1} \wedge d y^{1} \wedge d x^{3} \wedge d y^{3}+c_{3} d x^{1} \wedge d y^{1} \wedge d x^{2} \wedge d y^{2} \\
& +\frac{1}{3}\left(c_{5}-2 c_{4}\right)\left(d x^{1} \wedge d x^{2}-d y^{1} \wedge d x^{2}+d y^{1} \wedge d y^{2}\right) \wedge d x^{3} \wedge d y^{3} \\
& +\frac{1}{3}\left(c_{4}-2 c_{5}\right)\left(d x^{1} \wedge d y^{2}-d y^{1} \wedge d x^{2}\right) \wedge d x^{3} \wedge d y^{3} \\
& +\frac{1}{3}\left(c_{7}-2 c_{6}\right)\left(d x^{1} \wedge d x^{3}-d y^{1} \wedge d x^{3}+d y^{1} \wedge d y^{3}\right) \wedge d x^{2} \wedge d y^{2} \\
& +\frac{1}{3}\left(c_{6}-2 c_{7}\right)\left(d x^{1} \wedge d y^{3}-d y^{1} \wedge d x^{3}\right) \wedge d x^{2} \wedge d y^{2} \\
& +\frac{1}{3}\left(c_{9}-2 c_{8}\right)\left(d x^{2} \wedge d x^{3}-d y^{2} \wedge d x^{3}+d y^{2} \wedge d y^{3}\right) \wedge d x^{1} \wedge d y^{1} \\
& +\frac{1}{3}\left(c_{8}-2 c_{9}\right)\left(d x^{2} \wedge d y^{3}-d y^{2} \wedge d x^{3}\right) \wedge d x^{1} \wedge d y^{1}
\end{aligned}
$$


This expansion has the property that the basis w.r.t. which the 4 -form $C_{4}$ is expanded, is Hodge-dual to the twist-invariant basis of 2-forms appearing in the expansion of $B_{2}$, given in Eq. (D.5) of appendix E. W.r.t. $H^{4}\left(X_{6}, \mathbf{C}\right)$, the expansion (2.51) becomes:

$$
\begin{aligned}
C_{4} & =-3 c_{1} d z^{2} \wedge d \bar{z}^{2} \wedge d z^{3} \wedge d \bar{z}^{3}-3 c_{2} d z^{1} \wedge d \bar{z}^{1} \wedge d z^{3} \wedge d \bar{z}^{3}-3 c_{3} d z^{1} \wedge d \bar{z}^{1} \wedge d z^{2} \wedge d \bar{z}^{2} \\
& -i \sqrt{3}\left(c_{4}+\rho c_{5}\right) d z^{1} \wedge d \bar{z}^{2} \wedge d z^{3} \wedge d \bar{z}^{3}-i \sqrt{3}\left(c_{4}+\bar{\rho} c_{5}\right) d \bar{z}^{1} \wedge d z^{2} \wedge d z^{3} \wedge d \bar{z}^{3} \\
& -i \sqrt{3}\left(c_{6}+\rho c_{7}\right) d z^{1} \wedge d \bar{z}^{3} \wedge d z^{2} \wedge d \bar{z}^{2}-i \sqrt{3}\left(c_{6}+\bar{\rho} c_{7}\right) d \bar{z}^{1} \wedge d z^{3} \wedge d z^{2} \wedge d \bar{z}^{2} \\
& -i \sqrt{3}\left(c_{8}+\rho c_{9}\right) d z^{2} \wedge d \bar{z}^{3} \wedge d z^{1} \wedge d \bar{z}^{1}-i \sqrt{3}\left(c_{8}+\bar{\rho} c_{9}\right) d \bar{z}^{2} \wedge d z^{3} \wedge d z^{1} \wedge d \bar{z}^{1} .
\end{aligned}
$$

In the type $I I B$ orientifold we are discussing, the metric components of $J$ are complexified with the respective components of $C_{4}$ and lead to:

$$
\begin{array}{rlrl}
\mathcal{T}^{1} & =\frac{\sqrt{3}}{2} g_{11}+i c_{1} \quad, \quad \mathcal{T}^{2}=\frac{\sqrt{3}}{2} g_{33}+i c_{2} \quad, \quad \mathcal{T}^{3}=\frac{\sqrt{3}}{2} g_{55}+i c_{3}, \\
\mathcal{T}^{4} & =-\frac{1}{\sqrt{3}}\left(g_{13}+2 g_{14}\right)+i c_{4} \quad, \quad \mathcal{T}^{5}=\frac{1}{\sqrt{3}}\left(2 g_{13}+g_{14}\right)+i c_{5}, \\
\mathcal{T}^{6} & =-\frac{1}{\sqrt{3}}\left(g_{15}+2 g_{16}\right)+i c_{6} \quad, \quad \mathcal{T}^{7}=\frac{1}{\sqrt{3}}\left(2 g_{15}+g_{16}\right)+i c_{7}, \\
\mathcal{T}^{8} & =-\frac{1}{\sqrt{3}}\left(g_{35}+2 g_{36}\right)+i c_{8} \quad, & \mathcal{T}^{9} & =\frac{1}{\sqrt{3}}\left(2 g_{35}+g_{36}\right)+i c_{9} .
\end{array}
$$

The Kähler potential for the Kähler moduli $\mathcal{T}^{j}$ may be easily obtained from the volume form:

$$
K_{\mathcal{K}}=-\ln \frac{1}{6} \int_{X_{6}} J \wedge J \wedge J=-\ln \operatorname{Vol}\left(X_{6}\right),
$$

with

$$
\operatorname{Vol}\left(T^{6} / \mathbf{Z}_{3}\right)=\frac{1}{3} \cdot \frac{1}{6} \mathcal{K}_{i j k} \operatorname{Re}\left(\mathcal{T}^{i}\right) \operatorname{Re}\left(\mathcal{T}^{j}\right) \operatorname{Re}\left(\mathcal{T}^{k}\right)=\frac{1}{3} \sqrt{\operatorname{det}(g)},
$$

and the triple intersection numbers $\mathcal{K}_{i j k}$ :

$$
\begin{aligned}
& \mathcal{K}_{123}=1, \mathcal{K}_{344}=-2, \mathcal{K}_{345}=-1, \mathcal{K}_{355}=-2, \mathcal{K}_{266}=-2, \\
& \mathcal{K}_{267}=-1, \mathcal{K}_{277}=-2, \mathcal{K}_{468}=1, \mathcal{K}_{568}=2, \mathcal{K}_{478}=-1, \\
& \mathcal{K}_{578}=1, \mathcal{K}_{188}=-2, \mathcal{K}_{469}=2, \mathcal{K}_{569}=1, \mathcal{K}_{479}=1, \\
& \mathcal{K}_{579}=2, \mathcal{K}_{189}=-1, \mathcal{K}_{199}=-2 .
\end{aligned}
$$

Again, through a holmorphic field-redefinition we may pass to the moduli fields :

$$
\begin{aligned}
& \widetilde{\mathcal{T}}^{4}=-i\left(\rho g_{13}-g_{14}\right)+\frac{i}{\sqrt{3}}\left(c_{4}+\rho c_{5}\right), \tilde{\mathcal{T}}^{7}=i\left(\bar{\rho} g_{13}-g_{14}\right)+\frac{i}{\sqrt{3}}\left(c_{4}+\bar{\rho} c_{5}\right), \\
& \tilde{\mathcal{T}}^{5}=-i\left(\rho g_{15}-g_{16}\right)+\frac{i}{\sqrt{3}}\left(c_{6}+\rho c_{7}\right), \tilde{\mathcal{T}}^{8}=i\left(\bar{\rho} g_{15}-g_{16}\right)+\frac{i}{\sqrt{3}}\left(c_{6}+\bar{\rho} c_{7}\right), \\
& \widetilde{\mathcal{T}}^{6}=-i\left(\rho g_{35}-g_{36}\right)+\frac{i}{\sqrt{3}}\left(c_{8}+\rho c_{9}\right), \tilde{\mathcal{T}}^{9}=i\left(\bar{\rho} g_{35}-g_{36}\right)+\frac{i}{\sqrt{3}}\left(c_{8}+\bar{\rho} c_{9}\right) .
\end{aligned}
$$


In terms of these moduli, the Kähler potential becomes:

$$
\widetilde{K}_{\mathcal{K}}=-\ln \operatorname{det}\left(\mathcal{T}+\mathcal{T}^{\dagger}\right) \quad, \quad \mathcal{T}=\left(\begin{array}{ccc}
\mathcal{T}^{1} & \widetilde{\mathcal{T}}^{4} & \widetilde{\mathcal{T}}^{5} \\
\widetilde{\mathcal{T}}^{7} & \mathcal{T}^{2} & \widetilde{\mathcal{T}}^{6} \\
\widetilde{\mathcal{T}}^{8} & \widetilde{\mathcal{T}}^{9} & \mathcal{T}^{3}
\end{array}\right)
$$

in agreement with the expression given in (2.7). Finally, from (2.8) and (2.55) we may deduce the nine holomorphic moduli fields $T^{i}$

$$
T^{i}=\frac{1}{2} \mathcal{K}_{i j k} \operatorname{Re}\left(\mathcal{T}^{j}\right) \operatorname{Re}\left(\mathcal{T}^{k}\right)+i c_{i}
$$

with the intersection numbers given in (2.56). The full Kähler potential (2.5), written in terms of the fields (2.59), becomes

$$
\kappa_{4}^{-2} K=-\ln (S+\bar{S})-\ln \left[\frac{1}{8} \widetilde{\mathcal{K}}_{i j k}\left(T^{i}+\bar{T}^{i}\right)\left(T^{j}+\bar{T}^{j}\right)\left(T^{k}+\bar{T}^{k}\right)\right],
$$

with:

$$
\begin{array}{lllll}
\widetilde{\mathcal{K}}_{123}=\frac{1}{2}, & \widetilde{\mathcal{K}}_{344}=-\frac{1}{3}, & \widetilde{\mathcal{K}}_{345}=\frac{1}{6}, & \widetilde{\mathcal{K}}_{355}=-\frac{1}{3}, & \widetilde{\mathcal{K}}_{266}=-\frac{1}{3}, \\
\widetilde{\mathcal{K}}_{267}=\frac{1}{6}, & \widetilde{\mathcal{K}}_{277}=-\frac{1}{3}, & \widetilde{\mathcal{K}}_{568}=\frac{1}{6}, & \widetilde{\mathcal{K}}_{478}=-\frac{1}{6}, & \widetilde{\mathcal{K}}_{188}=-\frac{1}{3}, \\
\widetilde{\mathcal{K}}_{469}=\frac{1}{6} & \widetilde{\mathcal{K}}_{569}=-\frac{1}{6}, & \widetilde{\mathcal{K}}_{579}=\frac{1}{6}, & \widetilde{\mathcal{K}}_{189}=\frac{1}{6}, & \widetilde{\mathcal{K}}_{199}=-\frac{1}{3} .
\end{array}
$$

\subsection{String-theoretical Kähler moduli $\mathcal{T}^{i}$ vs. field-theoretical fields $T^{i}$}

The imaginary part of the modulus $\mathcal{T}^{i}$, defined in $E q$. (2.15), follows from the integral $\operatorname{Im}\left(\mathcal{T}^{i}\right)=\int_{C^{i}} C_{4}$ of the Ramond 4-form over a certain 4-cycle $C^{i}$. A D7-brane has the world-volume Chern-Simons coupling $\int C_{4} \wedge F \wedge F$. Hence, a $D 7$-brane wrapped around this 4 -cycle $C_{i}$ gives rise to the $C P$-odd gauge term $\int \operatorname{Im}\left(\mathcal{T}^{i}\right) F \wedge F$ in $D=4$. On the other hand, the real parts of the moduli $\mathcal{T}^{i}$, which derive from the underlying string background ( $c f$. Subsection 2.3) do not yet properly fit into complex scalars $T^{i}$ of $\mathrm{N}=1$ chiral multiplets in field theory. According to the previous discussion, the real part $\operatorname{Re}\left(T^{i}\right)$ of those scalar fields has to describe the gauge coupling of a $D 7$-brane, which is wrapped around the four-cycle $C_{i}$. This coupling is measured by the volume of this 4 -cycle $C_{i}$. More precisely, from the Born-Infeld action $e^{-\phi_{10}} \int d^{8} \xi \operatorname{det}\left(g+2 \pi \alpha^{\prime} F\right)^{1 / 2}$ we derive the $C P$-even gauge-coupling $\operatorname{Re}\left(T^{i}\right):=e^{-\phi_{10}} \int_{C_{i}} d^{4} \xi \operatorname{det}(g)^{1 / 2}$. In order for the $D 7$-brane to respect $1 / 2$ of the supersymmetry of the bulk theory, which is $\mathrm{N}=2$ in $D=4$, the internal 4 -cycle $C_{i}$ the $D 7$-brane is wrapped on has to fulfill the calibration condition [49]:

$$
e^{-\phi_{10}} \int_{C_{i}} d^{4} \xi \operatorname{det}(g)^{1 / 2}=\frac{1}{2} \int_{C_{i}} J \wedge J
$$


Note, that the r.h.s. just describes the volume of the 4 -cycle $C_{i}$. Hence, the real part of the correct holomorphic modulus $T^{i}$ is:

$$
\operatorname{Re}\left(T^{i}\right):=e^{-\phi_{10}} \int_{C_{i}} d^{4} \xi \operatorname{det}(g)^{1 / 2}=\frac{1}{2} \int_{C_{i}} J \wedge J
$$

More precisely, with $\omega_{i}$ the Poincaré dual 2-form of the $4-$ cycle $C_{i}$, we have: $\int_{C_{i}} J \wedge J=$ $\int_{X_{6}} \omega_{i} \wedge J \wedge J$. With $J=\sum_{j=1}^{h_{(1,1)}} \operatorname{Re}\left(\mathcal{T}^{j}\right) \omega_{j}$ we may write

$$
\operatorname{Re}\left(T^{i}\right):=\frac{1}{6} \frac{\partial}{\partial \operatorname{Re}\left(\mathcal{T}^{i}\right)} \int_{X_{6}} J \wedge J \wedge J=\frac{\partial}{\partial \operatorname{Re}\left(\mathcal{T}^{i}\right)} \operatorname{Vol}\left(X_{6}\right)
$$

which proves (2.8). Furthermore, with $\int_{X_{6}} J \wedge J \wedge J=\mathcal{K}_{i j k} \operatorname{Re}\left(\mathcal{T}^{i}\right) \operatorname{Re}\left(\mathcal{T}^{j}\right) \operatorname{Re}\left(\mathcal{T}^{k}\right)$, and $\mathcal{K}_{i j k}$ the intersection form of the $\mathrm{CYM} X_{6}$, we may also write

$$
\operatorname{Re}\left(T^{i}\right):=\frac{1}{2} \mathcal{K}_{i j k} \operatorname{Re}\left(\mathcal{T}^{j}\right) \operatorname{Re}\left(\mathcal{T}^{k}\right)
$$

which gives the volume of the 4 -cycle (in the string frame).

For the case when the Kähler moduli space is described by the coset space $\left(\frac{S U(1,1)}{U(1)}\right)^{3}$ we have worked out the field-theoretical moduli fields $T^{i}$ in $E q$. (2.31), for the coset $\frac{S U(2,2)}{S U(2) \times S U(2) \times U(1)} \times \frac{S U(1,1)}{U(1)}$, in $E q$. (2.44), and for the coset $\frac{S U(3,3)}{S U(3) \times S U(3) \times U(1)}$ in $E q$. (2.59).

\subsection{Three-form flux $G_{3}$ in $\mathbf{Z}_{N}$ and $\mathbf{Z}_{N} \times \mathbf{Z}_{M}$-orbifolds}

Let us now give non-vanishing vevs to some of the (untwisted) flux components $H_{i j k}$ and $F_{i j k}$, with $F_{3}=d C_{2}, H_{3}=d B_{2}$. The two 3 -forms $F_{3}, H_{3}$ are organized in the $S L(2, \mathbf{Z})_{S}$ covariant field:

$$
G_{3}=F_{3}+i S H_{3}
$$

On the torus $T^{6}$, we would have $20+20$ independent internal components for $H_{i j k}$ and $F_{i j k}$. However, only a portion of them is invariant under the orbifold group $\Gamma$. More precisely, of the 20 complex (untwisted) components comprising the flux $G_{3}$, only $2 h_{(2,1)}^{\text {untw. }}\left(X_{6}\right)+2$ survive the orbifold twist. The orientifold action $\Omega(-1)^{F_{L}} I_{6}$ producing $O 3-$ planes does not give rise to any further restrictions. If the orbifold group $\Gamma$ contains $\mathbf{Z}_{2}$-elements $\theta$ which leave the $j$-th complex plane fixed, we also encounter $O 7_{j}$-planes transverse to the $j$-th plane. Since $I_{2}^{j}=I_{6} \theta$, the orientifold generator $\Omega(-1)^{F_{L}} I_{2}^{j}$ does not put further restrictions on the $2 h_{(2,1)}^{\text {untw. }}\left(X_{6}\right)+2$ twist invariant components. Hence, the allowed flux components are most conveniently found in the complex basis, in which the orbifold group $\Gamma$ acts diagonally. In the following, we shall concentrate on the orientifold/orbifolds $T^{6} /\left(\Gamma+\Gamma \Omega I_{6}\right)$, with $\Gamma$ 
being one of the orbifold twists $\mathbf{Z}_{N}$ or $\mathbf{Z}_{N} \times \mathbf{Z}_{M}$ encountered above. Note that $O 7$-planes appear in the case that the orbifold twist $\Gamma$ is of even order.

The most general 3 -form flux $G_{3}$ on $T^{6}$ has 20 components, which appear in the expansion

$$
\frac{1}{(2 \pi)^{2} \alpha^{\prime}} G_{3}=\sum_{i=0}^{3}\left(A^{i} \omega_{A_{i}}+B^{i} \omega_{B_{i}}\right)+\sum_{j=1}^{6}\left(C^{j} \omega_{C_{j}}+D^{j} \omega_{D_{j}}\right)
$$

w.r.t. the complex 3-form cohomology $H^{3}=H^{(3,0)} \oplus H^{(2,1)} \oplus H^{(1,2)} \oplus H^{(0,3)}$ :

$$
\begin{array}{ll}
\omega_{A_{0}}=d z^{1} \wedge d z^{2} \wedge d z^{3} & , \quad \omega_{B_{0}}=d \bar{z}^{1} \wedge d \bar{z}^{2} \wedge d \bar{z}^{3} \\
\omega_{A_{1}}=d \bar{z}^{1} \wedge d z^{2} \wedge d z^{3} & , \quad \omega_{B_{1}}=d z^{1} \wedge d \bar{z}^{2} \wedge d \bar{z}^{3} \\
\omega_{A_{2}}=d z^{1} \wedge d \bar{z}^{2} \wedge d z^{3} & , \quad \omega_{B_{2}}=d \bar{z}^{1} \wedge d z^{2} \wedge d \bar{z}^{3} \\
\omega_{A_{3}}=d z^{1} \wedge d z^{2} \wedge d \bar{z}^{3} & , \quad \omega_{B_{3}}=d \bar{z}^{1} \wedge d \bar{z}^{2} \wedge d z^{3} \\
\omega_{C_{1}}=d z^{1} \wedge d \bar{z}^{1} \wedge d z^{2} & , \quad \omega_{D_{1}}=d z^{1} \wedge d \bar{z}^{1} \wedge d \bar{z}^{2} \\
\omega_{C_{2}}=d z^{1} \wedge d \bar{z}^{1} \wedge d z^{3} \quad, \quad \omega_{D_{2}}=d z^{1} \wedge d \bar{z}^{1} \wedge d \bar{z}^{3} \\
\omega_{C_{3}}=d z^{1} \wedge d z^{2} \wedge d \bar{z}^{2} \quad, \quad \omega_{D_{3}}=d \bar{z}^{1} \wedge d z^{2} \wedge d \bar{z}^{2} \\
\omega_{C_{4}}=d z^{2} \wedge d \bar{z}^{2} \wedge d z^{3} \quad, \quad \omega_{D_{4}}=d z^{2} \wedge d \bar{z}^{2} \wedge d \bar{z}^{3} \\
\omega_{C_{5}}=d z^{1} \wedge d z^{3} \wedge d \bar{z}^{3} \quad, \quad \omega_{D_{5}}=d \bar{z}^{1} \wedge d z^{3} \wedge d \bar{z}^{3} \\
\omega_{C_{6}}=d z^{2} \wedge d z^{3} \wedge d \bar{z}^{3} \quad, \quad \omega_{D_{6}}=d \bar{z}^{2} \wedge d z^{3} \wedge d \bar{z}^{3} .
\end{array}
$$

The $\omega_{A_{i}}, \omega_{B_{i}}$ correspond to flux components with all one-forms coming from different planes, while the $\omega_{C_{i}}, \omega_{D_{i}}$ are flux components with two one-forms coming from the same plane. The latter we have just written down for completeness, as they are projected out in all orbifolds. In the $\mathbf{Z}_{2} \times \mathbf{Z}_{2}$ orientifold/orbifold, which allows for the largest number of (untwisted) fluxes [14], all $\omega_{A_{i}}$ and $\omega_{B_{i}}$ remain, whereas in most other orbifolds only $\omega_{A_{0}}$ and $\omega_{B_{0}}$ survive. In appendix B we list the 3-form flux components, which are invariant under the respective orbifold action $\Gamma$. That the $(0,3)$ and $(3,0)$-flux always survive is quite clear, as the $(3,0)$-flux corresponds to the Calabi-Yau 3-form $\Omega$, which is always present, and the (0,3)-flux to its conjugate.

While in the form (2.67), the cohomology structure of $G_{3}$ is manifest, in order to impose the flux quantization on $G_{3}$, i.e.

$$
\frac{1}{(2 \pi)^{2} \alpha^{\prime}} \int_{C_{3}} F_{3} \in n_{0} \mathbf{Z} \quad, \quad \frac{1}{(2 \pi)^{2} \alpha^{\prime}} \int_{C_{3}} H_{3} \in n_{0} \mathbf{Z}
$$


with some integer $n_{0}$ (depending on the orbifold group $\Gamma$ ) to be specified later, one has to transform the forms (2.68) into a real basis of the following 20 elements

$$
\begin{aligned}
& \alpha_{0}=d x^{1} \wedge d x^{2} \wedge d x^{3} \quad, \quad \beta^{0}=d y^{1} \wedge d y^{2} \wedge d y^{3}, \\
& \alpha_{1}=d y^{1} \wedge d x^{2} \wedge d x^{3} \quad, \quad \beta^{1}=-d x^{1} \wedge d y^{2} \wedge d y^{3}, \\
& \alpha_{2}=d x^{1} \wedge d y^{2} \wedge d x^{3} \quad, \quad \beta^{2}=-d y^{1} \wedge d x^{2} \wedge d y^{3}, \\
& \alpha_{3}=d x^{1} \wedge d x^{2} \wedge d y^{3} \quad, \quad \beta^{3}=-d y^{1} \wedge d y^{2} \wedge d x^{3}, \\
& \gamma_{1}=d x^{1} \wedge d y^{1} \wedge d x^{2} \quad, \quad \delta^{1}=-d y^{2} \wedge d x^{3} \wedge d y^{3} \\
& \gamma_{2}=d x^{1} \wedge d y^{1} \wedge d x^{3} \quad, \quad \delta^{2}=-d x^{2} \wedge d y^{2} \wedge d y^{3}, \\
& \gamma_{3}=d x^{1} \wedge d x^{2} \wedge d y^{2} \quad, \quad \delta^{3}=-d y^{1} \wedge d x^{3} \wedge d y^{3}, \\
& \gamma_{4}=d x^{2} \wedge d y^{2} \wedge d x^{3} \quad, \quad \delta^{4}=-d x^{1} \wedge d y^{1} \wedge d y^{3} \\
& \gamma_{5}=d x^{1} \wedge d x^{3} \wedge d y^{3} \quad, \quad \delta^{5}=-d y^{1} \wedge d x^{2} \wedge d y^{2}, \\
& \gamma_{6}=d x^{2} \wedge d x^{3} \wedge d y^{3} \quad, \quad \delta^{6}=-d x^{1} \wedge d y^{1} \wedge d y^{2},
\end{aligned}
$$

with the six real periodic coordinates $x^{i}, y^{i}$ on the torus $T^{6}$, i.e. $x^{i} \cong x^{i}+1$ and $y^{i} \cong y^{i}+1$. The basis (2.70) has the property $\int_{X_{6}} \alpha_{i} \wedge \beta^{j}=\delta_{i}^{j}, \int_{X_{6}} \gamma_{i} \wedge \delta^{j}=\delta_{i}^{j}$, with the choice of orientation $\int_{X_{6}} d x^{1} \wedge d x^{2} \wedge d x^{3} \wedge d y^{1} \wedge d y^{2} \wedge d y^{3}=1$. In real notation, the flux has the form:

$$
\frac{1}{(2 \pi)^{2} \alpha^{\prime}} G_{3}=\sum_{i=0}^{3}\left[\left(a^{i}+i S c^{i}\right) \alpha_{i}+\left(b_{i}+i S d_{i}\right) \beta^{i}\right]+\sum_{j=1}^{6}\left[\left(e^{j}+i S g^{j}\right) \gamma_{j}+\left(f_{j}+i S h_{j}\right) \delta^{j}\right] .
$$

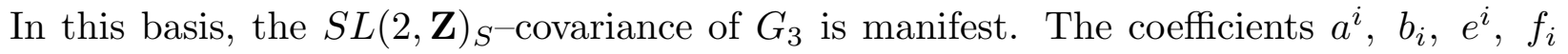
refer to the Ramond part of $G_{3}$, whereas the coefficients $c^{i}, d_{i}, g^{i}, h_{i}$ refer to the NeveuSchwarz part.

To pass from the complex basis (2.68) to the real basis (2.70), one introduces complex structures, i.e. the complex coordinates (cf. (2.14)):

$$
d z^{j}=\sum_{i=1}^{3} \rho_{i}^{j} d x^{i}+\tau_{i}^{j} d y^{i} \quad, \quad j=1,2,3
$$

Most of the parameters $\rho_{i}^{j}$ and $\tau_{i}^{j}$ are fixed through the orbifold twist $\Gamma$, with only those remaining undetermined, which correspond to the $\mathbf{Z}_{2}$-elements of $\Gamma$. The latter are eventually fixed through the flux quantization condition ( $c f$. appendix B). As we shall see in a moment, the specific values of the constants $\rho_{i}^{j}$ and $\tau_{i}^{j}$ are relevant for finding flux solutions.

Let us briefly comment on the integers $n_{0}$, introduced in the flux quantization conditions (2.69). It has been pointed out in Ref. [50], that there are subtleties for toroidal 
orientifolds due to additional 3-cycles, which are not present in the covering space $T^{6}$. If some integers are odd, additional discrete flux has to be turned on in order to meet the quantization rule for those 3-cycles. We may bypass these problems in the $\mathbf{Z}_{N}\left(\mathbf{Z}_{N} \times \mathbf{Z}_{M}\right)$ orientifolds, if we choose the quantization numbers to be multiples of $n_{0}=2 N\left(n_{0}=2 N M\right)$ and do not allow for discrete flux at the orientifold planes [51,52,53. Note, that for $h_{(2,1)}^{\text {twist. }} \neq 0$, in addition to the untwisted flux components $H_{i j k}$ and $F_{i j k}$ there may be also $N S N S$ - and $R R$-flux components from the twisted sector. We do not consider them here. It is assumed, that their quantization rules freeze the blowing up moduli at the orbifold singularities.

To illustrate the above procedure, we shall discuss the $\mathbf{Z}_{6-I I}$ orbifold with the lattice $(S U(2))^{2} \times S U(3) \times G_{2}$ and present the fluxes compatible with the complex structures of this orbifold. We will again present only one example, while the other orbifolds are treated in appendix B.

At this level, no supersymmetry conditions are imposed. Imposing further conditions will fix $S$ and the complex structure moduli (in case they are present in the particular orbifold) and/or constrain the coefficients $a_{i}, b_{i}, c_{i}, d_{i}$ which are real integers.

The $\mathbf{Z}_{6-I I}$ orbifold on the lattice $(S U(2))^{2} \times S U(3) \times G_{2}$ is a case with one complex structure modulus $\mathcal{U}^{3}$ left unfixed, therefore the flux takes the form 3

$$
\frac{1}{(2 \pi)^{2} \alpha^{\prime}} G_{3}=A_{0} \omega_{A_{0}}+A_{3} \omega_{A_{3}}+B_{0} \omega_{B_{0}}+B_{3} \omega_{B_{3}} \text {. }
$$

The $(3,0)$-form on this orbifold takes the form 1

$$
\begin{aligned}
\omega_{A_{0}}= & \frac{1}{3}\left\{3 \alpha_{0}+\sqrt{3} e^{5 \pi i / 6} \alpha_{1}+3 e^{2 \pi i / 3} \alpha_{2}\right. \\
& \left.+\mathcal{U}^{3}\left[3 \alpha_{3}-i\left(\sqrt{3} \beta_{0}+3 e^{\pi i / 6} \beta_{1}+\sqrt{3} e^{2 \pi i / 6} \beta_{2}\right)\right]+i \sqrt{3} \beta_{3}\right\}
\end{aligned}
$$

The one $(2,1)$-form surviving the twist takes the form

$$
\begin{aligned}
\omega_{A_{3}}= & \frac{1}{3}\left\{3 \alpha_{0}+\sqrt{3} e^{5 \pi i / 6} \alpha_{1}+3 e^{2 \pi i / 3} \alpha_{2}\right. \\
& \left.+\overline{\mathcal{U}}^{3}\left[3 \alpha_{3}-i\left(\sqrt{3} \beta_{0}+3 e^{\pi i / 6} \beta_{1}+\sqrt{3} e^{2 \pi i / 6} \beta_{2}\right)\right]+i \sqrt{3} \beta_{3}\right\} .
\end{aligned}
$$

3 In the remainder of this section and in the two appendices A, B the real and imaginary parts of the complex structure moduli $\mathcal{U}$ are exchanged compared to the previous setup.

4 Contrary to the normalization of $d z^{3}$ used in the context of the linear $\sigma$-model we do not use the prefactor $1 / \sqrt{\operatorname{Im} \mathcal{U}^{3}}$ here in order to obtain a holomorphic superpotential. 
$\omega_{B_{0}}$ and $\omega_{B_{3}}$ are the complex conjugates of the above. For the complex coefficients we find

$$
\begin{aligned}
A_{0}= & \frac{1}{2 \operatorname{Im} \mathcal{U}^{3}}\left\{e^{2 \pi i / 12} b_{0}-i b_{2}+i S\left(e^{2 \pi i / 12} d_{0}-i d_{2}\right)\right. \\
& \left.+\overline{\mathcal{U}}^{3}\left[\frac{1}{\sqrt{3}} e^{2 \pi i / 6} a_{0}+\frac{1}{\sqrt{3}} a_{2}+i S\left(-\sqrt{3} e^{2 \pi i / 6} c_{0}+\frac{1}{\sqrt{3}} c_{2}\right)\right]\right\} \\
B_{0}= & \frac{1}{2 \operatorname{Im} \mathcal{U}^{3}}\left\{e^{-2 \pi i / 12} b_{0}+i b_{2}+i S\left(e^{-2 \pi i / 12} d_{0}+i d_{2}\right)\right. \\
& \left.+\mathcal{U}^{3}\left[\frac{1}{\sqrt{3}} e^{-2 \pi i / 6} a_{0}+\frac{1}{\sqrt{3}} a_{2}+i S\left(\sqrt{3} e^{-2 \pi i / 6} c_{0}+\frac{1}{\sqrt{3}} c_{2}\right)\right]\right\} \\
A_{3}= & \frac{1}{2 \operatorname{Im} \mathcal{U}^{3}}\left\{e^{-10 \pi i / 12} b_{0}+i b_{2}+i S\left(e^{-10 \pi i / 12} d_{0}+i d_{2}\right)\right. \\
& \left.-\mathcal{U}^{3}\left[\frac{1}{\sqrt{3}} e^{2 \pi i / 6} a_{0}+\frac{1}{\sqrt{3}} a_{2}+i S\left(\sqrt{3} e^{2 \pi i / 6} c_{0}+\frac{1}{\sqrt{3}} c_{2}\right)\right]\right\} \\
B_{3}= & \frac{1}{2 \operatorname{Im} \mathcal{U}^{3}}\left\{e^{10 \pi i / 12} b_{0}-i b_{2}+i S\left(e^{10 \pi i / 12} d_{0}-i d_{2}\right)\right. \\
& \left.-\overline{\mathcal{U}}^{3}\left[\frac{1}{\sqrt{3}} e^{-2 \pi i / 6} a_{0}+\frac{1}{\sqrt{3}} a_{2}+i S\left(\sqrt{3} e^{-2 \pi i / 6} c_{0}+\frac{1}{\sqrt{3}} c_{2}\right)\right]\right\}
\end{aligned}
$$

Note that the normalization of the 3 -forms is $\int \omega_{A_{0}} \wedge \omega_{B_{0}}=2 i \operatorname{Im} \mathcal{U}^{3}$. Expressed in the real 3-forms, the flux takes the form

$$
\begin{aligned}
\frac{1}{(2 \pi)^{2} \alpha^{\prime}} G_{3}= & \left(a^{0}+i S c^{0}\right) \alpha_{0}+\frac{1}{3}\left(-a^{0}+a^{2}-i S\left(c^{0}-c^{2}\right)\right) \alpha_{1} \\
& +\left(a^{2}+i S c^{2}\right) \alpha_{2}+\left(-b_{0}+2 b_{2}+i S\left(-d_{0}+2 d_{2}\right)\right) \alpha_{3}+\left(b_{0}+i S d_{0}\right) \beta^{0} \\
& \left.+\left(b_{0}+b_{2}+i S\left(d_{0}+d_{2}\right)\right) \beta^{1}+\left(b_{2}+i S d_{2}\right) \beta^{2}+\frac{1}{3}\left(a^{0}+2 a^{2}+i S\left(c^{0}+2 c^{2}\right)\right) \beta^{3}\right] .
\end{aligned}
$$

\section{Vacuum structure of orientifolds in the orbifold limits}

In this section, we investigate the vacuum structure of type $I I B$ orientifold compactifications in their orbifold limits. The discussion is based on the following effective $\mathrm{N}=1$ superpotential

$$
W=W_{\text {flux }}\left(S, U^{j}\right)+W_{\mathrm{np}}\left(T^{i}\right),
$$

with:

$$
\begin{aligned}
W_{\text {flux }}\left(S, U^{j}\right) & =\frac{\lambda}{(2 \pi)^{2} \alpha^{\prime}} \int_{X_{6}} G_{3} \wedge \Omega, \\
W_{\mathrm{np}}\left(T^{i}\right) & =\sum_{i=1}^{h_{(1,1)}\left(X_{6}\right)} g^{i} e^{-h^{i} T^{i}} \quad, \quad g^{i} \in \mathbf{C}, h^{i} \in \mathbf{R}^{+} .
\end{aligned}
$$


The first term is the perturbative contribution to the superpotential due to non-vanishing 3-form fluxes [8], and it depends on the dilaton field $S$ and, if present, also on the untwisted complex structure moduli $U^{j}$ (with the normalization $\kappa_{10}^{-2}=\frac{\lambda}{(2 \pi)^{2} \alpha^{\prime}}$ ). The second term is of non-perturbative nature and depends on the untwisted Kähler moduli $T^{i}$. At this stage, we do not discuss any further the possible microscopic origin of the non-perturbative superpotential, but are only interested in a rather phenomenological way in its effect on the ground state structure of the orbifold models. Later in section 4, we shall provide some more details on the non-perturbative effects which may cause such a non-perturbative superpotential, namely wrapped Euclidean D3-branes and/or gaugino condensates. As a result, we will see that a non-perturbative superpotential of this type may emerge if also effects from blowing up modes of the orbifold singularities are taken into account. Hence this superpotential that depends only on the untwisted moduli $T^{i}$ can be regarded as a certain truncation of a more complete non-perturbative superpotential. This and several other aspects will be also the subject of a forthcoming publication [31.

The vacua of the effective $\mathrm{N}=1$ supergravity theory are determined by the associated scalar potential 54

$$
V=e^{\kappa_{4}^{2} K}\left(\left|D_{S} W\right|^{2}+\sum_{i=1}^{h_{(1,1)}\left(X_{6}\right)}\left|D_{T^{i}} W\right|^{2}+\sum_{j=1}^{h_{(2,1)}\left(X_{6}\right)}\left|D_{U^{j}} W\right|^{2}-3|W|^{2}\right)
$$

with the Kähler potential for the fields $S, T^{j}, U^{j}$. During the process of minimizing $V$, the following two aspects will become important: first, the supersymmetry conditions $D_{S, T^{i}, U^{j}} W=0$ will imply that generic supersymmetric AdS ground states are described by fluxes which are not anymore $I S D$ with only $G_{2,1}$ components, but rather will include $G_{0,3}$ and also all $I A S D$ (imaginary anti self-dual) types as well. The second issue concerns the stability of the obtained extrema after imposing the supersymmetry conditions. As stated already in the introduction, in the framework of the KKLT scenario, one has to require the absence of any tachyonic scalar fields, i.e. the (mass) ${ }^{2}$ of all scalars must be positive. This means that all eigenvalues of the scalar field mass matrix $\frac{\partial^{2} V}{\partial \phi_{\alpha} \partial \bar{\phi}_{\beta}}\left(\phi_{\alpha}, \phi_{\beta}=\right.$ $\left.S, U^{j}, T^{i}\right)$ must be positive. As we will see, this requirement can be only satisfied by those orbifolds which contain untwisted complex structure moduli $U^{j}$. In this way, we derive some severe constraints on which orbifolds can lead to stable vacua. This result is contrasted by the procedure originally applied in KKLT, where first the dilaton field $S$ and the complex structure moduli were integrated out by solving the flux supersymmetry conditions $D_{S} W_{\text {flux }}=D_{U^{j}} W_{\text {flux }}=0$ using $I S D(2,1)$ - or $(0,3)$-fluxes, and then plugging the obtained values for $S$ and $U^{j}$ back into $W$. This leads to a constant term $W_{0}$. However, the integrating-out procedure is in addition only consistent, if the masses of the integratedout fields $S$ and $U^{j}$ are heavy compared to the Kähler moduli $T^{i}$. Otherwise, the results on 
the vacuum structure and especially what concerns the stability problems are misleading and cannot be trusted anymore.

This problem has been emphasized and thoroughly discussed recently in Ref. 335. In this section, we want to generalize this discussion into several directions. First, we discuss under what conditions stable minima may be found if all moduli are minimized at once without first integrating out the complex structure moduli. This way, in subsection 3.5 we find a stable minimum for the case $h_{(1,1)}^{\text {untw. }}=3$ and $h_{(2,1)}^{\text {untw. }}=1$. On the other hand, in Ref. [35] it is has been proven that this case would not lead to a stable minimum, if the complex structure modulus was integrated out first. Secondly, in subsections 3.3 and 3.4, we shall investigate the KKLT scenario in toroidal orbifolds for more than one Kähler modulus and more general Kähler potentials ( $c f .(2.7)$ ) at fixed complex structure modulus. We find, that in those cases no stable minimum is possible generalizing the one Kähler modulus case discussed in [35]. This result rules out all toroidal orbifold limits with only Kähler moduli for a KKLT scenario, as e.g. the $\mathbf{Z}_{7}$-orbifold. In fact, going beyond the orbifold limit in Ref. [31], we will give the criteria under which a KKLT scenario

may be possible with only Kähler moduli. Furthermore, in subsection 3.6, we find a more general effective superpotential (compared to the ones discussed in [35]) after integrating out several complex structure moduli. Finally, the conditions and solutions for the extrema are presented.

\subsection{Supersymmetry conditions}

In this subsection, we shall study the SUSY conditions for the $\mathbf{Z}_{2} \times \mathbf{Z}_{2}$ orientifold, with $h_{(1,1)}^{\text {untw. }}=h_{(2,1)}^{\text {untw. }}=3$. They read

$$
D_{i} W \equiv \partial_{i} W+\kappa_{4}^{2} W \partial_{i} K=0, \quad i=S, U^{i}, T^{i}
$$

and allow us to explore the Hodge structure of the flux $G_{3}$ in the supersymmetric case. The Kähler potential for the dilaton $S$ and Kähler moduli $T^{i}$ is given in (2.32), while for the complex structure moduli $U^{i}$ it may be read off from (2.7). With the superpotential (3.1) the conditions (3.4) lead to:

$$
\begin{aligned}
D_{T^{i}} W=0 & \Longrightarrow \frac{\lambda}{(2 \pi)^{2} \alpha^{\prime}} \int G_{3} \wedge \Omega=-\left(T^{i}+\bar{T}^{i}\right) g^{i} h^{i} e^{-h^{i} T^{i}}-\sum_{j=1}^{3} g^{j} e^{-h^{j} T^{j}}, \quad i=1,2,3, \\
D_{S} W=0 & \Longrightarrow \frac{\lambda}{(2 \pi)^{2} \alpha^{\prime}} \int \bar{G}_{3} \wedge \Omega=-\sum_{i=1}^{3} g^{i} e^{-h^{i} T^{i}}, \\
D_{U^{i}} W=0 & \Longrightarrow \frac{\lambda}{(2 \pi)^{2} \alpha^{\prime}} \int G_{3} \wedge \omega_{A i}=-\sum_{j=1}^{3} g^{j} e^{-h^{j} T^{j}}, \quad i=1,2,3 .
\end{aligned}
$$


After writing $G_{3}$ in the complex basis ( $c f . E q .(2.67)$ )

$$
\frac{1}{(2 \pi)^{2} \alpha^{\prime}} G_{3}=\sum_{i=0}^{3}\left(A^{i} \omega_{A i}+B^{i} \omega_{B i}\right)
$$

where $\omega_{A 0}$ is a $(3,0)$-form, $\omega_{A i}$ are $(2,1)$-forms, $\omega_{B 0}$ is a $(0,3)$-form and $\omega_{B i}$ are $(1,2)$ forms, we obtain from (3.5):

$$
\begin{aligned}
& B^{0}=-\frac{1}{\lambda \prod_{k=1}^{3}\left(U^{k}+\bar{U}^{k}\right)}\left[\left(T^{i}+\bar{T}^{i}\right) g^{i} h^{i} e^{-h T^{i}}+\sum_{j=1}^{3} g^{j} e^{-h^{j} T^{j}}\right], \quad i=1,2,3, \\
& A^{0}=-\frac{1}{\lambda} \frac{\sum_{i=1}^{3} g^{i} e^{-h^{i} \bar{T}^{i}}}{\prod_{j=1}^{3}\left(U^{j}+\bar{U}^{j}\right)} \quad, \quad B^{i}=-\frac{1}{\lambda} \frac{\sum_{k=1}^{3} g^{k} e^{-h^{k} T^{k}}}{\prod_{j=1}^{3}\left(U^{j}+\bar{U}^{j}\right)}, \quad i=1,2,3 .
\end{aligned}
$$

Here we have used $\int_{X_{6}} \omega_{A_{0}} \wedge \omega_{B_{0}}=\int_{X_{6}} \omega_{B_{i}} \wedge \omega_{A_{i}}=\prod_{k=1}^{3}\left(U^{k}+\bar{U}^{k}\right)$. We see that in the presence of the non-perturbative term the $(1,2),(0,3)$ and $(3,0)$-components of the flux are no longer vanishing. Next, with the formula [54]

$$
\bar{F}^{\bar{I}}=e^{\kappa_{4}^{2} / 2 K} K^{\bar{I} J}\left(\partial_{J} W+\kappa_{4}^{2} W \partial_{J} K\right)
$$

we present the $F$-terms:

$$
\begin{aligned}
\bar{F}^{\bar{S}}= & (S+\bar{S})^{\frac{1}{2}} \prod_{i=1}^{3}\left(T^{i}+\bar{T}^{i}\right)^{-\frac{1}{2}} \prod_{j=1}^{3}\left(U^{j}+\bar{U}^{j}\right)^{-\frac{1}{2}} \kappa_{4}^{2}\left(\frac{\lambda}{(2 \pi)^{2} \alpha^{\prime}} \int \bar{G}_{3} \wedge \Omega+\sum_{k=1}^{3} g^{k} e^{-h^{k} T^{k}}\right), \\
\bar{F}^{\bar{U}^{i}}= & (S+\bar{S})^{-\frac{1}{2}}\left(U^{i}+\bar{U}^{i}\right)^{\frac{1}{2}}\left(U^{j}+\bar{U}^{j}\right)^{-\frac{1}{2}}\left(U^{k}+\bar{U}^{k}\right)^{-\frac{1}{2}} \prod_{l=1}^{3}\left(T^{l}+\bar{T}^{l}\right)^{-\frac{1}{2}} \times \\
& \times \kappa_{4}^{2}\left(\frac{\lambda}{(2 \pi)^{2} \alpha^{\prime}} \int G_{3} \wedge \omega_{A i}+\sum_{m=1}^{3} g^{m} e^{-h^{m} T^{m}}\right), \\
\bar{F}^{T^{i}}= & (S+\bar{S})^{-\frac{1}{2}}\left(T^{i}+\bar{T}^{i}\right)^{\frac{1}{2}}\left(T^{j}+\bar{T}^{j}\right)^{-\frac{1}{2}}\left(T^{k}+\bar{T}^{k}\right)^{-\frac{1}{2}} \prod_{l=1}^{3}\left(U^{l}+\bar{U}^{l}\right)^{-\frac{1}{2}} \times \\
& \times \kappa_{4}^{2}\left[W_{\text {flux }}+\left(T^{i}+\bar{T}^{i}\right) g^{i} h^{i} e^{-h^{i} T^{i}}\right], \quad i \neq j \neq k .
\end{aligned}
$$

With [54

$$
V=K_{I \bar{J}} F^{I} \bar{F}^{\bar{J}}-3 e^{\kappa_{4}^{2} K} \kappa_{4}^{2}|W|^{2}
$$


the potential becomes:

$$
\begin{aligned}
V= & \kappa_{4}^{2}\left(|S+\bar{S}| \prod_{j=1}^{3}\left(T^{j}+\bar{T}^{j}\right) \prod_{k=1}^{3}\left(U^{k}+\bar{U}^{k}\right)\right)^{-1} \times \\
& \times\left\{\sum_{i=1}^{3}\left|W_{\text {flux }}+\left(T^{i}+\bar{T}^{i}\right) g^{i} h^{i} e^{-h^{i} T^{i}}\right|^{2}+\mid \frac{\lambda}{(2 \pi)^{2} \alpha^{\prime}} \int \bar{G}_{3} \wedge \Omega+\sum_{l=1}^{3} g^{l} e^{-\left.h^{l} T^{l}\right|^{2}}\right. \\
& \left.+\sum_{l=1}^{3}\left|\frac{\lambda}{(2 \pi)^{2} \alpha^{\prime}} \int G_{3} \wedge \omega_{A l}+\sum_{m=1}^{3} g^{m} e^{-h^{m} T^{m}}\right|^{2}-3|W|^{2}\right\} .
\end{aligned}
$$

Using (3.6) we can rewrite the potential as:

$$
\begin{aligned}
V= & \kappa_{4}^{2}\left(|S+\bar{S}| \prod_{i=1}^{3}\left|T^{i}+\bar{T}^{i}\right| \prod_{j=1}^{3}\left|U^{j}+\bar{U}^{j}\right|\right)^{-1}\left\{-3 \mid B^{0} \lambda \prod_{l=1}^{3}\left(U^{l}+\bar{U}^{l}\right)+\sum_{l=1}^{3} g^{l} e^{-\left.h^{l} T^{l}\right|^{2}}\right. \\
& +\sum_{k=1}^{3}\left|B^{0} \lambda \prod_{l=1}^{3}\left(U^{l}+\bar{U}^{l}\right)+\sum_{l=1}^{3} g^{l} e^{-h^{l} T^{l}}+\left(T^{k}+\bar{T}^{k}\right) g^{k} h^{k} e^{-h^{k} T^{k}}\right|^{2} \\
& +\left|\lambda \prod_{n=1}^{3}\left(U^{n}+\bar{U}^{n}\right) \bar{A}^{0}+\sum_{p=1}^{3} g^{p} e^{-h^{p} T^{p}}\right|^{2} \\
& \left.+\sum_{r=1}^{3}\left|\lambda \prod_{n=1}^{3}\left(U^{n}+\bar{U}^{n}\right) B^{r}+\sum_{p=1}^{3} g^{p} e^{-h^{p} T^{p}}\right|^{2}\right\} .
\end{aligned}
$$

In the supersymmetric case, i.e. $F^{S}=F^{U^{j}}=F^{T^{i}}=0$, the potential reduces to:

$$
V_{0}=-3 \kappa_{4}^{2} \frac{\left|B^{0} \lambda \prod_{l=1}^{3}\left(U^{l}+\bar{U}^{l}\right)+\sum_{l=1}^{3} g^{l} e^{-h^{l} T^{l}}\right|^{2}}{|S+\bar{S}| \prod_{i=1}^{3}\left|T^{i}+\bar{T}^{i}\right| \prod_{j=1}^{3}\left|U^{j}+\bar{U}^{j}\right|}
$$

Next, we plug the superpotential (3.1) ( $c f$. also [14] for $W_{\text {flux }}$ )

$$
\begin{aligned}
W= & \left(a^{0}+i S c^{0}\right) U^{1} U^{2} U^{3}-\left\{\left(a^{1}+i S c^{1}\right) U^{2} U^{3}+\left(a^{2}+i S c^{2}\right) U^{1} U^{3}+\left(a^{3}+i S c^{3}\right) U^{1} U^{2}\right\} \\
& -\sum_{i=3}^{3}\left(b_{i}+i S d_{i}\right) U^{i}-\left(b_{0}+i S d_{0}\right)+\sum_{i} g^{i} e^{-h^{i} T^{i}}
\end{aligned}
$$


into Eqs. (3.5). The equations become:

$$
\begin{aligned}
& 0=\bar{U}^{1} \bar{U}^{2} \bar{U}^{3}\left(a^{0}+i S c^{0}\right)-\sum_{i \neq j \neq k}\left(a^{i}+i S c^{i}\right) \bar{U}^{j} \bar{U}^{k}-\left(b_{0}+i S d_{0}\right) \\
& -\sum_{i=1}^{3}\left(b_{i}+i S d_{i}\right) \bar{U}^{i}+\sum_{i=1}^{3} g^{i} e^{-h^{i} \bar{T}^{i}} \\
& 0=U^{1} U^{2} U^{3}\left(a^{0}+i S c^{0}\right)-\sum_{i \neq j \neq k}\left(a^{i}+i S c^{i}\right) U^{j} U^{k}-\left(b_{0}+i S d_{0}\right) \\
& -\sum_{i=1}^{3}\left(b_{i}+i S d_{i}\right) U^{i}+\sum_{j=1}^{3} g^{j} e^{-h^{j} T^{j}}+g^{i} h^{i}\left(T^{i}+\bar{T}^{i}\right) e^{-h^{i} T^{i}}, i=1,2,3, \\
& 0=\bar{U}^{1} U^{2} U^{3}\left(a^{0}+i S c_{0}\right)-\left\{\left(a^{1}+i S c^{1}\right) U^{2} U^{3}+\left(a^{2}+i S c^{2}\right) \bar{U}^{1} U^{3}+\left(a^{3}+i S c^{3}\right) \bar{U}^{1} U^{2}\right\} \\
& -\left(b_{0}+i S d_{0}\right)-\left\{\left(b_{1}+i S d_{1}\right) \bar{U}^{1}+\left(b_{2}+i S d_{2}\right) U^{2}+\left(b_{3}+i S d_{3}\right) U^{3}\right\}+\sum_{i=1}^{3} g^{i} e^{-h^{i} T^{i}}, \\
& 0=U^{1} \bar{U}^{2} U^{3}\left(a^{0}+i S c^{0}\right)-\left\{\left(a^{1}+i S c^{1}\right) \bar{U}^{2} U^{3}+\left(a^{2}+i S c^{2}\right) U^{1} U^{3}+\left(a^{3}+i S c^{3}\right) U^{1} \bar{U}^{2}\right\} \\
& -\left(b_{0}+i S d_{o}\right)-\left\{\left(b_{1}+i S d_{1}\right) U^{1}+\left(b_{2}+i S d_{2}\right) \bar{U}^{2}+\left(b_{3}+i S d_{3}\right) U^{3}\right\}+\sum_{i=1}^{3} g^{i} e^{-h^{i} T^{i}}, \\
& 0=U^{1} U^{2} \bar{U}^{3}\left(a^{0}+i S c^{0}\right)-\left\{\left(a^{1}+i S c^{1}\right) U^{2} \bar{U}^{3}+\left(a^{2}+i S c^{2}\right) U^{1} \bar{U}^{3}+\left(a^{3}+i S c^{3}\right) U^{1} U^{2}\right\} \\
& -\left(b_{0}+i S d_{0}\right)-\left\{\left(b_{1}+i S d_{1}\right) U^{1}+\left(b_{2}+i S d_{2}\right) U^{2}+\left(b_{3}+i S d_{3}\right) \bar{U}^{3}\right\}+\sum_{i=1}^{3} g^{i} e^{-h^{i} T^{i}} \text {. }
\end{aligned}
$$

These are the equations to be satisfied at the supersymmetric point of the moduli space.

\subsection{Orientifolds without complex structure modulus}

Let us now discuss the vacuum structure of orientifold compactifications without any complex structure moduli, i.e. $h_{(2,1)}=0$. So the moduli fields which we want to determine by the supersymmetry conditions are the dilaton $S$ and the Kähler moduli $T^{i}$

$\left(i=1, \ldots, h_{(1,1)}^{\text {untw. }}\right)$. Since $\omega_{A_{0}}$ and $\omega_{B_{0}}$ are the only non-trivial 3 -forms, the flux $G_{3}$, expressed in the complex basis, reads:

$$
\frac{1}{(2 \pi)^{2} \alpha^{\prime}} G_{3}=G_{(3,0)}+G_{(0,3)}=A^{0}(S) \omega_{A_{0}}+B^{0}(S) \omega_{B_{0}}
$$

$B^{0}(S)$ is a linear function in $S$ with complex coefficients $B_{1}^{0}, B_{2}^{0}$ :

$$
B^{0}(S)=B_{1}^{0}-i S B_{2}^{0}
$$


The precise form of the $B_{K}^{0}(K=1,2)$ depends on the considered orbifold, as we will discuss in the following. However, the other flux coefficient $A^{0}(S)$ is not anymore an independent function, but it is given as

$$
A^{0}(S)=\bar{B}_{1}^{0}+i S \bar{B}_{2}^{0} .
$$

The flux superpotential which contains the contribution from the $G_{3}$ flux as well as the non-perturbative Kähler moduli dependent term, is given in $E q$. (3.1). Inserting $G_{3}$ of $E q$. (3.16), $W$ becomes:

$$
W=\lambda\left(B_{1}^{0}-i S B_{2}^{0}\right)+\sum_{i=1}^{3} g e^{-h^{i} T^{i}} .
$$

This superpotential is of the same structure as the superpotential discussed in [35] (see section 3.1 in that paper). The main difference to the superpotential of 35] is that here, the coefficients $B_{1}^{0}$ and $B_{2}^{0}$ have a microscopic explanation in terms of 3-form flux quantum numbers. It follows that these coefficients are integer-valued. Hence the flux quantization will put some additional constraints on the allowed solutions of the supersymmetry equations.

Let us consider in more detail the $\mathbf{Z}_{3} \times \mathbf{Z}_{3}$ orbifold. Here the complex flux coefficients read (see appendix B.7.):

$$
B_{1}^{0}=\frac{1}{\sqrt{3}}\left(i a^{1}+e^{-5 \pi i / 6} b_{1}\right), \quad B_{2}^{0}=\frac{1}{\sqrt{3}}\left(i c^{1}+e^{-5 \pi i / 6} d_{1}\right), \quad a^{1}, b_{1}, c^{1}, d_{1} \in \mathbf{Z} .
$$

In order to determine the exact form of the flux part of the superpotential, we also need the prefactor $\lambda$. For the $\mathbf{Z}_{3} \times \mathbf{Z}_{3}$ orbifold it takes the value $\lambda=i \sqrt{3}$ [53].

Now we may determine the solutions of the two supersymmetry conditions $D_{T} W=0$ and $D_{S} W=0$. We may essentially follow the procedure outlined in 35. We shall consider the simplified case where all Kähler moduli $T^{i}$ are identified, i.e. $T^{i}=T$, and also $h^{i}=h$. Now observe that via a field redefinition in $T$, namely a constant shift in $\operatorname{Im} T$, the coefficient $g$ can always be chosen to be real. Similarly one can shift $\operatorname{Im} S$, such that $i \sqrt{3} B_{1}^{0}$ is real. So we choose $b_{1}=0$ in eq.(3.20). For simplicity we also choose $i \sqrt{3} B_{2}^{0}$ to be real. Taking all this into account, the superpotential (3.19) becomes:

$$
W=-a^{1}+\frac{\sqrt{3}}{2} d_{1} S+3 g e^{-h T}, \quad a^{1}, d_{1} \in \mathbf{Z} .
$$

As in [35], we may restrict the analysis to the case where the moduli $S$ and $T$ are purely real, i.e. $T=t$ and $S=s$. Then the two supersymmetry conditions provide the following two constraints on $s$ and $t$ :

$$
a^{1}=g e^{-h t}(h t+3), \quad \frac{d_{1}}{a^{1}}=-\frac{2 h t}{\sqrt{3} s(h t+3)} .
$$


Since $e^{-h t}(h t+3) \leq 3$, it follows that the first equation has only solutions for integer values of $a^{1}$, if the parameter $|g| \geq 1 / 3$. In fact due to charge quantization, for any given $|g| \geq 1 / 3$, this equation has a finite number of allowed solutions (for $|g|=1 / 3$ the solution occurs at $t=0$ ). Specifically, the first equation possesses solutions in $t$ for the following values of the flux $a^{1}$ :

$$
a^{1}=1, \ldots,\left[g^{\prime}\right], \quad g^{\prime}=3 g .
$$

Here we have assumed that $g>0$, otherwise $a^{1}<0$. Finally, after having solved the first constraint in (3.22) which fixes the modulus $t$, the second equation does not put any further conditions on the allowed fluxes, it possesses precisely one solution in $s$ for any given choice of $a^{1}, c^{1}$. Let us assume that $g$ is very large, $|g|>>\left|a^{1}\right|$. Then the supersymmetry condition is solved for very large $t$. Furthermore, if we insist on weak string coupling, i.e. large $s$, we have to demand that $\left|a^{1}\right|>>\left|c^{1}\right|$.

As discussed in [35], the above solutions of the two supersymmetry conditions do not correspond to stable supersymmetric vacua, but the supersymmetric point rather is a saddle point with instabilities along the moduli and axionic directions. Hence, we like to proceed to consider orbifolds with more than one Kähler modulus and/or complex structure moduli in order to see whether stable supersymmetric ground states now become possible.

\subsection{Orientifolds with three Kähler moduli and fixed complex structure moduli}

After having discussed the case of one Kähler modulus in the previous subsection, we now shall move on to the three Kähler moduli case. This case captures e.g. the $\mathbf{Z}_{7}$-orbifold. We start with the following ansatz for the superpotential (3.1)

$$
W=\alpha_{1}+\alpha_{2} S+\sum_{j=1}^{3} g^{j} e^{-h^{j} T^{j}},
$$

with complex coefficients $\alpha_{1}=B_{1}^{0}, \alpha_{2}=-i B_{2}^{0}, g^{j}$ and $h^{j}>0$. With the Kähler potential

$$
\kappa_{4}^{2} K=-\ln (S+\bar{S})-\sum_{j=1}^{3} \ln \left(T^{j}+\bar{T}^{j}\right)
$$

for the closed string moduli sector we derive the following $F$-terms:

$$
\begin{aligned}
-(S+\bar{S})^{-1 / 2} \prod_{i=1}^{3}\left(T^{i}+\bar{T}^{i}\right)^{1 / 2} \bar{F}^{S} & =\alpha_{1}-\alpha_{2} \bar{S}+\sum_{j=1}^{3} g^{j} e^{-h^{j} T^{j}} \\
-\frac{(S+\bar{S})^{1 / 2}\left(T^{i}+\bar{T}^{i}\right)^{1 / 2}\left(T^{k}+\bar{T}^{k}\right)^{1 / 2}}{\left(T^{j}+\bar{T}^{j}\right)^{1 / 2}} \bar{F}^{T^{j}} & =h^{j} g^{j}\left(T^{j}+\bar{T}^{j}\right) e^{-h^{j} T^{j}}+\alpha_{1}+\alpha_{2} S \\
& +\sum_{j=1}^{3} g^{j} e^{-h^{j} T^{j}} \quad, \quad(i, j, k)=\overline{(1,2,3)}
\end{aligned}
$$


and similarly for their complex conjugate $F^{T^{j}}$ and $F^{S}$. Demanding $F^{S}=0=F^{T^{j}}$ leads to the following relations:

$$
\alpha_{1}=\alpha_{2}\left(\bar{S}+\sum_{j=1}^{3} \frac{S+\bar{S}}{h^{j}\left(T^{j}+\bar{T}^{j}\right)}\right) \quad, \quad g^{j}=-\frac{\alpha_{2} e^{h^{j} T^{j}}}{h^{j}} \frac{S+\bar{S}}{T^{j}+\bar{T}^{j}}, j=1,2,3,
$$

and their complex conjugate. These relations have to be obeyed at the extremum of the potential. In principle, the point $\left(S_{0}, T_{0}^{j}\right)$ of the extremum may be determined from these relations (3.27). It is straightforward to calculate the scalar potential $V\left(S, T^{j}\right)$. At the extremum $\left(S_{0}, T_{0}^{j}\right)$, its value is given by

$$
V_{0}=-3 \frac{\left|\alpha_{2}\right|^{2}\left(S_{0}+\bar{S}_{0}\right)}{\prod_{j=1}^{3}\left(T_{0}^{j}+\bar{T}_{0}^{j}\right)}
$$

To determine the kind of extremum, we have to calculate the second derivatives of the potential $V\left(S, T^{j}\right)$ w.r.t. the moduli fields. It is convenient to introduce $S=s_{1}+i s_{2}$ and $T^{j}=t_{1}^{j}+i t_{2}^{j}$. W.r.t. the parameters $s_{i}, t_{i}^{j}$ we find the following identities for the mixed derivatives:

$$
\frac{\partial^{2} V}{\partial s_{1} \partial t_{2}^{j}}=\frac{\partial^{2} V}{\partial t_{1}^{j} \partial s_{2}}=\frac{\partial^{2} V}{\partial s_{1} \partial s_{2}}=\frac{\partial^{2} V}{\partial t_{1}^{k} \partial t_{2}^{l}}=0
$$

On the other hand, the non-vanishing components of the Hessian $H=\left(\begin{array}{cc}H_{1} & 0 \\ 0 & H_{2}\end{array}\right)$ are arranged in a block-form with two $4 \times 4$ matrices $H_{1}$ and $H_{2}$, with their determinants given by:

$$
\begin{aligned}
\operatorname{det} H_{1} & =-\frac{s_{1}^{2}\left|\alpha_{2}\right|^{8}}{512\left(t_{1}^{1} t_{1}^{2} t_{1}^{3}\right)^{6}}\left(2+h^{1} h^{2} h^{3} t_{1}^{1} t_{1}^{2} t_{1}^{3}-\sum_{j=1}^{3} h^{j} t_{1}^{j}\right) \\
& \times\left(5+16 h^{1} h^{2} h^{3} t_{1}^{1} t_{1}^{2} t_{1}^{3}+8 \sum_{j=1}^{3} h^{j} t_{1}^{j}+6 \sum_{i \neq j} h^{i} h^{j} t_{1}^{i} t_{1}^{j}\right), \\
\operatorname{det} H_{2} & =-\frac{h^{1} h^{2} h^{3} s_{1}^{2}\left|\alpha_{2}\right|^{8}}{512\left(t_{1}^{1} t_{1}^{2} t_{1}^{3}\right)^{5}}\left(27+16 h^{1} h^{2} h^{3} t_{1}^{1} t_{1}^{2} t_{1}^{3}-6 \sum_{i \neq j} h^{i} h^{j} t_{1}^{i} t_{1}^{j}\right) .
\end{aligned}
$$

The latter may become positive in a certain region of the parameter space $h^{i} t_{1}^{i}$. In order for $H_{1}$ and $H_{2}$ to be positive definite, also their subdeterminants have to be positive, i.e.

$$
\begin{gathered}
H_{11}>0, H_{11} H_{22}-H_{12}^{2}>0 \text { and } \operatorname{det}\left(\begin{array}{lll}
H_{11} & H_{12} & H_{13} \\
H_{12} & H_{22} & H_{23} \\
H_{13} & H_{23} & H_{33}
\end{array}\right)>0 . \text { However, we find } \\
\left(H_{1}\right)_{11}\left(H_{1}\right)_{22}-\left(H_{1}\right)_{12}^{2}=-\frac{1}{32\left(t_{1}^{1}\right)^{4}\left(t_{1}^{2}\right)^{2}\left(t_{1}^{3}\right)^{2}}\left|\alpha_{2}\right|^{4}\left(4+5 h^{1} t_{1}^{1}\right)<0,
\end{gathered}
$$


and

$$
\left(H_{2}\right)_{11}\left(H_{2}\right)_{22}-\left(H_{2}\right)_{12}^{2}=-\frac{3}{32\left(t_{1}^{1}\right)^{3}\left(t_{1}^{2}\right)^{2}\left(t_{1}^{3}\right)^{2}}\left|\alpha_{2}\right|^{4} h^{1}<0
$$

and conclude that the extremum $\left(S_{0}, T_{0}^{j}\right)$ is no minimum.

Hence a KKLT scenario is not possible in the $\mathbf{Z}_{7}$-orbifold with only untwisted Kähler moduli. This generalizes the results of [35] for one Kähler modulus to the three Kähler moduli case.

\subsection{Orientifolds with five Kähler moduli and fixed complex structure moduli}

After having found this negative result for three Kähler moduli arising from orbifolds with a diagonal Hermitian complex metric, we shall now investigate the case with nondiagonal metric. We want to discuss the Kähler moduli space $\frac{S U(2,2)}{S U(2) \times S U(2) \times U(1)} \times \frac{S U(1,1)}{U(1)}$ with five Kähler moduli. This moduli space has been parameterized in subsection 2.4 and is relevant to the $\mathbf{Z}_{6-I^{-}}$-orientifolds. In $E q$. (2.45) the Kähler potential has been given.

In order to keep formulae short we shall stick to the case $T^{1}=T^{2}$ and $T^{3}=T^{4}$, though we have performed the analysis for all five Kähler moduli kept arbitrary. So we start with the following ansatz for the superpotential (3.1)

$$
W=\alpha_{1}+\alpha_{2} S+g^{1} e^{-h^{1} T^{1}}+g^{3} e^{-h^{3} T^{3}}+g^{5} e^{-h^{5} T^{5}},
$$

with complex coefficients $\alpha_{1}, \alpha_{2}, g^{j}$ and $h^{j}>0$. With the Kähler potential (2.45)

$$
\kappa_{4}^{2} K=-\ln (S+\bar{S})-\ln \left(T^{5}+\bar{T}^{5}\right)-\ln \left[\left(T^{1}+\bar{T}^{1}\right)^{2}-\left(T^{3}+\bar{T}^{3}\right)^{2}\right]
$$

we calculate the $F$-terms:

$$
\begin{aligned}
\frac{Y^{1 / 2}}{(S+\bar{S})^{1 / 2}} \bar{F}^{\bar{S}} & =-\alpha_{1}+\alpha_{2} \bar{S}-\sum_{j=1,3,5} g^{j} e^{-h^{j} T^{j}}, \\
(S+\bar{S})^{1 / 2} Y^{1 / 2} \bar{F}^{1} & =-\alpha_{1}-\alpha_{2} S-\sum_{j=1,3,5} g^{j} e^{-h^{j} T^{j}} \\
& -\frac{1}{2} g^{1} h^{1} e^{-h^{1} T^{1}}\left[\left(T^{1}+\bar{T}^{1}\right)^{2}+\left(T^{3}+\bar{T}^{3}\right)^{2}\right] \\
& -g^{3} h^{3} e^{-h^{3} T^{3}}\left(T^{1}+\bar{T}^{1}\right)\left(T^{2}+\bar{T}^{2}\right), \\
(S+\bar{S})^{1 / 2} Y^{1 / 2} \bar{F}^{3} & =-\alpha_{1}-\alpha_{2} S-\sum_{j=1,3,5} g^{j} e^{-h^{j} T^{j}} \\
& -g^{1} h^{1} e^{-h^{1} T^{1}}\left(T^{1}+\bar{T}^{1}\right)\left(T^{2}+\bar{T}^{2}\right) \\
& -\frac{1}{2} g^{3} h^{3} e^{-h^{3} T^{3}}\left[\left(T^{1}+\bar{T}^{1}\right)^{2}+\left(T^{3}+\bar{T}^{3}\right)^{2}\right], \\
\frac{(S+\bar{S})^{1 / 2} Y^{1 / 2}}{\left(T^{5}+\bar{T}^{5}\right)} \bar{F}^{5} & =-\alpha_{1}-\alpha_{2} S-g^{5} h^{5} e^{-h^{5} T^{5}}\left(T^{5}+\bar{T}^{5}\right)-\sum_{j=1,3,5} g^{j} e^{-h^{j} T^{j}},
\end{aligned}
$$


with $Y=\left(T^{5}+\bar{T}^{5}\right)\left[\left(T^{1}+\bar{T}^{1}\right)^{2}-\left(T^{3}+\bar{T}^{3}\right)^{2}\right]$. Demanding $F^{S}=0=F^{T^{j}}$ leads to the following relations

$$
\begin{aligned}
& \alpha_{1}=\alpha_{2}\left(\bar{S}+\frac{S+\bar{S}}{h^{5}\left(T^{5}+\bar{T}^{5}\right)}+\frac{2(S+\bar{S})\left(T^{1}+\bar{T}^{1}\right)}{h^{1}\left[\left(T^{1}+\bar{T}^{1}\right)^{2}-\left(T^{3}+\bar{T}^{3}\right)^{2}\right]}-\frac{2(S+\bar{S})\left(T^{3}+\bar{T}^{3}\right)}{h^{3}\left[\left(T^{1}+\bar{T}^{1}\right)^{2}-\left(T^{3}+\bar{T}^{3}\right)^{2}\right]}\right) \\
& g^{1}=-2 \frac{\alpha_{2} e^{h^{1} T^{1}}}{h^{1}} \frac{(S+\bar{S})\left(T^{1}+\bar{T}^{1}\right)}{\left(T^{1}+\bar{T}^{1}\right)^{2}-\left(T^{3}+\bar{T}^{3}\right)^{2}}, g^{3}=2 \frac{\alpha_{2} e^{h^{3} T^{3}}}{h^{3}} \frac{(S+\bar{S})\left(T^{3}+\bar{T}^{3}\right)}{\left(T^{1}+\bar{T}^{1}\right)^{2}-\left(T^{3}+\bar{T}^{3}\right)^{2}}, \\
& g^{5}=-\frac{\alpha_{2} e^{h^{5}} T^{5}}{h^{5}} \frac{S+\bar{S}}{T^{5}+\bar{T}^{5}}
\end{aligned}
$$

to be satisfied at the extremum. At the extremum $\left(S_{0}, T_{0}^{j}\right)$ the scalar potential takes the value:

$$
V_{0}=-3\left|\alpha_{2}\right|^{2} \frac{\left(S_{0}+\bar{S}_{0}\right)}{\left(T_{0}^{5}+\bar{T}_{0}^{5}\right)\left[\left(T_{0}^{1}+\bar{T}_{0}^{1}\right)^{2}-\left(T_{0}^{3}+\bar{T}_{0}^{3}\right)^{2}\right]}
$$

The scalar masses $m_{S}, m_{T^{j}}$ are encoded in the Hessian of the scalar potential $V\left(S, T^{1}, T^{3}, T^{5}\right)$. After introducing $S=s_{1}+i s_{2}$ and $T^{j}=t_{1}^{j}+i t_{2}^{j}$ we calculate the Hessian $H$ of the potential w.r.t. to the eight real variables $s_{1}, s_{2}, t_{1}^{j}, t_{2}^{j}, j=1,3,5$. This gives an $8 \times 8$ matrix, which has to be positive definite in order to guarantee for positive scalar masses. This means, that all upper left matrices of $H$ must have positive determinants. Similarly as before, we determine:

$$
\frac{\partial^{2} V}{\partial s_{1} \partial t_{2}^{j}}=\frac{\partial^{2} V}{\partial t_{1}^{j} \partial s_{2}}=\frac{\partial^{2} V}{\partial s_{1} \partial s_{2}}=\frac{\partial^{2} V}{\partial t_{1}^{k} \partial t_{2}^{l}}=0 .
$$

Hence, the Hessian decomposes into two $4 \times 4$ blocks, which individually have to be positive definite. However, at the extremum $\left(S_{0}, T_{0}^{j}\right)$ we find the following:

$$
\begin{aligned}
& \frac{\partial^{2} V}{\partial s_{2}^{2}}=\frac{\left|\alpha_{2}\right|^{2}}{8} \frac{1}{s_{1} t_{1}^{5}\left[\left(t_{1}^{1}\right)^{2}-\left(t_{1}^{3}\right)^{2}\right]}>0, \\
& \operatorname{det}\left(\begin{array}{cc}
\frac{\partial^{2} V}{\partial\left(s_{2}\right)^{2}} & \frac{\partial^{2} V}{\partial s_{2} \partial t_{2}^{1}} \\
\frac{\partial^{2} V}{\partial t_{2}^{1} \partial s_{2}} & \frac{\partial^{2} V}{\partial\left(t_{2}^{1}\right)^{2}}
\end{array}\right)=-\frac{\left|\alpha_{2}\right|^{4} h^{1} t_{1}^{1}}{16} \frac{\left(3+2 h^{1} t_{1}^{1}\right)}{\left(t_{1}^{5}\right)^{2}\left[\left(t_{1}^{1}\right)^{2}-\left(t_{1}^{3}\right)^{2}\right]^{3}}<0, \\
& \operatorname{det}\left(\begin{array}{ccc}
\frac{\partial^{2} V}{\partial\left(s_{2}\right)^{2}} & \frac{\partial^{2} V}{\partial s_{2} \partial t_{2}^{1}} & \frac{\partial^{2} V}{\partial s_{2} \partial t_{2}^{3}} \\
\frac{\partial^{2} V}{\partial t_{2}^{1} \partial s_{2}} & \frac{\partial^{2} V}{\left.\partial t_{2}^{1}\right)^{2}} & \frac{\partial^{2} V}{\partial t_{2}^{1} \partial t_{2}^{3}} \\
\frac{\partial^{2} V}{\partial t_{2}^{3} \partial s_{2}} & \frac{\partial^{2} V}{\partial t_{2}^{3} \partial t_{2}^{1}} & \frac{\partial^{2} V}{\partial\left(t_{2}^{3}\right)^{2}}
\end{array}\right)=-\frac{\left|\alpha_{2}\right|^{6} h^{1} h^{3} s_{1} t_{1}^{1} t_{1}^{3}}{32} \frac{\left(3+2 h^{1} t_{1}^{1}\right)\left(3+2 h^{3} t_{1}^{3}\right)}{\left(t_{1}^{5}\right)^{3}\left[\left(t_{1}^{1}\right)^{2}-\left(t_{1}^{3}\right)^{2}\right]^{5}}<0 .
\end{aligned}
$$

Since $\left(t_{1}^{1}\right)^{2}-\left(t_{1}^{3}\right)^{2}>0$ (cf. subsection 2.4) and $h^{j}>0$, we conclude, that there is no parameter range $g^{j}, h^{j}$, for which our exremum becomes a minimum. This continues to 
hold for all five Kähler moduli $T^{j}$ kept arbitrary. In that case in the Hessian we encounter e.g. the submatix

$$
\operatorname{det}\left(\begin{array}{cc}
\frac{\partial^{2} V}{\partial\left(s_{2}\right)^{2}} & \frac{\partial^{2} V}{\partial s_{2} \partial t_{2}^{1}} \\
\frac{\partial^{2} V}{\partial t_{2}^{1} \partial s_{2}} & \frac{\partial^{2} V}{\partial\left(t_{2}^{1}\right)^{2}}
\end{array}\right)=-\frac{3\left|\alpha_{2}\right|^{4} h^{1} t_{1}^{2}}{32} \frac{h^{1} t_{1}^{1}}{\left(t_{1}^{5}\right)^{2}\left(t_{1}^{1} t_{1}^{2}-t_{1}^{3} t_{1}^{4}\right)^{3}}<0
$$

which is negative-definite for $t_{1}^{1} t_{1}^{2}-t_{1}^{3} t_{1}^{4}>0$ (cf. subsection 2.4).

Furthermore, due to the similarity of the coset structure of the nine Kähler moduli case $\frac{S U(3,3)}{S U(3) \times S U(3) \times U(1)}$ to the above case, it is expected that no stable minimum can be found either.

\subsection{Orientifolds with one untwisted complex structure modulus}

Now consider orientifolds with one untwisted complex structure modulus, labelled by $U^{3}$. The main issue will be to solve the supersymmetry conditions, taking into account the flux quantization, and to see if in contrast to the previous case there are stable vacua. The relevant 3 -forms are the $(3,0)$-form $\omega_{A_{0}}$ and one $(2,1)$-form $\omega_{A_{3}}$ plus their conjugate $(0,3)$ and $(1,2)$-forms $\omega_{B_{0}}$ and $\omega_{B_{3}}$. In terms of these complex 3 -forms, the flux $G_{3}$ may be expanded as:

$$
\begin{aligned}
\frac{1}{(2 \pi)^{2} \alpha^{\prime}} G_{3} & =G_{(3,0)}+G_{(2,1)}+G_{(0,3)}+G_{(1,2)}= \\
& =A^{0}\left(S, U^{3}\right) \omega_{A_{0}}+A^{3}\left(S, U^{3}\right) \omega_{A_{3}}+B^{0}\left(S, U^{3}\right) \omega_{B_{0}}+B^{3}\left(S, U^{3}\right) \omega_{B_{3}} .
\end{aligned}
$$

Now, the complex coefficients take the form

$$
B^{0}(S)=B_{1}^{0}\left(U^{3}\right)-i B_{2}^{0}\left(U^{3}\right) S, \quad B^{3}(S)=B_{1}^{3}\left(U^{3}\right)-i B_{2}^{3}\left(U^{3}\right) S
$$

where the $B^{0}\left(U^{3}\right), B^{3}\left(U^{3}\right)$ each contain a constant term and a term linear in $U^{3}$. All together they comprise eight real integer valued flux parameters, whose explicit forms depend on the individual orientifold under investigation (see later). Using this 3-form flux, the superpotential (3.1) may be written as

$$
W=\lambda\left[B_{1}^{0}\left(U^{3}\right)-i B_{2}^{0}\left(U^{3}\right) S\right]+\sum_{i=1}^{3} g^{i} e^{-h^{i} T^{i}}
$$

which for convenience we parameterize as:

$$
W=\alpha_{0}+\alpha_{1} U^{3}+\alpha_{2} S+\alpha_{3} S U^{3}+\sum_{i=1}^{3} g^{i} e^{-h^{i} T^{i}}, \quad \alpha_{i} \in \mathbf{R} .
$$


In the following, we consider first the situation, where in the first step the complex structure modulus $U^{3}$ is integrated out; this leads to an effective superpotential $W_{\text {eff }}(S, T)$. In the second step, the supersymmetry conditions $D_{T} W_{\text {eff }}(S, T)=D_{S} W_{\text {eff }}(S, T)=0$ are imposed for the effective superpotential $W_{\text {eff }}(S, T)$. As pointed out in Ref. [35], this procedure is valid as long as the vacuum has the property that the complex structure moduli $U^{i}$ are much heavier than the fields $S$ and $T_{i}$. Assuming that this assumption indeed holds, we consider the supersymmetry condition for $U^{3}$,

$$
D_{U^{3}} W=\alpha_{1}+\alpha_{3} S-\frac{\alpha_{0}+\alpha_{1} U^{3}+\alpha_{2} S+\alpha_{3} S U^{3}+\sum_{i=1}^{3} g^{i} e^{-h^{i} T^{i}}}{U^{3}+\bar{U}^{3}}=0,
$$

and plug back its solution for $U^{3}$ into the superpotential. This results in the following effective superpotential that now depends only on $S$ and $T_{i}$ (for real $U^{3}$ ):

$$
W_{\text {eff }}(S, T)=2\left(\alpha_{0}+\alpha_{2} S+\sum_{i=1}^{3} g^{i} e^{-h^{i} T^{i}}\right) .
$$

We see that this effective superpotential is again a linear function in $S$. In fact, it is precisely of the same structure as the superpotential (3.19) of the previous section without complex structure modulus. Hence all conclusions about the vacuum structure with respect to $S$ and $T$ are unchanged. In particular, the supersymmetric stationary points in $S$ and $T$ are not stable ground states with a positive definite moduli mass matrix. This result has already been obtained in 35.

Alternatively, we can also determine the solutions of all supersymmetry conditions $D_{U^{3}} W=D_{S} W=D_{T} W=0$ at the same time without first integrating out $U^{3}$. For simplicity we consider the isotropic case $T:=T^{1}=T^{2}=T^{3}, h_{1}=h_{2}=h_{3}$ and real flux parameters $\alpha_{i}$. We write the moduli fields as $T=t+i \tau, S=s+i \sigma$ and $U^{3}=u_{3}+i \nu$. To make the calculation clear, we confine ourselves to the supersymmetric point with $\sigma=0$, $\nu=0, \tau=0$. The constraints which have to be fulfilled at the supersymmetric point become:

$$
\begin{aligned}
s & =-\frac{1}{\alpha_{2}}\left(\alpha_{0}+(3+h t) g e^{-h t}\right), \\
u_{3} & =-\frac{\alpha_{2}}{\alpha_{3}}\left(\frac{\alpha_{0}+3 g e^{-h t}}{\alpha_{0}+(3+h t) g e^{-h t}}\right), \\
\alpha_{1} & =\frac{\alpha_{3}\left(\alpha_{0}+g e^{-h t}(3+h t)\right)^{2}}{\alpha_{2}\left(\alpha_{0}+3 g e^{-h t}\right)} .
\end{aligned}
$$

Here, $s$ and $u_{3}$ are the real parts of the dilaton and complex structure moduli respectively, and should be positive. From the above constraints we see that this excludes some values 
for $\alpha_{0}, \alpha_{2}$ and $\alpha_{3}$. If we allow for $t$ every positive value, the situation is simple. One has two possibilities

$$
\alpha_{0} \geq 0, \quad \alpha_{2}<0, \quad \alpha_{3}>0
$$

and

$$
\alpha_{0}<-(3+h t) g e^{-h t}, \quad \alpha_{2}>0, \quad \alpha_{3}<0 .
$$

In the same way as in the previous section we compute the potential and then calculate the second derivatives at the supersymmetric point. This means that we plug the constraints (3.45) into the matrix of second derivatives. The resulting six-dimensional matrix is of block diagonal form (two blocks $3 \times 3$ ). The condition for the supersymmetric point to be a minimum is that the diagonal blocks should be positive definite. This requirement may be translated into the statement that the determinants associated with all upper-left submatrices are positive. We abbreviate the sub-determinants of the upper block by $a_{11}$, $a_{22}, a_{33}$ and those of the lower block by $a_{44}, a_{55}, a_{66}$. They are

$$
\begin{aligned}
a_{11}= & \frac{\alpha_{3}^{3}}{8 \alpha_{2}^{2} t^{3}\left(\alpha_{0}+3 g e^{-h t}\right)^{3}}\left(\alpha_{0}+(3+h t) g e^{-h t}\right)^{2} \times \\
& \times\left(2 \alpha_{0}^{2}+2 \alpha_{0}(6+h t) g e^{-h t}+g^{2} e^{-2 h t}\left(18+6 h t+h^{2} t^{2}\right)\right), \\
a_{22}= & \frac{\alpha_{3}^{4}}{64 t^{6}\left(\alpha_{0}+3 g e^{-h t}\right)^{4}}\left(2 \alpha_{0}^{2}+\alpha_{0}(12+h t) g e^{-h t}+g^{2} e^{-2 h t}\left(18+3 h t-h^{2} t^{2}\right)\right) \times \\
& \times\left(2 \alpha_{0}^{2}+3 \alpha_{0}(4+h t) g e^{-h t}+3 g^{2} e^{-2 h t}\left(6+3 h t+h^{2} t^{2}\right)\right), \\
a_{33}= & \frac{3 \alpha_{3}^{5} h^{2} g^{2} e^{-2 h t}}{512 t^{9}\left(\alpha_{0}+3 g e^{-h t}\right)^{5}}\left(2 \alpha_{0}^{2}+\alpha_{0}(12+h t) g e^{-h t}+g^{2} e^{-2 h t}\left(18+3 h t-h^{2} t^{2}\right)\right) \times \\
& \times\left(\alpha_{0}(1+2 h t)+3 g e^{-h t}(1+h t)\right)\left(2 \alpha_{0}(2+h t)+3 g e^{-h t}\left(4+3 h t+h^{2} t^{2}\right)\right), \\
a_{44}= & \frac{\alpha_{3}^{3}}{8 \alpha_{2}^{2} t^{3}\left(\alpha_{0}+3 g e^{-h t}\right)^{3}}\left(\alpha_{0}+(3+h t) g e^{-h t}\right)^{2} \times \\
& \times\left(2 \alpha_{0}^{2}+2 \alpha_{0}(6+h t) g e^{-h t}+g^{2} e^{-2 h t}\left(18+6 h t+h^{2} t^{2}\right)\right), \\
a_{55}= & \frac{\alpha_{3}^{4}}{64 t^{6}\left(\alpha_{0}+3 g e^{-h t}\right)^{3}}\left(2 \alpha_{0}+3(2 h+t) g e^{-h t}\right) \times \\
& \times\left(2 \alpha_{0}^{2}+\alpha_{0}(12+h t) g e^{-h t}+g^{2} e^{-2 h t}\left(18+3 h t+2 h^{2} t^{2}\right)\right), \\
a_{66}= & \frac{3 \alpha_{3}^{5} h^{3} g^{2} e^{-2 h t}}{512 t^{8}\left(\alpha_{0}+3 g e^{-h t}\right)^{4}}\left(4 \alpha_{0}^{2}+2 \alpha_{0}(9+2 h t) g e^{-h t}+\left(18+3 h t-3 h^{2} t^{2}\right) g^{2} e^{-2 h t}\right) \times \\
& \times\left(\alpha_{0}(3+2 h t)+\left(9+6 h t+2 h^{2} t^{2}\right) g e^{-h t}\right) .
\end{aligned}
$$

To analyze these minors we have to distinguish the two cases (3.46) und (3.47). 
In the first case (3.46), the conditions for the positivity of the minors are

$$
\begin{aligned}
& 2 \alpha_{0}^{2}+\alpha_{0}(12+h t) g e^{-h t}+\left(18+3 h t-h^{2} t^{2}\right) g^{2} e^{-2 h t}>0 \\
& 4 \alpha_{0}^{2}+2 \alpha_{0}(9+2 h t) g e^{-h t}+\left(18+3 h t-3 h^{2} t^{2}\right) g^{2} e^{-2 h t}>0 .
\end{aligned}
$$

In the case for vanishing $\alpha_{0}$ we obtain $h t<3$. In other cases the term $\alpha_{0}^{2}$ is dominant for large $t$ and (3.49) is true. For the small $t$, the values of $a_{22}, a_{33}$ and $a_{66}$ could be negative. However, this depends on the values of $g$ and $h$.

In the second case (3.47), the conditions are the same (3.49), with the difference that $\alpha_{0}<-(3+h t) g e^{-h t}$. It means all minors are positive for large $t$ as in the previous case.

To conclude, stable minima do exist for orbifolds with one complex structure modulus. In addition, we see that there is a discrepancy between whether we integrate out the complex structure modulus or not. The reason for this discrepancy is that the complex structure modulus is not heavy and therefore is not allowed to be simply integrated out.

Finally, we give an example which falls into class (3.46) of the solutions. This example is $Z_{6-I I}$ on $(S U(2))^{2} \times S U(3) \times G_{2}$. The superpotential is given by:

$$
\begin{aligned}
W= & -i e^{-\pi i / 6} b_{0}+b_{2}+S\left(i e^{-\pi i / 6} d_{0}-d_{2}\right) \\
& -U^{3}\left[\frac{i}{\sqrt{3}} e^{-\pi i / 3} a_{0}+\frac{i}{\sqrt{3}} a_{2}-S\left(\sqrt{3} e^{-2 \pi i / 6} c_{0}+\frac{1}{\sqrt{3}} c_{2}\right)\right]+g e^{-h T} .
\end{aligned}
$$

We choose $b_{0}=d_{0}=c_{0}=0$ and $a_{2}=-\frac{1}{2} a_{0}$. Further $a_{0}, b_{2}, c_{2}$ and $d_{2}$ should be positive. In this case, we obtain a superpotential of the form (3.42).

\subsection{Orientifolds with three untwisted complex structure moduli}

In the $\mathbf{Z}_{2} \times \mathbf{Z}_{2}$ orientifold, we have three untwisted complex structure moduli $U^{i}$ undetermined. In this case, all $\omega_{A_{i}}$ and $\omega_{B_{i}}$ survive, and the (primitive) 3-form flux takes the following form:

$$
\frac{1}{(2 \pi)^{2} \alpha^{\prime}} G_{3}=\sum_{i=0}^{3}\left[A^{i}\left(S, U^{i}\right) \omega_{A_{i}}+B^{i}\left(S, U^{i}\right) \omega_{B_{i}}\right] .
$$

The corresponding superpotential (3.1) becomes:

$$
W=\lambda\left[B_{1}^{0}\left(U^{i}\right)-i B_{2}^{0}\left(U^{i}\right) S\right]+\sum_{i=1}^{3} g^{i} e^{-h^{i} T^{i}} .
$$

The coefficients $B_{1,2}^{0}$ are determined by 16 integer valued flux quantum numbers. The supersymmetry conditions for this superpotential with seven moduli fields and 16 flux quantum numbers have been given in (3.15). However, it is very involved to solve them in 
a closed form. Therefore, we reduce the number of fields and parameters by setting two of the complex structure moduli equal to each other, e.g. $U^{1}=U^{2}$. Then the superpotential is somewhat simpler and may be parameterized by eight integer valued fluxes $\alpha_{j}(j=0, \ldots, 7)$ in the following way:

$$
W=\alpha_{0}+\alpha_{1} U^{1}+\alpha_{2} U^{3}+\alpha_{3} S+\alpha_{4} S U^{1}+\alpha_{5} S U^{3}+\alpha_{6} U^{1} U^{3}+\alpha_{7} S U^{1} U^{3}+\sum_{i=1}^{3} g^{i} e^{-h^{i} T^{i}}
$$

In this case, the effective Kähler potential is given by (cf. section 2):

$$
\kappa_{4}^{2} K=-\ln (S+\bar{S})-2 \ln \left(U^{1}+\bar{U}^{1}\right)-\ln \left(U^{3}+\bar{U}^{3}\right)-\sum_{i=1}^{3} \ln \left(T^{i}+\bar{T}^{i}\right)
$$

In order to determine the vacuum structure of this class of models we will first use the integrating out procedure for all three complex structure moduli, assuming that they are heavy compared to $S$ and $T^{i}$. Again, the aim of this investigation is to see, whether there are stable supersymmetric vacua with positive definite mass matrix in $S$ and $T$ or not. Hence, we consider the two supersymmetry conditions $D_{U^{1,2}} W=0$. Their solution becomes (for real $U^{i}$ ):

$$
\begin{aligned}
& U^{1}=\frac{-2\left(\alpha_{2}+\alpha_{5} S\right)\left(\alpha_{0}+\alpha_{3} S+\sum_{i=1}^{3} g^{i} e^{-h^{i} T^{i}}\right)}{\alpha_{0} \alpha_{6}+\alpha_{1}\left(\alpha_{2}+\alpha_{5} S\right)+S\left[\left(\alpha_{2} \alpha_{4}+\alpha_{0} \alpha_{7}+\alpha_{4} \alpha_{5} S+\alpha_{3}\left(\alpha_{6}+\alpha_{7} S\right)\right]+\left(\alpha_{6}+\alpha_{7} S\right) \sum_{j=1}^{3} g^{j} e^{-h^{j} T^{j}}\right.}, \\
& U^{3}=-\frac{\alpha_{0}+\alpha_{3} S+\sum_{i=1}^{3} g^{i} e^{-h^{i} T^{i}}}{\alpha_{2}+\alpha_{5} S} .
\end{aligned}
$$

We can now insert this solution back into Eq. (3.53). This way we derive the following effective superpotential:

$$
\begin{aligned}
& W_{\text {eff }}\left(S, T^{i}\right)=\left\{2 ( \alpha _ { 0 } + \alpha _ { 3 } S + \sum _ { i = 1 } ^ { 3 } g ^ { i } e ^ { - h ^ { i } T ^ { i } } ) \left[-\alpha_{1}\left(\alpha_{2}+\alpha_{5} S\right)+\alpha_{0}\left(\alpha_{6}+\alpha_{7} S\right)+i S\left(\alpha_{2} \alpha_{4}\right.\right.\right. \\
& \left.\left.\left.+\alpha_{4} \alpha_{5} S-\alpha_{3}\left(\alpha_{6}+\alpha_{7} S\right)\right)+\left(\alpha_{6}+\alpha_{7} S\right) \sum_{k=1}^{3} g^{k} e^{-h^{k} T^{k}}\right]\right\} /\left\{\sum_{j=1}^{3} g^{j} e^{-h^{j} T^{j}}\left(\alpha_{6}+\alpha_{7} S\right)\right. \\
& \left.\quad+\alpha_{0} \alpha_{6}+\alpha_{1}\left(\alpha_{2}+\alpha_{5} S\right)+S\left[\alpha_{2} \alpha_{4}+\alpha_{0} \alpha_{7}+\alpha_{4} \alpha_{5} S+\alpha_{3}\left(\alpha_{6}+\alpha_{7} S\right)\right]\right\}
\end{aligned}
$$

The numerator is a polynomial of third degree in $S$ and second degree in denominator. 
To apply the analysis of [35] requires to compute the ratio $\frac{S W_{\text {eff }}(S)^{\prime \prime}}{W_{\text {eff }}(S)^{\prime}}$ and to analyze, if its value is bigger than one. However, this analysis assumes a superpotential of the form $W_{\text {eff }}(S)+\sum_{i=1}^{3} g^{i} e^{-h^{i} T^{i}}$. Obviously, our effective superpotential (3.56) is not of this form. This would only be achieved for a special choice of the coefficients $\alpha_{i}$. The condition on $\alpha_{i}$ for the numerator and denominator be divisible without remainder is:

$$
\left(\alpha_{1}+\alpha_{4} S\right)\left(\alpha_{2}+\alpha_{5} S\right)=0
$$

After inserting this condition the effective superpotential 3.56) becomes

$$
W_{\mathrm{eff}}=2\left(\alpha_{0}+\alpha_{3} S+\sum_{i=1}^{3} g^{i} e^{-h^{i} T^{i}}\right)
$$

This is again the already analyzed case of the previous section, in which there is no stable minimum.

\subsection{Cubic superpotential}

We consider the case of three complex structure moduli $\left(U^{i}\right)$ and three Kähler moduli $\left(T^{i}\right)$. To make the calculations simple, we assume $U:=U^{1}=U^{2}=U^{3}, T:=T^{1}=T^{2}=$ $T^{3}$. The superpotential and the Kähler potential have the following form:

$$
\begin{gathered}
W=\alpha_{0}+\alpha_{1} U+\alpha_{2}(U)^{2}+\alpha_{3}(U)^{3}+S\left(\alpha_{4}+\alpha_{5} U+\alpha_{6}(U)^{2}+\alpha_{7}(U)^{3}\right)+3 g e^{-h T} \\
K=-\ln (S+\bar{S})-3 \ln (U+\bar{U})-3 \ln (T+\bar{T}), \quad g, \alpha_{i}, h \in \mathbf{R}, h \text { positive }
\end{gathered}
$$

We rewrite $U$ and $T$ using the real basis: $U=u+i \nu, T=t+i \tau$ and compute the supersymmetry conditions $\left(D_{U} W=D_{T} W=0\right)$ at the point of vanishing $\nu$ and $\tau$ :

$$
\begin{aligned}
& \left.\alpha_{0}=g e^{-h t}(-3+2 h t)+u\left(\alpha_{5} s+\alpha_{2} u+2 \alpha_{6} s u+2 \alpha_{3} u^{2}+3 \alpha_{7} s u^{2}\right)\right), \\
& \alpha_{1}=-\frac{1}{u} 3 g h t e^{-h t}-\alpha_{5} s-u\left(2 \alpha_{2}+2 \alpha_{6} s+3 \alpha_{3} u+\alpha_{7} s u\right), \\
& \alpha_{4}=-\frac{1}{s}\left(g h t e^{-h t}+s u\left(\alpha_{5}+\alpha_{6} u+\alpha_{7} u^{2}\right)\right) .
\end{aligned}
$$

As in the previous cases, we compute the scalar potential and its Hessian at the supersymmetric points. This means that we calculate the second derivatives of the potential and eliminate $\alpha_{0}, \alpha_{1}, \alpha_{4}$ by using (3.61). It is irrelevant which of the parameters or fields are eliminated through (3.61). We choose this particular combination by the criterion of simplicity of the later analysis. 
The Hessian should have positive eigenvalues at the minimum or equivalently, its upper-left submatrices should be positive definite. The determinants of the upper-left submatrices are of the form

$$
\begin{aligned}
a_{11}= & a_{44}=\frac{1}{48 s t^{3} u^{3}}\left(9 u^{4}\left(4 a_{3}^{2}+8 \alpha_{3} \alpha_{7} s+7 \alpha_{7}^{2} s^{2}\right)+u^{3}\left(24 \alpha_{2} \alpha_{3}+24 \alpha_{2} \alpha_{7} s+24 \alpha_{3} \alpha_{6} s+60 \alpha_{6} \alpha_{7} s^{2}\right)\right. \\
& \left.+u^{2}\left(4 \alpha_{2}^{2}+8 \alpha_{2} \alpha_{6} s+16 \alpha_{6}^{2} s^{2}+18 \alpha_{5} \alpha_{7} s^{2}\right)+12 \alpha_{5} \alpha_{6} s^{2} u+3 \alpha_{5}^{2} s^{2}\right)+\mathcal{O}\left(e^{-h t}\right) \\
a_{22}= & a_{55}=\frac{\left(\alpha_{5}+2 \alpha_{6} u+3 \alpha_{7} u^{2}\right)^{2}}{768 t^{4} u^{4}}+\mathcal{O}\left(e^{-h t}\right) \\
a_{33}= & \frac{g^{2} h^{2} e^{-2 h t}(2+h t)(1+2 h t)\left(\alpha_{5}+u\left(2 \alpha_{6}+3 \alpha_{7} u\right)\right)^{4}}{8192 s t^{9} u^{7}}+\mathcal{O}\left(e^{-3 h t}\right) \\
a_{66}= & \frac{g^{2} h^{3} e^{-2 h t}(3+2 h t)\left(\alpha_{5}+u\left(2 \alpha_{6}+3 \alpha_{7} u\right)\right)^{4}}{8192 s t^{8} u^{7}}+\mathcal{O}\left(e^{-3 h t}\right) .
\end{aligned}
$$

For positive flux parameters $\alpha_{2}, \alpha_{3}, \alpha_{5}, \alpha_{6}, \alpha_{7}$ and in the region of large $t\left(t^{-4}>>e^{-h t}\right)$, all sub-determinants are positive. So in the case of three complex structure moduli, there is some region for which there is a supersymmetric minimum.

\subsection{Open string moduli and soft-supersymmetry breaking terms}

So far, we have not yet discussed the open string moduli accounting for $D 3 / D 7-$ brane positions, Wilson line moduli and matter fields. The $D 7$-brane moduli $\phi_{7, j}$ are fixed through $W_{\text {flux }}$ in the case of $I S D$-fluxes with their respective scalars acquiring softsupersymmetry breaking masses $\widetilde{m}_{\phi_{7, j}}$. The latter have been calculated in [14 [17. Generically, the locations of the $D 7$-branes are fixed to those of the $O 7$-planes.

After including the non-perturbative superpotential $W_{\mathrm{np}}$, supersymmetry is restored at the minimum and the scalar masses take the generic form:

$$
\left(m_{\phi_{7, j}}\right)^{2}=-2 \kappa_{4}^{6} e^{\kappa_{4}^{2} \widehat{K}}|W|^{2} G_{\phi_{7, j} \bar{\phi}_{7, j}}=\frac{2}{3} \kappa_{4}^{4} V_{0} G_{\phi_{7, j} \bar{\phi}_{7, j}} .
$$

Here, $V_{0}$ is the value of the potential at the minimum (cf. Eq. (3.13) ) and $G_{\phi_{7, j} \bar{\phi}_{7, j}}$ is the metric of the fields $\phi_{7, j}$. Since supersymmetry is restored, these masses correspond to some sort of effective $\mu$-terms in the superpotential (3.1). Nevertheless, the soft-supersymmetry breaking mass terms $\widetilde{m}_{\phi_{7, j}}$ are important quantities as they represent certain coefficients of a low-energy supergravity expansion of the $D$-brane dynamics (encoded in the Born-Infeld action) coupled to non-vanishing 3-form fluxes. Moreover, as we shall argue in subsection 4.2, they are relevant for the discussion of possible non-perturbative contributions $W_{\mathrm{np}}$ to the superpotential. For the $\mathbf{Z}_{2} \times \mathbf{Z}_{2}$ orientifold, the relation between $m_{\phi_{7, j}}$ and $\widetilde{m}_{\phi_{7, j}}$ may 
be determined by the $F$-terms, given in subsection 4.1. E.g. the gaugino mass $m_{g, j}$ of a $D 7$-brane, which is transversal to the $j$-th internal complex 2 -plane becomes

$$
m_{g, j}=\widetilde{m}_{g, j}\left[1+\frac{\left(T^{j}+\bar{T}^{j}\right) g^{j} h^{j} e^{-h^{j} T^{j}}}{W_{\text {flux }}}\right],
$$

with $\widetilde{m}_{g, j}$ the gaugino mass before introducing the non-perturbative superpotential $W_{\mathrm{np}}$. Hence, e.g. for all Kähler moduli $T^{j}$ and the parameters $h^{j}, g^{j}$ equal, we find:

$$
\frac{m_{g, j}}{m_{g, k}}=\frac{\widetilde{m}_{g, j}}{\widetilde{m}_{g, k}} .
$$

Hence, the ratio of gaugino masses after inclusion of $W_{\mathrm{np}}$ is given by the same ratio as without $W_{\mathrm{np}}$. Strictly speaking, this ratio is only valid for the case $F^{M} \neq 0$. Similar relations may be found for other soft-masses. Eventually, soft-supersymmetry breaking terms after the KKLT uplift, i.e. after inclusion of the anti D3-branes, have been determined recently in Ref. [55].

The type $I I B$ orientifolds, introduced in section 2, imply 64 O3-branes with negative $D 3$-brane charges. At the orbifold point, no extra contribution comes from the induced D3-brane charge of wrapped D7/O7-stacks (without world-volume 2-form flux on the $D 7$ ). Hence tadpoles must be cancelled by adding $D 3$-branes or/and some amount of flux $N_{\text {flux }}$. The original KKLT proposal is, that only $I S D$-flux should be turned on in order to have a reliable supergravity approximation at least before the uplift. Since $I S D$-fluxes do not freeze the positions of $D 3$-branes, the tadpoles should be cancelled by pure flux, i.e. $N_{\text {flux }}=64$. It has been shown in Ref. [56], that $I S D$-fluxes with $N_{\text {flux }}=64$ are possible in the $\mathbf{Z}_{2} \times \mathbf{Z}_{2}$ orientifold.

\section{Non-perturbative superpotential, moduli stabilization and resolved orb- ifolds}

\subsection{The non-perturbative superpotential}

We will first present some general facts about the non-perturbative superpotential due to wrapped Euclidean D3-brane instantons. As an alternative but related mechanism to generate a non-perturbative superpotential we also discuss gaugino condensation in the effective $\mathrm{N}=1$ gauge theory that lives on $D 7$-branes wrapped on internal 4-cycles. As we will see, this possibility of getting a non-perturbative superpotential is largely influenced by the 3 -form flux turned on. Hence there is a very non-trivial interplay between the tree-level superpotential due to 3-form flux and the non-perturbative superpotential due to D3-brane instantons and/or gaugino condensation. Some useful papers on this subject include Refs. [24,57,25,26,27,28,32]. 
We start with some type $I I B$ orientifold on a 6-dimensional CY space $M_{3}$ with some possible 3 -form flux $G_{3}$ through some 3 -cycles $\Sigma$. Assume that $M_{3}$ possesses a complex 2-dimensional divisor, called $\mathcal{O}_{i}$. The relevant question is whether in $E q$. (3.1) nonperturbative effects contribute to a non-vanishing superpotential of the form

$$
W_{\mathrm{np}} \sim g_{i} e^{-h_{i} V_{i}},
$$

where $V_{i}$ denotes the volume of $\mathcal{O}_{i}$. The constant $h_{i}$ in the exponent depends on which mechanism, D3-brane instantons or gaugino condensation, is responsible for the generation of $W_{\mathrm{np}}$. The most important question is, if, for any given $\mathcal{O}_{i}, g_{i}$ is zero or not. (The even more difficult problem is what the value of $g_{i}$ is, and whether it depends on the complex structure moduli, which is expected on general grounds.) This question can be answered in three different, but related ways:

\section{(i) Euclidean D3-brane instantons in type IIB}

We consider possible contributions to $W_{\text {np }}$ from Euclidean $D 3$-branes wrapped on $\mathcal{O}_{i}$. In order to decide whether one gets a contribution to $W_{\mathrm{np}}$, one has to count the number of fermionic zero modes, $n_{f}$, on the world volume theory of the $D 3$-branes. According to [24], $n_{f}$ must be 2 in order for $g_{i}$ to be non-vanishing. The actual value for $n_{f}$ crucially depends on the number of supersymmetries which are supported by $\mathcal{O}_{i}$. However, this number also depends on the 3 -form flux $G_{3}$ turned on. Hence it is useful to define the number of fermionic zero modes in the presence of 3 -form flux by the number $n_{f}\left(G_{3}\right)$. The actual requirement for a non-vanishing superpotential now is that $n_{f}\left(G_{3}\right)=2$.

In a $N=1$ supersymmetric Yang-Mills gauge theory, the contribution from a single D3-instanton to the superpotential is given by the following expression:

$$
W_{\mathrm{np}} \sim \Lambda^{3 b} .
$$

$b$ is the $\beta$-function coefficient of the corresponding group (see below), and $\Lambda$ is the dynamical scale of the gauge theory:

$$
\Lambda^{3}=e^{-\frac{8 \pi^{2}}{b g^{2}}} .
$$

After relating the gauge coupling to the volume ( $c f$. subsection 4.3 for a definition of the gauge coupling) of $\mathcal{O}_{i}$,

$$
\frac{4 \pi}{g^{2}}=V_{i},
$$

one obtains

$$
W_{\mathrm{np}} \sim g_{i} e^{-2 \pi V_{i}} .
$$

So $h_{i}=2 \pi$. 
(ii) Euclidean M5-brane instantons in F-theory

A second description is given in terms of $F$-theory on a four-manifold $M_{4}$. Here we are considering Euclidean $M 5$-branes wrapped around a six-dimensional divisor $D_{i}$ inside $M_{4} . M_{4}$ is an elliptic $T^{2}$ fibration over some six-dimensional $I I B$ base $B_{6}$. The condition for a non-vanishing superpotential in $F$-theory now is given by the requirement that the arithmetic genus $\chi_{D_{i}}=1$ [58], where $\chi_{D_{i}}$ is given by the following formula:

$$
\chi_{D_{i}}=h_{(0,0)}\left(D_{i}\right)-h_{(0,1)}\left(D_{i}\right)+h_{(0,2)}\left(D_{i}\right)-h_{(0,3)}\left(D_{i}\right) .
$$

Just like in the D3-brane case, the number of fermionic zero modes, the arithmetic genus can be "changed" in the presence of 4 -form flux $G_{4}$ in $F$-theory. This gives rise to an effective arithmetic genus $\chi_{D_{i}}\left(G_{4}\right)$. Therefore it is not necessary that the geometric arithmetic genus $\chi_{D_{i}}$ is one, but rather the requirement for a non-vanishing superpotential becomes $\chi_{D_{i}}\left(G_{4}\right)=1$. Whether this condition is fulfilled depends again on the number of supersymmetries preserved by the flux. In particular, if the flux preserves $\mathrm{N}=2$ supersymmetry, then in general $\chi_{D_{i}}\left(G_{4}\right) \neq 1$. On the other hand if $G_{4}$ preserves $\mathrm{N}=1$, one can expect the above condition to be satisfied.

(iii) D7-branes in type IIB orientifolds and gaugino condensation

Instead of wrapping $D 3$-branes on $\mathcal{O}_{i}$ respectively 5 -branes on $D_{i}$, we may also consider a stack of $N D 7$-branes which fill the space-time and wrapped around four-dimensional divisors $\mathcal{O}_{i}$. In general orientifold compactifications, the existence of the $D 7$-branes is in fact forced by the tadpole cancellation conditions, namely in order to cancel the Ramond charge of the orientifold planes. Now consider the open string spectrum on the $D 7$-branes. It is in general given by an effective $\mathrm{N}=1$ supersymmetric $S U(N)$ gauge theory with some additional matter fields. In the following we will study the conditions, under which gaugino condensation will take place in the effective $\mathrm{N}=1$ gauge theory on the $D 7$-branes and will lead to a contribution to $W_{\mathrm{np}}$.

First consider pure $\mathrm{N}=1$ Yang-Mills gauge theory with gauge group $G$ without any matter fields. Gaugino condensation generates a nonperturbative superpotential

$$
W_{\mathrm{np}} \sim \Lambda^{3}=e^{-\frac{8 \pi^{2}}{b g^{2}}}
$$

With eq.(4.4) we then get

$$
W_{\mathrm{np}} \sim g_{i} e^{-\frac{2 \pi V_{i}}{b}}
$$

and hence $h_{i}=2 \pi / b$ for pure SQCD. 
Now consider N=1 SQCD with gauge group $G=S U\left(N_{C}\right)$ and with $N_{F}$ matter fields $Q, \widetilde{Q}$ in the fundamental plus anti-fundamental representations $N_{F}\left(\underline{N}_{C} \oplus \underline{N_{C}}\right)$. For $N_{F}<N_{C}$, there is a dynamically generated superpotential (for a review see e.g. [59])

$$
W_{\mathrm{np}}=\left(N_{c}-N_{f}\right)\left(\frac{\Lambda^{3 N_{C}-N_{F}}}{\operatorname{det} Q \widetilde{Q}}\right)^{1 /\left(N_{c}-N_{f}\right)} .
$$

Here, $b=3 N_{C}-N_{F}$ is the $\mathrm{N}=1 \beta$-function coefficient of SQCD. The vacuum expectation values of the meson superfields $M \sim Q \widetilde{Q}$ break the gauge group $S U\left(N_{C}\right)$ to the nonAbelian subgroup $S U\left(N_{C}-N_{F}\right)$. If $N_{F}=N_{C}-1$, the superpotential is generated by gauge instantons. On the other hand, the superpotential arises due to the gaugino condensation in the unbroken $S U\left(N_{C}-N_{F}\right)$ gauge group. Therefore the gaugino condensate is determined by the scale of the unbroken gauge group, $\langle\lambda \lambda\rangle \sim \Lambda_{N_{C}-N_{F}}^{3}$, where the scale $\Lambda_{N_{C}-N_{F}}$ of the low-energy $S U\left(N_{C}-N_{F}\right)$ gauge theory can be associated to the scale $\Lambda$ of the high-energy gauge theory as $\Lambda_{N_{C}-N_{F}}^{3\left(N_{C}-N_{F}\right)}=\Lambda^{3 N_{C}-N_{F}} / \operatorname{det} M$. This precisely yields the effective superpotential eq.(4.9). Finally, for $N_{F} \geq N_{C}$ there is no dynamically generated superpotential of this type.

It is also important to know what is happening if the squarks fields $Q$ and $\widetilde{Q}$ get a mass. Consider the case where we have $N_{F}^{\prime}$ matter fields with some common mass $m$. If we are interested in the effective low energy field theory, i.e. for energies much smaller than the masses of the squark fields, they decouple and the low energy theory is $S U\left(N_{C}\right)$ with $N_{F}-N_{F}^{\prime}$ flavors. For this theory the previous discussion applies. So in the case where all squark fields are heavy, one is back to gaugino condensation in pure SQCD.

An interesting class of relevant models are $\mathrm{N}=1$ quiver gauge theories with gauge group $G=S U\left(N_{C}\right) \times S U\left(N_{C}^{\prime}\right)$ and bifundamental matter fields in the representations $\left(\underline{N}_{C}, \overline{N_{C}^{\prime}}\right)+$ h.c. (plus possibly massless adjoint fields). These models generically appear as effective gauge theories of intersecting $D 7$-branes. One way to analyze the dynamics of these field theories is to consider $S U\left(N_{C}^{\prime}\right)$ as the flavor group of the $S U\left(N_{C}\right)$ gauge group and vice versa. Then the $\beta$-function coefficient for $S U\left(N_{C}\right)$ becomes $b=3 N_{C}-N_{C}^{\prime}$, and analogously for $S U\left(N_{C}^{\prime}\right)$. For $N_{C}^{\prime}>N_{C}$, gaugino condensation in the $S U\left(N_{C}\right)$ part is excluded, but is possible in the $S U\left(N_{C}^{\prime}\right)$ gauge theory. However, following our previous discussion, for $N_{C}^{\prime}=N_{C}$ it will not be possible in either of the two gauge group factors.

Next let us briefly discuss what will happen if one adds also $\widetilde{N}_{F}$ massless adjoint chiral matter fields $X$. The $\mathrm{N}=1 \beta$-function coefficient for $G=S U\left(N_{C}\right)$ becomes $b=$ $\left(3-\widetilde{N}_{F}\right) N_{C}-N_{F}$. Obviously, for $\widetilde{N}_{F} \geq 3$ asymptotic freedom is lost, and there will be no non-perturbative superpotential. However also the remaining cases $\widetilde{N}_{F}=1,2$ are problematic. For $\widetilde{N}_{F}=1$ and $N_{F}=0$ without tree level superpotential, the theory becomes $\mathrm{N}=2$ supersymmetric. This theory is known to possess a moduli space in the Coulomb branch without dynamical superpotential. Adding a tree level superpotential 
$W_{\text {tree }} \sim \operatorname{Tr} X^{k}+\ldots$ might change this conclusion, but the discussion now becomes more model dependent. Similarly for $\widetilde{N}_{F}=2$ with a tree level superpotential it is not possible to make very specific statements.

In conclusion we have seen that the possibility of having a non-perturbative superpotential is indeed rather model dependent. Therefore we like to simplify the discussion and want to formulate a few simple conditions, which are sufficient but not always necessary for a non-perturbative superpotential (later we will discuss how these rules can by implemented by the $D 7$-branes in orientifold models):

1.) For a non-vanishing $W_{\mathrm{np}}$ we require that all fundamental matter fields, if present, become massive. This conditions implies some strong conditions on the world volume theory of the D7-branes. Massless bi-fundamental matter fields typically arise as open string states localized at the intersection loci of two D7-branes. The number of those states depends on the geometrical intersection number and also on the open string 2 -form magnetic fluxes on the $D 7$-branes. So we will essentially require that those $D 7$-branes which are responsible for gaugino condensation in the hidden gauge sectors do not intersect each other.

2.) For a non-vanishing $W_{\mathrm{np}}$ we require that all adjoint matter fields, if present, become massive. As we will discuss later, the number of massless adjoint chiral multiplets is a 3-form flux dependent quantity. This means in particular, that possibly after turning on some 3-form flux,

a) there are no massless adjoint scalar fields associated to the positions of the D7-branes in the space transversal to $\mathcal{O}_{i}$ (called position fields $\phi_{7}$ in the following),

b) there are no massless adjoint scalar fields living on the $D 7$-branes being associated to Wilson lines (called Wilson line fields $\phi_{7}^{\prime}$ in the following).

\section{2. $M_{3}=T^{6} /\left(\mathbf{Z}_{N} \times \mathbf{Z}_{M}\right)$}

These are the models which are of main interest in our paper. The simplest model of this class is the $\mathbf{Z}_{2}$ orientifold with $M_{3}=T^{6}$. This model was recently considered in [26] and some time ago originally in [11]. In this case, the $F$-theory lift is described by $M_{4}=\left(T^{4} \times K 3_{2}\right) / Z_{2}$. All type $I I B$ divisors are simply $T^{4}$. In $F$-theory, we get $D_{i}=T^{4} \times T^{2}$. Since $\chi_{T^{4} \times T^{2}}=0$, a non-perturbative superpotential due to Euclidean 5 -branes is excluded. We briefly compare this result with the spectrum of the type $I I B$ 
orientifold. Since $T^{4}$ supports 16 supercharges, it simply follows that $n_{f}=16$, i.e. no superpotential.5

The spectrum can be again changed by turning on 3-form flux. As shown in [26], the number of fermionic zero modes on the $D 3$-branes is reduced by a particular $(2,1)$ 3 -form flux $G_{2,1}$ from 16 to $n_{f}\left(G_{3}\right)=4$. So we see that turning on $G_{2,1}$ is not sufficient to generate $W_{\mathrm{np}}$. However, one expects that by turning on additional 3-form fluxes, the number of fermionic zero modes is further reduced, and a non-perturbative superpotential may eventually become possible.

Now we will turn to the general $T^{6} /\left(\mathbf{Z}_{N} \times \mathbf{Z}_{M}\right)$ orientifolds. Many of these orientifolds require $D 7$-branes for tadpole cancellation. Since the $\mathbf{Z}_{N} \times \mathbf{Z}_{M}$ orbifold group now has a non-trivial action on the six-dimensional space, the four-dimensional divisors $\mathcal{O}_{i}$ split into two classes: (a) untwisted divisors $D_{i}$ and (b) twisted, i.e. exceptional divisors $E_{\alpha}$. The untwisted divisors are in one-to-one correspondence with the untwisted 4-cycles of the orbifold. The number of linearly independent untwisted divisors is precisely given by the untwisted Hodge number $h_{(1,1)}^{\text {untw. }}$ of the orbifold, i.e. the number of untwisted Kähler moduli. On the other hand, the twisted divisors correspond to the twisted, blown-up 4-cycles of the orbifolds, which arise after resolving the orbifold singularities. Hence the number of linearly independent twisted divisors is given by the number of twisted Kähler moduli on the orbifold, namely by $h_{(1,1)}^{\text {twist. }}$. The actual values for $h_{(1,1)}^{\text {untw. }}, h_{(1,1)}^{\text {twist. in each }}$ of the orientifold examples can be found in Table 1 and 2. According to our previous discussion, a gaugino condensate will form if there are no massless fundamental and no massless adjoint matter fields on the world volume theories of the $D 7$-branes. This means that the respective divisors do not intersect each other and hence there are no massless bifundamental fields, and that the adjoint matter fields are absent or massive due to 3-form fluxes. In the following we will now consider each case separately:

\section{(a) Untwisted divisors in the orbifold limit}

In the unresolved orbifolds, the untwisted divisors always have topology $D_{i} \sim T^{4}$. The untwisted open string states on the $D 7$-branes wrapped on $T^{4}$ are always given by $\mathrm{N}=4$ super Yang-Mills multiplets of the gauge group $G$. It contains 1 adjoint chiral position field $\phi_{7}$ and 2 adjoint Wilson line fields $\phi_{7}^{\prime}$. In addition, if we consider $N_{C}^{i}$ and $N_{C}^{j} D 7$-branes

5 It is perhaps interesting to note that the geometric arithmetic genus of $T^{4} \times T^{2}$, expressed in terms of the Hodge numbers of $T^{4}$ can be written as

$$
\chi_{T^{4} \times T^{2}}=h_{(0,0)}\left(T^{4}\right)-h_{(0,1)}\left(T^{4}\right)+h_{(0,2)}\left(T^{4}\right)=0 .
$$

The actual numbers $h_{(0,1)}\left(T^{4}\right)=2$ and $h_{(0,2)}\left(T^{4}\right)=1$ perfectly agree with the number of massless adjoint fields on the $D 7$-branes. 
wrapped on two untwisted divisors $D_{i}$ and $D_{j}$, there are massless bi-fundamental matter fields. More specifically, the three $D_{i}$ are defined by the algebraic conditions $z^{i}=a^{i}=$ const. Therefore they mutually intersect each other on the (complex) one-dimensional subspaces $z^{i}=a^{i}, z^{j}=a^{j}(i \neq j)$. The corresponding triple intersection number is just one. (Here we do not consider possible 2 -form flux on the $D 7$-branes.) The twisted open string spectrum on this common intersection locus can be easily determined and is given by massless bi-fundamental matter fields in the representations $\left(\underline{N}_{C}^{i}, \overline{N_{C}^{j}}\right)+$ h.c. of the gauge group $G=U\left(N_{C}^{i}\right) \times U\left(N_{C}^{j}\right)$. Similarly, if we consider models with more than three Kähler moduli, like the $\mathbf{Z}_{3}$ and $\mathbf{Z}_{6-I}$ orientifolds, the additional untwisted divisors are defined by equations like $D_{4}=z_{1}+z_{2}=$ const. These divisors mutually intersect with all other $D_{i}$. (However the triple intersection number vanishes for some combinations, e.g. the triple intersection number $D_{1} \cdot D_{2} \cdot D_{4}=0$ ). Therefore we again get massless bi-fundamental matter fields on the intersection of all these untwisted divisors.

So the presence of the massless adjoint fields $\phi_{7}$ and $\phi_{7}^{\prime}$ and also the possible bifundamental matter fields due to D7-brane intersections will hamper the formation of a gaugino condensate. Hence the important question is again, which 3-form fluxes make the fields $\phi_{7}$ massive, and which 3-form fluxes are responsible for the masses of the fields $\phi_{7}^{\prime}$. More precisely, in order to get a non-vanishing superpotential, those 3 -form fluxes have to be turned on in such a way that the $\mathrm{N}=4$ supersymmetry on the world volume of the $D 7$-branes is broken to $\mathrm{N}=1$ supersymmetry. Then the untwisted open string spectrum contains only one massless adjoint $\mathrm{N}=1$ vector multiplet and no further adjoint chiral multiplets. This should be possible by turning on two kinds of possible 3-form fluxes. First, $G_{2,1}$ generically gives a mass to $\phi_{7}$. This is the effective, supersymmetric $\mu$-term which is of the following form [18]:

$$
\mu_{\phi_{7} \phi_{7}} \sim \int \bar{G}_{3} \wedge \omega_{A}
$$

Next consider 3-form fluxes which can give a mass also to the Wilson line fields. This kind of 3-form fluxes were considered in [14] and in [16] in the context of softly supersymmetry breaking mass parameters. There, it was shown that the $(0,3)$-form flux $G_{0,3}$ indeed generates a mass for the adjoint Wilson line scalar fields $\phi_{7}^{\prime}$. (In addition, the $(3,0)$-form flux $G_{3,0}$ flux will also contribute to the masses of the fields $\phi_{7}$.) The specific mass term for the two adjoint matter fields has following form [14,16] (in case of vanishing 2-form flux on the $D 7$-branes):

$$
\left(m_{\phi_{7}^{\prime} \phi_{7}^{\prime}}^{1}\right)^{2},\left(m_{\phi_{7}^{\prime} \phi_{7}^{\prime}}^{2}\right)^{2} \sim\left|\int \bar{G}_{3} \wedge \Omega\right|^{2} .
$$

More precisely, at the extremum $V_{0}$ of the scalar potential, the soft-masses are given by $E q$. (3.63). One might worry that $G_{(0,3)}$ completely breaks all supersymmetries on the 
$D 7$-branes. This is indeed the case without $W_{\mathrm{np}}$. However, as we have seen in the previous chapter, in the context of full moduli stabilization with non-perturbative superpotential, supersymmetric AdS-vacua are possible in the presence of these more general 3-form fluxes. So in conclusion, without being more specific, it is quite reasonable to assume that after turning on specific 3 -form flux components $G_{(0,3)}, G_{(3,0)}$ and, if allowed by the orbifold twist, also $G_{(2,1)}, G_{(1,2)}$, all unwanted adjoint matter fields become massive.

Next we turn to the problem of the massless matter fields in bi-fundamental representations due to D7-brane intersections. As explained before, they are always present if more than one stack of $D 7$-branes are wrapped on the untwisted divisors $D_{i}$ in the unresolved orbifold space. However after the resolution of the orbifold singularities some of the divisors $D_{i}$ do not intersect anymore. In physical terms this means that the twisted blowing-up modulus fields acts as a mass parameter for the bi-fundamental matter fields. Therefore the bi-fundamental matter fields from open strings spanned between $D 7$-branes around these two divisors became massive. A short discussion on resolved orbifolds will be given in section (4.3). Many more details based on a toric description of the divisors will be presented in [31].

\section{(b) Twisted divisors}

The twisted divisors $E_{\alpha}$ correspond to the twisted 4-cycles on the orientifold and receive a finite volume after blowing up the orbifold singularities. Generically they cannot be moved inside the compact space. The orbifold group generically reduces the number of supersymmetries from $\mathrm{N}=4$ down to $\mathrm{N}=1$. Therefore the twisted divisors are expected to contribute to the superpotential without turning on additional 3 -form fluxes. In $F$-theory language it means that the arithmetic genus of the twisted divisors is generically equal to one. This can be also seen from the D7-brane perspective. Wrapping D7-branes around twisted divisors means that we are considering open strings at orbifold singularities. After blowing up the twisted 4-cycles, the corresponding open string gauge theory on the D7branes generically is that of pure $\mathrm{N}=1$ Yang-Mills. Hence gaugino condensation will take place. If one wants to avoid massless chiral multiplets in the bi-fundamental representation of the gauge group, one has to choose disjoint twisted divisors which do not intersect. The intersection pattern of the $E_{\alpha}$ can be again best read off from the toric diagrams, cf. Ref. [31]. It can be seen that there exist several possibilities for twisted divisors $E_{\alpha}$ that do not mutually intersect.

As a specific model with exceptional divisors that lead to a non-vanishing superpotential the $T^{6} /\left(\mathbf{Z}_{2} \times \mathbf{Z}_{2}\right)$ orientifold was recently investigated in full detail in 32]. This model allows for an $F$-theory description in terms of an elliptic Calabi-Yau four-fold. The superpotential arises due to 48 D3-branes which are wrapped around all 48 linearly independent exceptional divisors. In addition, this model also contains 12 stacks of 8 D7-branes which are wrapped on 12 disjoint divisors that are linear combinations of the 3 untwisted divisors 
and 12 particular twisted divisors. In this model, the $D 7$-branes cancel the Ramond charge of the 12 orientifold O7-planes in a local way. It follows that the open string spectrum is given by pure $\mathrm{N}=1$ Yang-Mills with gauge group $G=S O(8)^{12}$.

Let us try to summarize the discussion of this section: in $\mathrm{N}=1$ orientifolds, Euclidean D3-instantons and/or gaugino condensation on D7-branes generically do generate a nonperturbative superpotential. For $D 3 / D 7$-branes being wrapped around untwisted divisors one needs certain 3-form fluxes for a non-vanishing superpotential. Moreover if one wants to avoid massless matter fields in bi-fundamental representations due to $D$-brane intersections, one has to resolve some or all orbifold singularities. If the branes are wrapped around twisted divisors, all conditions for a non-vanishing superpotential are in general met without any further fluxes. We are confident that the conditions for a non-vanishing $W_{\text {np }}$ can be satisfied in many or if not all of the considered orientifold spaces, although we do not provide here concrete, full fledged string models with all tadpole conditions satisfied. (An nice concrete example is the model considered in [32].) Nevertheless we assume that the total non-perturbative superpotential can be written in the following form:

$$
W_{\mathrm{np}}=\sum_{j=1}^{n_{3}^{\text {untw. }}} e^{-2 \pi V_{j}^{\text {untw. }}}+\sum_{\alpha=1}^{n_{3}^{\text {twist. }}} e^{-2 \pi V_{\alpha}^{\text {twist. }}}+\sum_{j^{\prime}=1}^{n_{7}^{\text {untw. }}} e^{-\frac{2 \pi V_{j^{\prime}}^{\text {untw. }}}{b_{j^{\prime}}(G)}}+\sum_{\alpha^{\prime}=1}^{n_{7}^{\text {twist. }}} e^{-\frac{2 \pi V_{\alpha^{\prime}}^{\text {twist. }}}{b_{\alpha^{\prime}}(G)}} .
$$

The first two terms denote the non-vanishing contributions of the Euclidean $D 3$-instantons

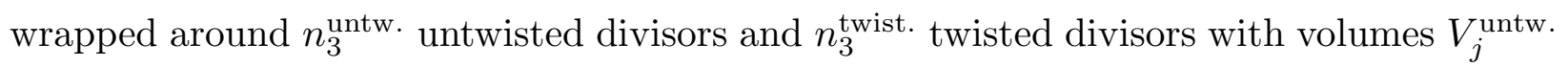
or $V_{\alpha}^{\text {twist. }}$, respectively. The last two terms describe the contribution from the gaugino condensation on different stacks of $D 7$-branes, which are wrapped around $n_{7}^{\text {untw. untwisted }}$

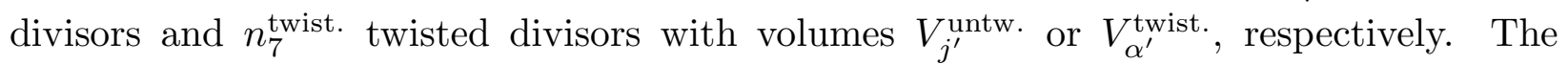
numbers $b_{j^{\prime}}(G), b_{\alpha^{\prime}}(G)$ are the suitably normalized $\beta$-function coefficients of the associated effective gauge theories. For pure $\mathrm{N}=1$ Yang-Mills, the $\beta$-functions coefficients are just given by the quadratic Casimir invariants $c_{2}(G)$ of the gauge group $G$. It is instructive to compare this superpotential with the one obtained in the $\mathbf{Z}_{2} \times \mathbf{Z}_{2}$ model of [32]. Here, there are 48 twisted divisors contributing to the D3-brane part and 12 twisted divisors associated to the $D 7$-branes; setting all twisted volumes $V_{\alpha}^{\text {twist. }}$ and also all volumes $V_{\alpha^{\prime}}^{\text {twist. equal to }}$ each other, the superpotential (4.13) reduces to (see eq.(6.17) in [32])

$$
W_{\mathrm{np}}=48 e^{-2 \pi V_{\alpha}^{\mathrm{twist}}}+12 e^{-\frac{2 \pi V_{\alpha^{\prime}}^{\mathrm{tw} \text { ist. }}}{6}} .
$$

\subsection{Moduli Stabilization in the resolved $\mathbf{Z}_{2} \times \mathbf{Z}_{2}$ orientifold revisited}

It has been pointed out in Ref. [35] and demonstrated in detail in subsection 3.6 for the case $h_{(1,1)}^{\text {untw. }}=3, h_{(2,1)}^{\text {untw. }}=1$, that first integrating out the dilaton field $S$ and the complex structure moduli $U^{j}$ is not necessary to find a stable supersymmetric minimum 
w.r.t. the Kähler moduli $T^{i}$. In fact, in the recent work 32, it has been shown that a stable supersymmetric minimum is possible in the resolved $\left(T^{2}\right)^{3} / \mathbf{Z}_{2} \times \mathbf{Z}_{2}$ orientifold. More concretely, the latter type $I I B$ compactification with $h_{(1,1)}=51, h_{(2,1)}=3$ allows for supersymmetric minima with all moduli fixed at the same time, i.e. without integrating out first $U^{j}$ and $S$. This result is interesting as the Kähler potential involves a non-trivial dependence on the Kähler moduli which is quite different from the cases discussed in section 3. Moreover, both $I S D$ and $I A S D$ fluxes are turned on.

In this subsection, we shall investigate some aspects of the stabilization of [32]. With $r_{i}$ being the volume of the three two-tori $T^{2, i}$ and $t_{i \alpha, j \beta}$ the size of the 48 exceptional divisors, the Kähler potential for the Kähler moduli reads [32]:

$$
K=-2 \ln (V)=-2 \ln \left(r^{3}-24 r t^{2}+48 t^{3}\right)
$$

on the locus of moduli space, which maximally respects the symmetries between the divisors, i.e. $r=r_{i}, t_{i \alpha, j \beta}=t$. The parameters $r, t$ refer to the string moduli fields (cf. the discussion in subsection 2.5). Hence, with $\operatorname{Re} T^{1}=e^{-\phi_{10}}\left(\frac{1}{3} \frac{\partial V}{\partial r}+\frac{1}{6} \frac{\partial V}{\partial t}\right)$ and $\operatorname{Re} T^{2}=-\frac{1}{48} e^{-\phi_{10}} \frac{\partial V}{\partial t}$ following from the relation (2.8), we determine the field-theoretical moduli to be:

$$
T^{1}=e^{-\phi_{10}}\left(r^{2}-8 r t+16 t^{2}\right)+i \int_{C_{1}} C_{4} \quad, \quad T^{2}=e^{-\phi_{10}}\left(r t-3 t^{2}\right)+i \int_{C_{2}} C_{4} .
$$

This allows us to express the Kähler potential (4.15) with the holomorphic coordinates:

$$
\begin{aligned}
K_{K} & =6 \ln 2+2 \ln \left(T^{2}+\bar{T}^{2}\right)-\ln \left\{\left(T^{1}+\bar{T}^{1}\right)+2\left(T^{2}+\bar{T}^{2}\right)\right. \\
& \left.-\left(T^{1}+\bar{T}^{1}\right)^{1 / 2}\left[T^{1}+\bar{T}^{1}+4\left(T^{2}+\bar{T}^{2}\right)\right]^{1 / 2}\right\} \\
& -2 \ln \left\{\left(T^{1}+\bar{T}^{1}\right)^{2}+16\left(T^{1}+\bar{T}^{1}\right)\left(T^{2}+\bar{T}^{2}\right)+32\left(T^{2}+\bar{T}^{2}\right)^{2}\right. \\
& +\left(T^{1}+\bar{T}^{1}\right)^{3 / 2}\left[\left(T^{1}+\bar{T}^{1}\right)+4\left(T^{2}+\bar{T}^{2}\right)\right]^{1 / 2} \\
& \left.+8\left(T^{2}+\bar{T}^{2}\right)\left(T^{1}+\bar{T}^{1}\right)^{1 / 2}\left[\left(T^{1}+\bar{T}^{1}\right)+4\left(T^{2}+\bar{T}^{2}\right)\right]^{1 / 2}\right\} .
\end{aligned}
$$

The Kähler potential for the dilaton and three (untwisted) complex structure moduli $U^{j}$, with $U^{j}=U$, is given by (cf. section 2 )

$$
K_{C S}=-\ln (S+\bar{S})-3 \ln (U+\bar{U})
$$

With these Kähler potentials, the tree-level superpotential $W_{\text {flux }}\left(S, U^{j}\right)$ of (3.1) and the non-perturbative superpotential (4.14), with $V_{\alpha}^{\text {twist. }}=T^{2}$ and $V_{\alpha^{\prime}}^{\text {twist. }}=T^{1}$, i.e. the full superpotential

$$
W=W_{\text {flux }}\left(S, U^{j}\right)+12 \Lambda e^{-2 \pi \frac{T^{1}}{6}}+48 \Lambda e^{-2 \pi T^{2}}
$$


a miminum is found at the values

$$
T^{1}=5.84, T^{2}=1.18, U=0.84+0.46 i \quad, \quad S=3.64+7 i,
$$

with $-\Lambda=e^{-K_{C S} / 2}=5.87$. Note, that the factor $\Lambda$ has been introduced 6 in [32] as "Kähler gauge". Though the fluxes turned on are non-supersymmetric, supersymmetry is restored after including the non-perturbative potential (4.14) and all moduli are stabilized at the above values. Indeed, we have checked that the mass matrix for the scalar fields $S, U, T^{1}, T^{2}$ is positive definite, with the following eight positive eigenvalues:

$$
\begin{array}{r}
\{0.00010817,0.000128819,0.00030082,0.000354985 \\
0.0105129,0.0112272,0.0925661,0.096739\}
\end{array}
$$

Like the orbifold example discussed in subsection 3.6, this represents a case where the combined minimization of the moduli $S, U, T^{1}, T^{2}$ at one stroke leads to a stable minimum. As one can see from the eigenvalues, the masses of the scalars of the dilaton and complex structure moduli are by a factor of one hundred larger than those of the Kähler moduli.

The Kähler potentials for the blown up orbifold compactifications (e.g. Eq. (4.17) for $\mathbf{Z}_{2} \times \mathbf{Z}_{2}$ in the symmetric resolution) take a quite different form from the ones for the cosets in (2.7), relevant to the unresolved orbifolds. Hence the discussion about finding stable minima in the context of orbifold limits of orientifolds in section 3 has to be redone for those more involved Kähler potentials [31].

One may now ask the question whether with a more complicated Kähler potential such as (4.17), first integrating out the complex structure modulus is possible, i.e. whether a minimum may still be found. To check this, let us start with the superpotential

$$
W=\alpha_{1}+\alpha_{2} S+12 e^{-2 \pi \frac{T^{1}}{6}}+48 e^{-2 \pi T^{2}}, \alpha_{i} \in \mathbf{C}
$$

and the Kähler potential (4.17) for the Kähler moduli and $K_{S}=-\ln (S+\bar{S})$ for the dilaton field. This may be a situation which appears after integrating out one complex structure modulus or it may mimic the situation of resolved orbifolds without complex structure moduli. To minimize the potential, we proceed like in the previous section: At the extremum for which $F_{S}, F_{T^{1}}, F_{T^{2}}=0$, we solve for the parameters $\alpha_{1}$ and $\alpha_{2}$, obtaining a non-trivial relation between the parameters $\alpha_{1}, \alpha_{2}$ and the moduli $S, T^{1}, T^{2}$. However, after plugging these relations into the mass matrix, we find that the latter has negative

6 In fact, in practice [32], one may first look for a minimum in the $T^{1}, T^{2}$-sector for constant $S, U^{j}$ and $W_{\text {flux }}\left(S, U^{j}\right)$, then promote this minimum to a minimum in the full moduli space $S, U, T^{1}, T^{2}$ through introducing the Kähler gauge. Of course, the values for $T^{1}, T^{2}$ do not change this way. 
eigenvalues. Hence, there is in general no minimum for this setup with one complex structure modulus integrated out, though stable minima may be found within the Kähler moduli sector $T^{1}, T^{2}$, while keeping $S$ fixed ( $c f$. the previous footnote). On the other hand to discuss the situation in the above discussed $\mathbf{Z}_{2} \times \mathbf{Z}_{2}$ orientifold one has to integrate out three complex structure moduli. The results, discussed in subsection 3.7, should be combined with the above non-perturbative superpotential.

To conclude, while for orbifolds with complex structure moduli it is always possible to find a stable minimum both at the orbifold point and after resolving, orbifolds without complex structure moduli do not seem to allow for moduli stabilization both at the orbifold point and after their resolution. This issue among others will be discussed in more detail in 31 .

\subsection{Outlook: Resolved Orbifolds}

As the analysis in section 3 has shown, all the those orbifolds with no complex structure moduli do not allow a stable vacuum. This leaves only the $\mathbf{Z}_{6-I I^{-}}$orbifolds on the different lattices, the $\mathbf{Z}_{2} \times \mathbf{Z}_{2^{-}}$and the $\mathbf{Z}_{2} \times \mathbf{Z}_{6}$-orbifold in the game. But can these receive contributions to the non-perturbative superpotential at the orbifold point?

From D3-brane instantons, it must be checked whether any of the divisors present fulfill the criterion $\chi=1$. Exceptional divisors, resulting from blowing up the orbifold singularities are likely to contribute, as they are fixed at the locations of the singularities and therefore have no moduli.

Let us now consider contributions originating from gaugino-condensates. $\mathbf{Z}_{2} \times \mathbf{Z}_{2}$ and $\mathbf{Z}_{2} \times \mathbf{Z}_{6}$ have each 12 O7-planes using the standard orientifold involution, sitting at the planes corresponding to $z^{i}=0, \frac{1}{2}, \frac{1}{2} \tau, \frac{1}{2}(1+\tau), z^{j}, z^{k}=$ free, where $\tau$ is the modular parameter of the torus corresponding to $z^{i}$. Canceling the tadpoles locally by placing the D7-branes on top of the O7-planes, we end up having bifundamental matter, as the divisors wrapped by the $D 7$-branes intersect. This means that we do not have a pure SYM-theory and therefore no contribution to the non-perturbative superpotential can arise. Resolving the singularities via blow-ups cures this problem, as the arising exceptional divisors change the intersection pattern of the divisors such that the divisors which are wrapped by the $D 7-$ branes no longer intersect. That we get gaugino condensates for $\mathbf{Z}_{2} \times \mathbf{Z}_{2}$ has been shown in [32]. For $\mathbf{Z}_{2} \times \mathbf{Z}_{6}$, the question whether there is adjoint matter must be settled first. The $\mathbf{Z}_{6-I I^{-}}$orbifolds have four stacks of $O 7$-planes with the exception of the $S U(2) \times S U(6)-$ lattice which has one stack of $O 7-$ planes. For these cases, the question of existence of gaugino condensates at the orbifold point remains to be studied in detail.

When resolving the orbifold singularities, all orbifolds except for the prime orbifolds $\mathbf{Z}_{3}, \mathbf{Z}_{7}$ and $\mathbf{Z}_{3} \times \mathbf{Z}_{3}$ which do not allow 07 -planes are possible candidates for moduli stabilization via gaugino-condensates. It is more difficult to settle the case for contributions 
coming from $D 3$-brane instantons, as an analysis of the $F$-theory lift of the model seems inevitable, yet for many of the orbifolds in question, no $F$-theory lift is known to exist. At any rate, orbifold models without complex structure moduli do not exhibit a stable uplift even in the resolved case [31].

We will address the questions outlined above in Ref. [31]. The first steps will be the local analysis of the models at the location of the orbifold singularities via the methods of toric geometry. In a next step, the local patches must be glued together to form the compact model. An orientifold-action must be determined, and after the divisors which are wrapped by the $D 7$-branes have been identified and their topology analyzed, it is possible to ascertain the existence of gaugino-condensates.

As an illustration, we present here the toric diagrams of the blow-ups of the local models for $\mathbf{Z}_{6-I I}$ and $\mathbf{Z}_{2} \times \mathbf{Z}_{6}$. Near the location of a fixed point, the orbifold $T^{6} / \mathbf{Z}_{N}$ looks like $\mathbf{C}^{3} / \mathbf{Z}_{N}$, irrespective of the lattice the orbifold lives on. The lattice becomes important when the local patches are glued together to form the compact model, as for each lattice, the configuration of the singularities turns out to be different.
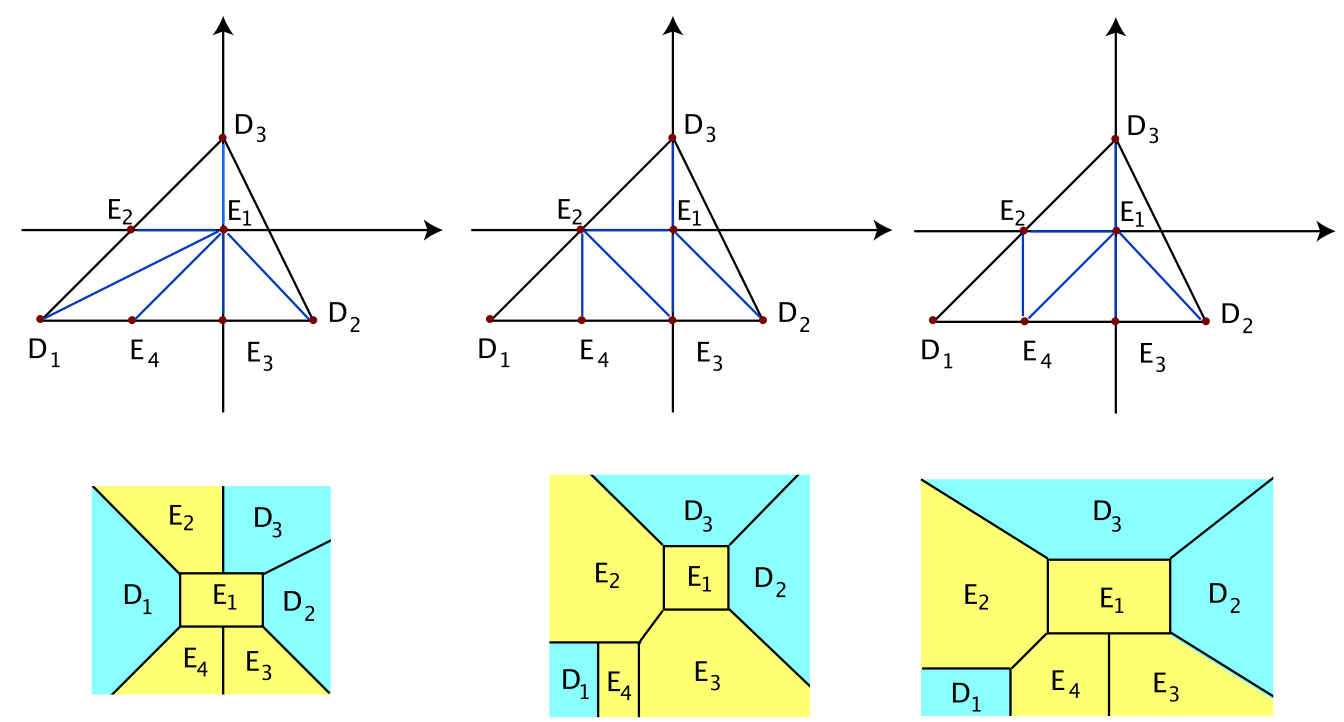

Figure 1: Toric diagrams of the resolutions of $\mathbf{C}^{3} / \mathbf{Z}_{6-I I}$ and dual graphs

Figure 1 shows three of the five possible resolutions of $\mathbf{C}^{3} / \mathbf{Z}_{6-I I}$. The other two can be obtained by taking case b) and flopping the curve $E_{1} \cdot E_{2}$ to $E_{3} \cdot D_{2}$ and taking case c) and flopping the curve $E_{1} \cdot E_{3}$ to $D_{3} \cdot E_{4}$. The $D_{i}$ denote the divisors inherited from the unresolved geometry, whereas the $E_{i}$ denote the exceptional divisors. There is one 
compact exceptional divisor, namely $E_{1}$. The diagrams in the bottom row are the dual graphs, where faces have become vertices and vice versa.

Figure 2 shows one of the 156 possible resolutions 0 of $\mathbf{C}^{3} / \mathbf{Z}_{2} \times \mathbf{Z}_{6}$. Here, we have two compact exceptional divisors, namely $E_{7}$ and $E_{8}$.
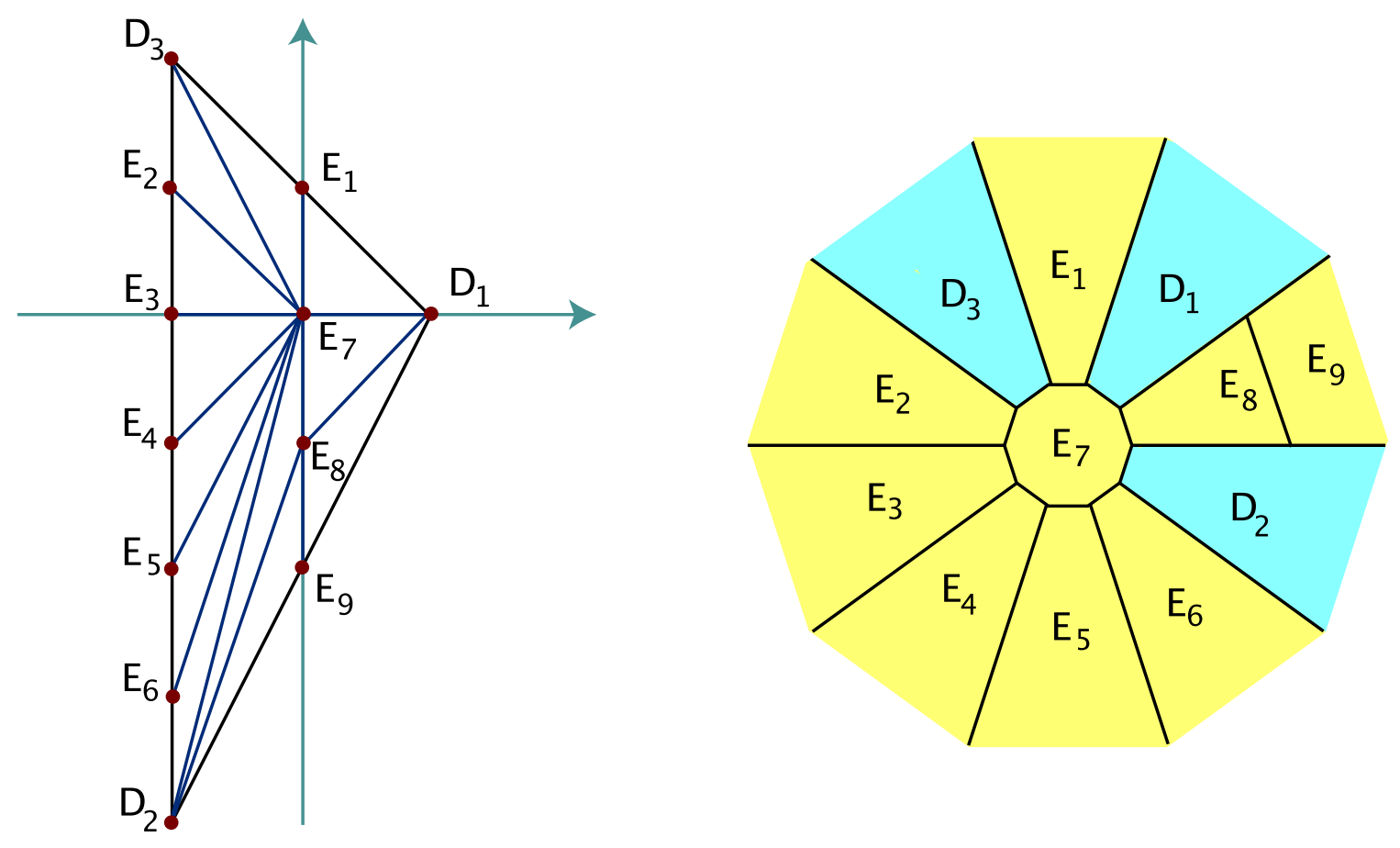

Figure 2: $\quad$ Toric diagram of one of the resolutions of $\mathbf{C}^{3} / \mathbf{Z}_{2} \times \mathbf{Z}_{6}$ and dual graph

\section{Conclusions}

This paper deals with moduli stabilization in type $I I B$ orientifolds à la KKLT, i.e. with tree-level 3-form flux superpotential plus non-perturbative superpotential from $D 3$ instantons and/or gaugino condensation. The main emphasis of the work are orientifold compactifications in their various orbifold limits. We show that it is indeed possible to find stable (i.e. tachyon free), supersymmetric AdS-minima with stabilized dilaton, untwisted Kähler $T^{i}$ and untwisted complex structure moduli $U^{j}$, as long as the geometrical orbifold group still allows for the existence of untwisted complex structure moduli fields. On the other hand, if the orbifold group action already freezes all complex structure moduli, then the scalar potential in the $S, T^{i}$-sector is such that the (mass) ${ }^{2}$ matrix for these fields contains negative eigenvalues. We also point out some problems with the integrating out procedure of complex structure moduli, namely we investigate cases, where integrating

7 The number of possible triangulations was obtained using the package TOPCOM. 
out the $U^{j}$ leads to non-stable AdS-vacua in the remaining $S, T^{i}$ potential, whereas the minimization of the full $S, T^{i}, U^{j}$ potential does not suffer from any instabilities.

In the paper we give a complete classification of the untwisted moduli spaces of all type $I I B$ orientifolds, together with the flux quantizations of the Ramond and NS 3-form fluxes on the orbifold spaces. We also provide the correct definitions of the untwisted Kähler moduli fields for type IIB orientifolds, including also the orbifolds with more than three Kähler moduli $T^{i}$. However we would like to emphasize that we do not claim in this paper that we have constructed full-fledged type $I I B$ orientifold models with all moduli fixed. The reasoning for this caveat is two-fold:

(i) We mainly stick to the orbifold limit. Hence we do not analyze the stability properties of the blow-up (i.e. twisted) moduli, which are nevertheless still present.

(ii) We do not discuss in great detail the form and the microscopic origin of the nonperturbative superpotential, but just parameterize it by a sum of exponential functions given in terms of the untwisted Kähler moduli. Again, the blowing up of the orbifold singularities will be very important for a more complete analysis.

Both these points as well as other aspects will be addressed in a forthcoming paper [31]. In this work we just remark on some properties of the potential including blowing-up moduli of the concrete type orientifold, which was recently constructed in [32]. Specifically, we extend the work of [32 by writing the Kähler potential and the superpotential in terms of the correct supergravity variables for the Kähler moduli fields. In this way, a Kähler potential is obtained which differs quite a bit from the standard Kähler potentials which are used in string compactifications on orbifold spaces.

Acknowledgements: We are grateful to R. Blumenhagen, G. Curio, B. Florea, W. Lerche, P. Mayr, E. Scheidegger and P. Tripathy for useful discussions. This work is supported in part by the Deutsche Forschungsgemeinschaft as well as by the EU-RTN network Constituents, Fundamental Forces and Symmetries of the Universe (MRTN-CT2004-005104). S.R. and W.S. thank the university of Munich for hospitality.

\section{Appendix A. Complex structures of $\mathbf{Z}_{N^{-}}$and $\mathbf{Z}_{N} \times \mathbf{Z}_{M^{-}}$orbifolds}

In this appendix, we will derive the complex structures and Kähler moduli of all the $\mathbf{Z}_{N^{-}}$and $\mathbf{Z}_{N} \times \mathbf{Z}_{M}$-orbifolds listed in tables 1 and 2 which were not treated already in the main text, i.e. $\mathbf{Z}_{6-I}, \mathbf{Z}_{6-I I}, \mathbf{Z}_{7}, \mathbf{Z}_{12-I}, \mathbf{Z}_{3} \times \mathbf{Z}_{3}, \mathbf{Z}_{2} \times \mathbf{Z}_{6}, \mathbf{Z}_{2} \times \mathbf{Z}_{6^{\prime}}$ and $\mathbf{Z}_{6} \times \mathbf{Z}_{6}$.

Throughout the whole section, we shall work with the heterotic anti-symmetric 2form $B_{2}$. We have already explained in section 2, that the 2-form components entering the Kähler moduli have to be replaced by the corresponding part of the Ramond 4-form in the type $I I B$ orientifolds we are discussing. This map is obvious due to Poincaré duality on $X_{6}$. 


\section{A.1. $\mathbf{Z}_{6-I^{-} \text {orbifold }}$}

The action of the $\mathbf{Z}_{6-I^{-}}$twist on the complex coordinates is as follows:

$$
z^{i} \longrightarrow e^{2 \pi i v^{i}} z^{i} \quad, \quad v^{1}=\frac{1}{6}, v^{2}=\frac{1}{6}, v^{3}=-\frac{2}{6} .
$$

$G_{2}^{2} \times S U(3)-$ lattice

On the root lattice of $G_{2}^{2} \times S U(3)$, the twist $Q$ has the following action (see discussion in section 3$)$ :

$$
\begin{aligned}
& Q e_{1}=2 e_{1}+3 e_{2} \quad, \quad Q e_{2}=-e_{1}-e_{2}, \\
& Q e_{3}=2 e_{3}+3 e_{4} \quad, \quad Q e_{4}=-e_{3}-e_{4}, \\
& Q e_{5}=e_{6} \quad, \quad Q e_{6}=-e_{5}-e_{6} .
\end{aligned}
$$

The twist $Q$ allows for five independent real deformations of the metric $g$ and five real deformations of the anti-symmetric tensor $b$. As before, these results follow again from solving the equations $Q^{t} g Q=g$ and $Q^{t} b Q=b$ :

$g=\left(\begin{array}{cccccc}R_{1}^{2} & -\frac{1}{2} R_{1}^{2} & R_{1} R_{3} \cos \theta_{13} & y & 0 & 0 \\ -\frac{1}{2} R_{1}^{2} & \frac{1}{3} R_{1}^{2} & \frac{1}{\sqrt{3}} R_{1} R_{3} \cos \theta_{23} & \frac{1}{3} R_{1} R_{3} \cos \theta_{13} & 0 & 0 \\ R_{1} R_{3} \cos \theta_{13} & \frac{1}{\sqrt{3}} R_{1} R_{3} \cos \theta_{23} & R_{3}^{2} & -\frac{1}{2} R_{3}^{2} & 0 & 0 \\ y & \frac{1}{3} R_{1} R_{3} \cos \theta_{13} & -\frac{1}{2} R_{3}^{2} & \frac{1}{3} R_{3}^{2} & 0 & 0 \\ 0 & 0 & 0 & 0 & R_{5}^{2} & -\frac{1}{2} R_{5}^{2} \\ 0 & 0 & 0 & 0 & -\frac{1}{2} R_{5}^{2} & R_{5}^{2}\end{array}\right)$,

with $y=-\frac{1}{3}\left(3 R_{1} R_{3} \cos \theta_{13}-\sqrt{3} R_{1} R_{3} \cos \theta_{23}\right)$ and the five real parameters $R_{1}^{2}, R_{3}^{2}, R_{5}^{2}, \theta_{13}, \theta_{23}$. For the choice $\theta_{13}=\theta_{23}=\pi / 2$, we get the metric one obtains from the Cartan matrices back. For $b$ we find

$$
b=\left(\begin{array}{cccccc}
0 & b_{1} & 3 b_{2} & -3 b_{2}-b_{3} & 0 & 0 \\
-b_{1} & 0 & b_{3} & b_{2} & 0 & 0 \\
-3 b_{2} & -b_{3} & 0 & b_{4} & 0 & 0 \\
3 b_{2}+b_{3} & -b_{2} & -b_{4} & 0 & 0 & 0 \\
0 & 0 & 0 & 0 & 0 & b_{5} \\
0 & 0 & 0 & 0 & -b_{5} & 0
\end{array}\right)
$$

with the five real parameters $b_{1}, b_{2}, b_{3}, b_{4}, b_{5}$. We see that we get 5 untwisted Kähler moduli in this orbifold, while the complex structure is completely fixed.

It is possible to fix the complex structure up to a few constants by choosing a suitable ansatz such that it repects the twist without the use of the lattice vectors:

$$
\begin{aligned}
& d z^{1}=a\left(-\left(1+e^{2 \pi i / 6}\right) d x^{1}+d x^{2}\right)+b\left(-\left(1+e^{2 \pi i / 6}\right) d x^{3}+d x^{4}\right), \\
& d z^{2}=c\left(-\left(1+e^{2 \pi i / 6}\right) d x^{1}+d x^{2}\right)+d\left(-\left(1+e^{2 \pi i / 6}\right) d x^{3}+d x^{4}\right), \\
& d z^{3}=e\left(e^{-2 \pi i / 3} d x^{5}+d x^{6}\right) .
\end{aligned}
$$


$a, b, c, d$ and $e$ are constants left unfixed by the twist alone. In the following, we will choose $a, d$, e such that $d x^{1}, d x^{3}, d x^{5}$ have the coefficient one and set $b=c=0$, so the complex structure takes the following form:

$$
\begin{aligned}
& d z^{1}=d x^{1}+\frac{1}{\sqrt{3}} e^{5 \pi i / 6} d x^{2}, \\
& d z^{2}=d x^{3}+\frac{1}{\sqrt{3}} e^{5 \pi i / 6} d x^{4}, \\
& d z^{3}=3^{1 / 4}\left(d x^{5}+e^{2 \pi i / 3} d x^{6}\right) .
\end{aligned}
$$

Now we proceed as outlined in section 2. From the metric (A.3) we can easily read off the Kähler form, which expressed in the complex coordinates (A.6) reads

$$
\begin{aligned}
-i J= & R_{1}^{2} d z^{1} \wedge d \bar{z}^{1}+R_{3}^{2} d z^{2} \wedge d \bar{z}^{2}+\frac{1}{\sqrt{3}} R_{5}^{2} d z^{3} \wedge d \bar{z}^{3} \\
& +2 R_{1} R_{3}\left[\left(e^{2 \pi i / 6} \cos \theta_{13}+i \cos \theta_{23}\right) d z^{2} \wedge d \bar{z}^{1}+\left(e^{-2 \pi i / 6} \cos \theta_{13}-i \cos \theta_{23}\right) d z^{1} \wedge d \bar{z}^{2}\right] .
\end{aligned}
$$

To be able to read off the Kähler moduli, we must look at the real cohomology. The five untwisted $(1,1)$-forms that are invariant under this orbifold twist are

$$
\begin{aligned}
& \omega_{1}=d x^{1} \wedge d x^{2}, \quad \omega_{2}=d x^{3} \wedge d x^{4}, \quad \omega_{3}=d x^{5} \wedge d x^{6} \\
& \omega_{4}=d x^{2} \wedge d x^{3}-d x^{1} \wedge d x^{4}, \quad \omega_{5}=3 d x^{1} \wedge d x^{3}-3 d x^{1} \wedge d x^{4}+d x^{2} \wedge d x^{4} .
\end{aligned}
$$

The $B$-field (A.4) has the simple form

$$
B=b_{1} \omega_{1}+b_{4} \omega_{2}+b_{5} \omega_{3}+b_{3} \omega_{4}+b_{2} \omega_{5}
$$

The Kähler form expanded in the real cohomology is

$$
\begin{aligned}
J= & \frac{1}{2 \sqrt{3}}\left\{R_{1}^{2} \omega_{1}+R_{3}^{2} \omega_{2}+3 R_{5}^{2} \omega_{3}\right. \\
& \left.-2 R_{1} R_{3}\left[\cos \theta_{13} \omega_{4}-\frac{2}{\sqrt{3}}\left(13 \sqrt{3} \cos \theta_{13}+29 \cos \theta_{23}\right) \omega_{5}\right]\right\}
\end{aligned}
$$

Via $J+i B=\mathcal{T}^{i} \omega_{i}$ the Kähler moduli can now be easily read off:

$$
\begin{aligned}
& \mathcal{T}^{1}=\frac{1}{2 \sqrt{3}} R_{1}^{2}+i b_{1}, \quad \mathcal{T}^{2}=\frac{1}{2 \sqrt{3}} R_{3}^{2}+i b_{4}, \quad \mathcal{T}^{3}=\frac{\sqrt{3}}{2} R_{5}^{2}+i b_{5} \\
& \mathcal{T}^{4}=-\frac{1}{\sqrt{3}} R_{1} R_{3} \cos \theta_{13}+i b_{3}, \quad \mathcal{T}^{5}=\frac{1}{3} R_{1} R_{3}\left(13 \sqrt{3} \cos \theta_{13}+29 \cos \theta_{23}\right)+b_{2}
\end{aligned}
$$

$G_{2} \times(S U(3))^{2}$-lattice with generalized Coxeter element 
On the root lattice of $G_{2} \times(S U(3))^{2}$, we act with the generalized Coxeter twist $Q=S_{1} S_{2} S_{3} S_{4} P_{36} P_{45}$, as explained in section 3 . It has the following action:

$$
\begin{aligned}
& Q e_{1}=2 e_{1}+3 e_{2} \quad, \quad Q e_{2}=-e_{1}-e_{2}, \\
& Q e_{3}=e_{6} \quad, \quad Q e_{4}=e_{5}, \\
& Q e_{5}=-e_{3}-e_{4} \quad, \quad Q e_{6}=e_{4} .
\end{aligned}
$$

As before, the twist $Q$ allows for five independent real deformations of the metric $g$ and five real deformations of the anti-symmetric tensor $b$ :

$$
g=\left(\begin{array}{cccccc}
R_{1}^{2} & -\frac{1}{2} R_{1}^{2} & x & -x & -x & x \\
-\frac{1}{2} R_{1}^{2} & \frac{1}{3} R_{1}^{2} & -y & x & y & z \\
x & -y & R_{5}^{2} & -\frac{1}{2} R_{5}^{2} & -2 R_{5}^{2} \cos \theta_{46} & R_{5}^{2} \cos \theta_{46} \\
-x & x & -\frac{1}{2} R_{5}^{2} & R_{5}^{2} & R_{5}^{2} \cos \theta_{46} & R_{5}^{2} \cos \theta_{46} \\
-x & y & -2 R_{5}^{2} \cos \theta_{46} & R_{5}^{2} \cos \theta_{46} & R_{5}^{2} & -\frac{1}{2} R_{5}^{2} \\
x & z & R_{5}^{2} \cos \theta_{46} & R_{5}^{2} \cos \theta_{46} & -\frac{1}{2} R_{5}^{2} & R_{5}^{2}
\end{array}\right),
$$

with $x=\frac{1}{\sqrt{3}}\left(R_{1} R_{5}\left(\cos \theta_{25}+\cos \theta_{26}\right), y=\frac{1}{\sqrt{3}} R_{1} R_{5} \cos \theta_{25}, z=\frac{1}{\sqrt{3}} R_{1} R_{5} \cos \theta_{26}\right.$ and the five real parameters $R_{1}^{2}, R_{5}^{2}, \theta_{25}, \theta_{26}$ and $\theta_{46}$. For $b$ we find

$$
b=\left(\begin{array}{cccccc}
0 & b_{1} & 2 b_{2}+b_{3} & -b_{2}-2 b_{3} & -2 b_{2}-b_{3} & b_{2}-b_{3} \\
-b_{1} & 0 & -b_{2} & b_{2}+b_{3} & b_{2} & b_{3} \\
-2 b_{2}-b_{3} & b_{2} & 0 & -b_{5} & 0 & -b_{4} \\
b_{2}+2 b_{3} & -b_{2}-b_{3} & b_{5} & 0 & -b_{4} & b_{4} \\
2 b_{2}+b_{3} & -b_{2} & 0 & b_{4} & 0 & b_{5} \\
-b_{2}+b_{3} & -b_{2} & b_{4} & -b_{4} & -b_{5} & 0
\end{array}\right)
$$

with the five real parameters $b_{1}, b_{2}, b_{3}, b_{4}, b_{5}$.

For the complex structure, we find

$$
\begin{aligned}
& d z^{1}=a\left(-\left(1+e^{2 \pi i / 6}\right) d x^{1}+d x^{2}\right)+b\left(e^{-2 \pi i / 3} d x^{3}+d x^{4}+e^{2 \pi i / 6} d x^{5}+e^{-2 \pi i / 6} d x^{6}\right), \\
& d z^{2}=c\left(-\left(1+e^{2 \pi i / 6}\right) d x^{1}+d x^{2}\right)+d\left(e^{-2 \pi i / 3} d x^{3}+d x^{4}+e^{2 \pi i / 6} d x^{5}+e^{-2 \pi i / 6} d x^{6}\right), \\
& d z^{3}=e\left(e^{-2 \pi i / 3} d x^{3}+d x^{4}+e^{-2 \pi i / 3} d x^{5}+e^{2 \pi i / 3} d x^{6}\right) .
\end{aligned}
$$

$a, b, c, d$ and $e$ are constants left unfixed by the twist. We choose them such that we get

$$
\begin{aligned}
& d z^{1}=3^{-1 / 3}\left(d x^{1}+\frac{1}{\sqrt{3}} e^{5 \pi i / 6} d x^{2}\right) \\
& d z^{2}=\frac{1}{\sqrt{2}}\left(d x^{3}+e^{2 \pi i / 3} d x^{4}-d x^{5}+e^{2 \pi i / 6} d x^{6}\right) \\
& d z^{3}=\frac{1}{\sqrt{2}}\left(d x^{3}+e^{2 \pi i / 3} d x^{4}+d x^{5}-e^{2 \pi i / 6} d x^{6}\right) .
\end{aligned}
$$


The five untwisted $(1,1)$-forms that are invariant under this orbifold twist are

$$
\begin{aligned}
& \omega_{1}=d x^{1} \wedge d x^{2}, \\
& \omega_{2}=2 d x^{1} \wedge d x^{3}-d x^{1} \wedge d x^{4}-2 d x^{1} \wedge d x^{5}+d x^{1} \wedge d x^{6}-d x^{2} \wedge d x^{3}+d x^{2} \wedge d x^{4}+d x^{2} \wedge d x^{5}, \\
& \omega_{3}=d x^{1} \wedge d x^{3}-2 d x^{1} \wedge d x^{4}-d x^{1} \wedge d x^{5}-d x^{1} \wedge d x^{6}+d x^{2} \wedge d x^{4}+d x^{2} \wedge d x^{6}, \\
& \omega_{4}=d x^{3} \wedge d x^{6}+d x^{4} \wedge d x^{5}-d x^{4} \wedge d x^{6}, \\
& \omega_{5}=d x^{5} \wedge d x^{6} .
\end{aligned}
$$

The $B$-field (A.14) has the simple form $B=b_{1} \omega_{1}+b_{2} \omega_{2}+b_{3} \omega_{3}+b_{4} \omega_{4}+b_{5} \omega_{5}$. Looking at the Kähler form expanded in the real cohomology and with $J+i B=\mathcal{T}^{i} \omega_{i}$ the Kähler moduli are found to be

$$
\begin{aligned}
\mathcal{T}^{1} & =\frac{1}{2 \sqrt{3}} R_{1}^{2}+i b_{1}, \quad \mathcal{T}^{2}=\frac{1}{3} R_{1} R_{5}\left(\cos \theta_{25}+20 \cos \theta_{26}\right)+i b_{2}, \\
\mathcal{T}^{3} & =-R_{1} R_{5}\left(4 \cos \theta_{25}-\cos \theta_{26}\right)+i b_{3}, \\
\mathcal{T}^{4} & =\frac{3 \sqrt{3}}{2} R_{5}^{2} \cos \theta_{46}+i b_{4}, \quad \mathcal{T}^{5}=-\sqrt{3} R_{5}^{2}+i b_{5} .
\end{aligned}
$$

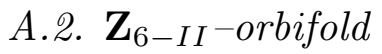

The action of the $\mathbf{Z}_{6-I I^{-}}$twist on the complex coordinates is as follows:

$$
z^{i} \longrightarrow e^{2 \pi i v^{i}} z^{i} \quad, \quad v^{1}=\frac{1}{6}, v^{2}=\frac{2}{6}, v^{3}=-\frac{3}{6}
$$

$$
\mathbf{Z}_{6-I I} \text { on }(S U(2))^{2} \times S U(3) \times G_{2}
$$

The lattices of $G_{2}$ and $S U(3)$ are each two-dimensional, while the lattice of $S U(2)$ is one-dimensional. We associate the lattice basis vectors $e_{1}, e_{2}$ with $G_{2}, e_{3}, e_{4}$ with $\mathrm{SU}(3)$ and $e_{5}, e_{6}$ with the two $S U(2)$ s. The orbifold twist expressed in the complex basis reads

$$
z^{i} \longrightarrow e^{2 \pi i v^{i}} z^{i} \quad, \quad v^{1}=\frac{1}{6}, v^{2}=\frac{2}{6}, v^{3}=-\frac{3}{6}
$$

When examining (A.20), we see that the twist of $z^{3}$ is merely a $\mathbf{Z}_{2}$-reflection. It must be associated with the one-dimensional lattices $S U(2)$. The twist action for this case is $e_{5} \rightarrow-e_{5}, \quad e_{6} \rightarrow-e_{6}$.

The twist on $z^{2}$ corresponds to a $\mathbf{Z}_{3}$-action. This twist must clearly live on the $S U(3)$-lattice, whose simple roots are $(1,0)$ and $(-1 / 2, \sqrt{3} / 2)$. The action of the Coxeter element of $S U(3)$ results in $e_{3} \rightarrow e_{4}, \quad e_{4} \rightarrow-e_{3}-e_{4}$. 
The twist on $z^{1}$ is the $\mathbf{Z}_{6}$ twist, living on the $G_{2}$-root lattice. The Coxeter element acts as $e_{1} \rightarrow 2 e_{1}+3 e_{2}, \quad e_{2} \rightarrow-e_{1}-e_{2}$ which fulfills $q^{6}=1$. So the full twist $Q$ acts on the six roots $e_{i}$ in the following way:

$$
\begin{aligned}
& Q e_{1}=2 e_{1}+3 e_{2}, \quad Q e_{2}=-e_{1}-e_{2}, \\
& Q e_{3}=e_{4}, \quad Q e_{4}=-e_{3}-e_{4}, \\
& Q e_{i}=-e_{i}, \quad i=5,6
\end{aligned}
$$

Now we solve for the metric and antisymmetric tensor, using $Q^{t} g Q=g$ and $Q^{t} b Q=b$. We find the following solution for $g$ (which is incidentally the same we get directly from the Cartan matrix):

$$
g=\left(\begin{array}{cccccc}
R_{1}^{2} & -1 / 2 R_{1}^{2} & 0 & 0 & 0 & 0 \\
-1 / 2 R_{1}^{2} & 1 / 3 R_{1}^{2} & 0 & 0 & 0 & 0 \\
0 & 0 & R_{3}^{2} & -1 / 2 R_{3}^{3} & 0 & 0 \\
0 & 0 & -1 / 2 R_{3}^{2} & R_{3}^{2} & 0 & 0 \\
0 & 0 & 0 & 0 & R_{5}^{2} & R_{5} R_{6} \cos \left(\theta_{56}\right) \\
0 & 0 & 0 & 0 & R_{5} R_{6} \cos \left(\theta_{56}\right) & R_{6}^{2}
\end{array}\right) .
$$

As can be seen, $R_{1}, R_{3}, R_{5}, R_{6}$ and $\theta_{56}$ are (real) free parameters. The $\mathbf{Z}_{2}$-twists leave their part of the metric completely undetermined. For the antisymmetric tensor $b$, we get

$$
b=\left(\begin{array}{cccccc}
0 & b_{1} & 0 & 0 & 0 & \\
-b_{1} & 0 & 0 & 0 & 0 & 0 \\
0 & 0 & 0 & b_{2} & 0 & 0 \\
0 & 0 & -b_{2} & 0 & 0 & 0 \\
0 & 0 & 0 & 0 & 0 & b_{3} \\
0 & 0 & 0 & 0 & -b_{3} & 0
\end{array}\right)
$$

with the three real parameters $b_{1}, b_{2}, b_{3}$. Three of the free parameters of $g$ can be combined with the three free parameters of $b$ into three complex Kähler moduli, the remaining two free parameters of $g$ form one complex structure modulus.

Using the ansatz (2.14) and normalizing the first term to one, we find the following complex structure:

$$
\begin{aligned}
& d z^{1}=d x^{1}+\frac{1}{\sqrt{3}} e^{5 \pi i / 6} d x^{2}, \\
& d z^{2}=d x^{3}+e^{2 \pi i / 3} d x^{4}, \\
& d z^{3}=\frac{1}{\sqrt{2 \operatorname{Im} \mathcal{U}^{3}}}\left(d x^{5}+\mathcal{U}^{3} d x^{6}\right), \quad \text { with } \quad \mathcal{U}^{3}=\frac{R_{6}}{R_{5}} e^{i \theta_{56}}
\end{aligned}
$$

the complex structure modulus. The invariant 2 -forms are in this relatively simple case $d x^{1} \wedge d x^{2}, d x^{3} \wedge d x^{4}$ and $d x^{5} \wedge d x^{6}$. The Kähler form in complex coordinates has the form

$$
-i J=R_{1}^{2} d z^{1} \wedge d \bar{z}^{1}+R_{3}^{2} d z^{1} \wedge d \bar{z}^{1}+2 R_{5} R_{6} \sin \theta_{56} d z^{3} \wedge d \bar{z}^{3} .
$$


Expressed in the real cohomology, it takes the form

$$
J=\frac{1}{2 \sqrt{3}} R_{1}^{2} d x^{1} \wedge d x^{2}+\frac{\sqrt{3}}{2} R_{3}^{2} d x^{3} \wedge d x^{4}+R_{5} R_{6} \sin \theta_{56} d x^{5} \wedge d x^{6},
$$

so via $J+i B=\mathcal{T}^{i} \omega_{i}$, we find the three Kähler moduli to take the following form:

$$
\begin{aligned}
& \mathcal{T}^{1}=\frac{1}{2 \sqrt{3}} R_{1}^{2}+i b_{1}, \\
& \mathcal{T}^{2}=\frac{\sqrt{3}}{2} R_{3}^{2}+i b_{2}, \\
& \mathcal{T}^{3}=R_{5} R_{6} \sin \theta_{56}+i b_{3} .
\end{aligned}
$$

$S U(6) \times S U(2)$-lattice

On the root lattice of $S U(6) \times S U(2)$, the twist $Q$ acts on the six roots $e_{i}$ in the following way:

$$
\begin{aligned}
& Q e_{i}=e_{i+1} \quad, \quad i=1, \ldots 4, \\
& Q e_{5}=-e_{1}-e_{2}-e_{3}-e_{4}-e_{5}, \\
& Q e_{6}=-e_{6} .
\end{aligned}
$$

The twist $Q$ allows for five independent real deformations of the metric $g$ and three real deformations of the anti-symmetric tensor $b$. As before, these results follow from solving the equations $Q^{t} g Q=g$ and $Q^{t} b Q=b$ which leads to:

$$
g=\left(\begin{array}{cccccc}
R_{1}^{2} & R_{1}^{2} x & R_{1}^{2} \alpha_{15} & R_{1}^{2} \alpha_{14} & R_{1}^{2} \alpha_{15} & R_{1} R_{6} \alpha_{16} \\
R_{1}^{2} x & R_{1}^{2} & R_{1}^{2} x & R_{1}^{2} \alpha_{15} & R_{1}^{2} \alpha_{14} & -R_{1} R_{6} \alpha_{16} \\
R_{1}^{2} \alpha_{15} & R_{1}^{2} x & R_{1}^{2} & R_{1}^{2} x & R_{1}^{2} \alpha_{15} & R_{1} R_{6} \alpha_{16} \\
R_{1}^{2} \alpha_{14} & R_{1}^{2} \alpha_{15} & R_{1}^{2} x & R_{1}^{2} & R_{1}^{2} x & -R_{1} R_{6} \alpha_{16} \\
R_{1}^{2} \alpha_{15} & R_{1}^{2} \alpha_{14} & R_{1}^{2} \alpha_{15} & R_{1}^{2} x & R_{1}^{2} & R_{1} R_{6} \alpha_{16} \\
R_{1} R_{6} \alpha_{16} & -R_{1} R_{6} \alpha_{16} & R_{1} R_{6} \alpha_{16} & -R_{1} R_{6} \alpha_{16} & R_{1} R_{6} \alpha_{16} & R_{6}^{2}
\end{array}\right),
$$

$x=-\frac{1}{2}\left(1+\alpha_{14}+2 \alpha_{15}\right)$, with the arbitrary real parameters $R_{1}^{2}, R_{6}^{2}, \alpha_{14}, \alpha_{15}, \alpha_{16}$ and

$$
b=\left(\begin{array}{cccccc}
0 & b_{1} & b_{2} & 0 & -b_{2} & b_{3} \\
-b_{1} & 0 & b_{1} & b_{2} & 0 & -b_{3} \\
-b_{2} & -b_{1} & 0 & b_{1} & b_{2} & b_{3} \\
0 & -b_{2} & -b_{1} & 0 & b_{1} & -b_{3} \\
b_{2} & 0 & -b_{2} & -b_{1} & 0 & b_{3} \\
-b_{3} & b_{3} & -b_{3} & b_{3} & -b_{3} & 0
\end{array}\right)
$$

with the arbitrary real parameters $b_{1}, b_{2}, b_{3}$. The $\sigma$-model action for the closed string reads in this lattice basis:

$$
\mathcal{S}=\int d^{2} z \bar{\partial} x^{i}(\bar{z})(g+b)_{i j} \partial x^{j}(z) .
$$


The aim is to transform the action (A.31) into a complex basis $\left\{z^{i}\right\}_{i=1,2,3}$, where the twist $Q$ acts diagonally on the complex coordinates. There are several such bases, the second requirement is that the three Kähler moduli $\mathcal{T}^{j}$ and complex structure moduli have to decouple from each other.

In the complex basis, the $\sigma$-model has the following action $\mathrm{\theta}$ :

$$
\mathcal{S}=\frac{1}{2} \int d^{2} z\left[b_{(1,1)}{ }_{i \bar{j}} \partial z^{i} \bar{\partial} \bar{z}^{j}+\text { h.c }\right],
$$

where $b_{(1,1)}$ is the matrix containing the Kähler moduli, i.e. $(g+b)$ transformed to the complex basis. The action (A.32) must be invariant under the twist and the form of the matrix $b_{(1,1)}$ changes depending on the twist. Again, we transform the action (A.31) into a complex basis $\left\{z^{i}\right\}_{i=1,2,3}$, where the twist $Q$ acts diagonally on the complex coordinates. We use the vielbein

$$
\begin{aligned}
e_{i} & =\sum_{j=1,3,5} A_{j}\left\{\cos \left[(i-1) \kappa_{j} \alpha+\phi_{j}\right] \widetilde{e}_{j}+\sin \left[(i-1) \kappa_{j} \alpha+\phi_{j}\right] \widetilde{e}_{j+1}\right\} \quad, \quad i=1, \ldots, 5, \\
e_{6} & =R_{6}\left[\cos \left(\Delta+\phi_{5}\right) \widetilde{e}_{5}+\sin \left(\Delta+\phi_{5}\right) \widetilde{e}_{6}\right],
\end{aligned}
$$

with

$$
\begin{aligned}
A_{1}^{2} & =\frac{1}{6} R_{1}^{2}\left(1-3 \alpha_{14}-4 \alpha_{15}\right), \\
A_{3}^{2} & =\frac{1}{2} R_{1}^{2}\left(1+\alpha_{14}\right), \\
A_{5}^{2} & =\frac{1}{3} R_{1}^{2}\left(1+2 \alpha_{15}\right), \\
\cos (\Delta) & =\frac{\sqrt{3} \alpha_{16}}{\sqrt{1+2 \alpha_{15}}},
\end{aligned}
$$

and $\alpha=\frac{\pi}{3}, \kappa_{1}=1, \kappa_{3}=2$ and $\kappa_{5}=3$. The angles $\phi_{i}$ are arbitrary reflecting the freedom of how to embed our six-dimensional lattice into the orthonormal system $\left\{\widetilde{e}_{i}\right\}_{i=1, \ldots, 6}$. After having moved into the real orthonormal basis $\left\{\widetilde{e}_{i}\right\}_{i=1, \ldots, 6}$, we have to find the unitary transformation, which brings us to the complex basis $\left\{z^{i}\right\}_{i=1,2,3}$. Here, we find:

$$
\begin{aligned}
& d z^{1}=d x^{1}+e^{2 \pi i / 6} d x^{2}+e^{2 \pi i / 3} d x^{3}-d x^{4}+e^{-2 \pi i / 3} d x^{5} \\
& d z^{2}=d x^{1}+e^{2 \pi i / 3} d x^{2}+e^{-2 \pi i / 3} d x^{3}+d x^{4}+e^{2 \pi i / 3} d x^{5} \\
& d z^{3}=\frac{1}{2 \sqrt{3}}\left[\frac{1}{3}\left(d x^{1}-d x^{2}+d x^{3}-d x^{4}+d x^{5}\right)+\mathcal{U}^{3} d x^{6}\right] .
\end{aligned}
$$

8 In the case of rectangular complex coordinates, i.e. $z^{i}=x^{i}+i y^{i}$, the action takes the form $\mathcal{S}=\frac{1}{2} \int d^{2} z\left[b_{(1,2)}{ }_{i j} \partial z^{i} \bar{\partial} z^{j}+b_{(1,1)} i \bar{j} \partial z^{i} \bar{\partial} \bar{z}^{\bar{j}}+\right.$ h.c $]$, where $b_{(1,2)}$ is the matrix containing the complex structure moduli. The form of the matrices is determined via the twist, and the number of untwisted moduli is manifest in the action (see e.g. 433). 
We have set the three free angles $\phi_{i}$ to zero after having realized, that they act on each $z^{i}$ just as an overall phase. In the basis (A.35) the $\sigma$-model action (A.32) takes the form

$$
\mathcal{S}=\frac{1}{2} \int d^{2} z \sum_{j=1}^{3} \mathcal{T}^{j} \bar{\partial} \bar{z}^{j} \partial z^{j}+\text { h.c. }
$$

where we defined $\mathcal{T}^{i}=b_{(1,1)_{i} \bar{i}}$ and $\mathcal{U}^{3}=b_{(1,2)}$, with the three Kähler moduli $\mathcal{T}^{i}:$ 国

$$
\begin{aligned}
& \mathcal{T}^{1}=R_{1}^{2}\left(1-3 \alpha_{14}-4 \alpha_{15}\right)+2 i \sqrt{3}\left(b_{1}+b_{2}\right) \\
& \mathcal{T}^{2}=\sqrt{3} R_{1}^{2}\left(1+\alpha_{14}\right)+2 i\left(b_{1}-b_{2}\right) \\
& \mathcal{T}^{3}=\sqrt{3} R_{1} R_{6} \sqrt{1+2 \alpha_{15}-3 \alpha_{16}^{2}}+3 i b_{3}
\end{aligned}
$$

and the complex structure modulus $\mathcal{U}^{3}$ :

$$
\mathcal{U}^{3}=\frac{R_{6}}{R_{1}} \frac{\alpha_{16}+\frac{i}{3} \sqrt{3} \sqrt{1+2 \alpha_{15}-3 \alpha_{16}^{2}}}{1+2 \alpha_{15}}
$$

The $(S U(2))^{2} \times(S U(3))^{2}$-lattice with generalized Coxeter element

This time, we associate $e_{1}$ and $e_{2}$ with $\mathbf{Z}_{2}$. The generalized Coxeter element $Q=$ $S_{1} S_{2} S_{3} P_{36} P_{45}$ contains transpositions of the roots of the $S U(3)$-factors. Using (2.11) and the Cartan matrix of $S U(3)$, we find the following for the total twist:

$$
\begin{aligned}
& Q e_{1}=-e_{1}, \quad Q e_{2}=-e_{2}, \\
& Q e_{3}=-e_{5}, \quad Q e_{4}=-e_{5}+e_{6}, \\
& Q e_{5}=e_{4}, \quad Q e_{6}=e_{3} .
\end{aligned}
$$

9 The three invariant 2 -forms of the real cohomology are in this case

$$
\begin{aligned}
& \omega_{1}=d x^{1} \wedge d x^{2}+d x^{2} \wedge d x^{3}+d x^{3} \wedge d x^{4}+d x^{4} \wedge d x^{5} \\
& \omega_{2}=d x^{1} \wedge d x^{3}-d x^{1} \wedge d x^{5}+d x^{2} \wedge d x^{4}+d x^{3} \wedge d x^{5} \\
& \omega_{3}=d x^{1} \wedge d x^{6}-d x^{2} \wedge d x^{6}+d x^{3} \wedge d x^{6}-d x^{4} \wedge d x^{6}+d x^{5} \wedge d x^{6}
\end{aligned}
$$

It is possible to re-parameterize this result obtained via the vielbein to the language of the other examples presented. 
From $Q^{t} g Q=g$ we find the following $g$ :

$g=\left(\begin{array}{cccccc}R_{1}^{2} & R_{1} R_{2} \cos \theta_{12} & 0 & 0 & 0 & 0 \\ R_{1} R_{2} \cos \theta_{12} & R_{3}^{2} & 0 & 0 & 0 & 0 \\ 0 & 0 & R_{2}^{2} & -\frac{1}{2} R_{3}^{2} & -2 R_{3}^{2} \cos \theta_{46} & R_{3}^{2} \cos \theta_{46} \\ 0 & 0 & -\frac{1}{2} R_{3}^{2} & R_{3}^{2} & R_{3}^{2} \cos \theta_{46} & R_{3}^{2} \cos \theta_{46} \\ 0 & 0 & -2 R_{3}^{2} \cos \theta_{46} & R_{3}^{2} \cos \theta_{46} & R_{3}^{2} & -\frac{1}{2} R_{3}^{2} \\ 0 & 0 & R_{3}^{2} \cos \theta_{46} & R_{3}^{2} \cos \theta_{46} & -\frac{1}{2} R_{3}^{2} & R_{3}^{2}\end{array}\right)$,

$R_{1}, R_{2}, R_{3}, \theta_{12}$ and $\theta_{46}$ being its five real deformation parameters. For the antisymmetric tensor $b$, we get

$$
b=\left(\begin{array}{cccccc}
0 & b_{1} & 0 & 0 & 0 & \\
-b_{1} & 0 & 0 & 0 & 0 & 0 \\
0 & 0 & 0 & -b_{3} & 0 & b_{2} \\
0 & 0 & b_{3} & 0 & b_{2} & -b_{2} \\
0 & 0 & 0 & -b_{2} & 0 & b_{3} \\
0 & 0 & -b_{2} & b_{2} & -b_{3} & 0
\end{array}\right)
$$

with the three real parameters $b_{1}, b_{2}, b_{3}$. This leads to the complex structure

$d z^{1}=\frac{1}{\sqrt{3}}\left(d x^{3}-e^{2 \pi i / 3} d x^{4}\right), \quad d z^{2}=\frac{1}{2}\left(d x^{5}-e^{2 \pi i / 3} d x^{6}\right), \quad d z^{3}=\frac{1}{\sqrt{2 \operatorname{Im} \mathcal{U}^{3}}}\left(d x^{1}+\mathcal{U}^{3} d x^{2}\right)$,

with $\mathcal{U}^{3}=R_{2} / R_{1} e^{i \theta_{12}}$. The invariant real 2-forms are

$$
\begin{aligned}
& \omega_{1}=d x^{1} \wedge d x^{2}, \quad \omega_{2}=d x^{3} \wedge d x^{6}+d x^{4} \wedge d x^{5}-d x^{4} \wedge d x^{6} \\
& \omega_{3}=-d x^{3} \wedge d x^{4}+d x^{5} \wedge d x^{6}
\end{aligned}
$$

Via $B+i J=\mathcal{T}^{i} \omega_{i}$, we find

$$
\mathcal{T}^{1}=b_{1}+R_{1} R_{2} \sin \theta_{12}, \quad \mathcal{T}^{2}=b_{2}+i \frac{3}{\sqrt{3}} 2 R_{3}^{2}, \quad \mathcal{T}^{3}=b_{3}-i 2 \sqrt{3} R_{3}^{2} \cos \theta_{46}
$$

The $S U(3) \times S O(8)$-lattice

On the root lattice of $S U(3) \times S O(8)$, the twist $Q$ acts on the six roots $e_{i}$ in the following way:

$$
\begin{aligned}
& Q e_{1}=e_{2}, \quad Q e_{2}=e_{1}+e_{2}+e_{3}+e_{4}, \\
& Q e_{3}=-e_{1}-e_{2}-e_{3}, \quad Q e_{4}=-e_{1}-e_{2}-e_{4}, \\
& Q e_{5}=e_{6}, \quad Q e_{6}=-e_{5}-e_{6} .
\end{aligned}
$$


The twist $Q$ allows for five independent real deformations of the metric $g$ and three real deformations of the anti-symmetric tensor $b$ :

$g=\left(\begin{array}{cccccc}x & y & R_{1}^{2} \cos \theta_{13} & R_{3}^{2}+R_{1}^{2}\left(-1+\cos \theta_{13}\right) & 0 & 0 \\ y & x & z & z & 0 & 0 \\ R_{1}^{2} \cos \theta_{13} & z & R_{1}^{2} & R_{1} R_{3} \cos \theta_{34} & 0 & 0 \\ R_{3}^{2}+R_{1}^{2}\left(-1+\cos \theta_{13}\right) & z & R_{1} R_{3} \cos \theta_{34} & R_{3}^{2} & 0 & 0 \\ 0 & 0 & 0 & 0 & R_{5}^{2} & -\frac{1}{2} R_{5}^{2} \\ 0 & 0 & 0 & 0 & -\frac{1}{2} R_{5}^{2} & R_{5}^{2}\end{array}\right)$,

with $x=R_{1}^{2} \cos \theta_{13}+R_{3}\left(R_{3}+R_{1} \cos \theta_{34}\right), y=-\frac{1}{2}\left(R_{1}^{2}-2 R_{3}^{2}-R_{1}\left(3 R_{1} \cos \theta_{13}+\right.\right.$ $\left.\left.R_{3} \cos \theta_{34}\right)\right), z=-\frac{1}{2} R_{1}\left(R_{1}+R_{1} \cos \theta_{13}+R_{3} \cos \theta_{34}\right)$ and the arbitrary real parameters $R_{1}^{2}, R_{3}^{2} R_{5}^{2}, \theta_{13}, \theta_{34}$ and

$$
b=\left(\begin{array}{cccccc}
0 & b_{1}+b_{2} & -b_{2} & b_{2} & 0 & 0 \\
-b_{1}-b_{2} & 0 & b_{1}+2 b_{2} & b_{1} & 0 & 0 \\
b_{2} & -b_{1}-2 b_{2} & 0 & b_{2} & 0 & 0 \\
-b_{2} & -b_{1} & -b_{2} & 0 & 0 & 0 \\
0 & 0 & 0 & 0 & 0 & b_{3} \\
0 & 0 & 0 & 0 & -b_{3} & 0
\end{array}\right)
$$

This leads to the complex structure

$$
\begin{aligned}
& d z^{1}=d x^{1}+e^{2 \pi i / 6} d x^{2}-d x^{3}-d x^{4}, \quad d z^{2}=d x^{5}+e^{2 \pi i / 3} d x^{6}, \\
& d z^{3}=\frac{1}{\sqrt{\operatorname{Im} \mathcal{U}^{3}}}\left(d x^{1}-d x^{2}+d x^{3}+\mathcal{U}^{3}\left(d x^{1}-d x^{2}+d x^{4}\right)\right)
\end{aligned}
$$

with

$$
\begin{aligned}
\mathcal{U}^{3} & =-2+\frac{1}{R_{1}\left(2+\cos \theta_{13}-R_{3} \cos \theta_{34}\right)}\left[3 R_{1}\left(1+\cos \theta_{13}\right)-i \sqrt{3}\left(R_{1}\right)^{-1 / 2} \times\right. \\
& \times\left(2 R_{1} R_{3}^{2}-R_{1}^{3}+R_{1} \cos \theta_{13}\left(R_{1}^{2}+R_{3}^{2}-2 R_{2} R_{3} \cos \theta_{34}\right)\right. \\
& \left.\left.-R_{3} \cos \theta_{34}\left(R_{3}^{2}-R_{1}^{2}+R_{1} R_{3} \cos \theta_{34}\right)\right)^{1 / 2}\right] .
\end{aligned}
$$

The invariant 2-forms of the real cohomology are

$$
\begin{aligned}
& \omega_{1}=d x^{1} \wedge d x^{2}+d x^{2} \wedge d x^{3}+d x^{2} \wedge d x^{4} \\
& \omega_{2}=d x^{1} \wedge d x^{2}-d x^{1} \wedge d x^{3}+d x^{1} \wedge d x^{4}+2 d x^{2} \wedge d x^{3}+d x^{3} \wedge d x^{4} \\
& \omega_{3}=d x^{5} \wedge d x^{6}
\end{aligned}
$$


With the invariant 2-forms above, we find the following Kähler moduli:

$$
\begin{aligned}
\mathcal{T}^{1} & \left.=\frac{\sqrt{3}}{2} R_{1}\left(R_{1}\left(1-\cos \theta_{13}\right)+R_{3} \cos \theta_{34}\right)\right) \\
\mathcal{T}^{2} & =\frac{1}{2 \sqrt{3}}\left(-3 R_{1}^{2}\left(-1+\cos \theta_{13}\right)+3 R_{1} R_{3} \cos \theta_{34}+10 \sqrt{R_{1}} \times\right. \\
& \times\left(2 R_{1} R_{3}^{2}-R_{1}^{3}+R_{1} \cos \theta_{13}\left(R_{1}^{2}+R_{3}^{2}-2 R_{2} R_{3} \cos \theta_{34}\right)\right. \\
& \left.-R_{3} \cos \theta_{34}\left(R_{3}^{2}-R_{1}^{2}+R_{1} R_{3} \cos \theta_{34}\right)\right)^{1 / 2}, \\
\mathcal{T}^{3} & =\frac{\sqrt{3}}{2} R_{5}^{2} .
\end{aligned}
$$

\section{A.3. $\mathbf{Z}_{7}$-orbifold}

The action of the twist on the complex coordinates has the form

$$
z^{i} \longrightarrow e^{2 \pi i v^{i}} z^{i} \quad, \quad v^{1}=\frac{1}{7}, v^{2}=\frac{2}{7}, v^{3}=-\frac{3}{7} .
$$

Here, the (only possible) torus lattice for the $\mathbf{Z}_{7}$-orbifold is the root lattice of $S U(7)$, with the twist $Q$ acting on the six roots $e_{i}$ in the following way:

$$
\begin{aligned}
& Q e_{i}=e_{i+1} \quad, \quad i=1, \ldots 5 \\
& Q e_{6}=-e_{1}-e_{2}-e_{3}-e_{4}-e_{5}-e_{6} .
\end{aligned}
$$

The twist $Q$ allows for three independent real deformations of the metric $g$ and three real deformations of the anti-symmetric tensor $b$ :

$$
g=R^{2}\left(\begin{array}{cccccc}
1 & \alpha_{12} & \alpha_{13} & x & x & \alpha_{13} \\
\alpha_{12} & 1 & \alpha_{12} & \alpha_{13} & x & x \\
\alpha_{13} & \alpha_{12} & 1 & \alpha_{12} & \alpha_{13} & x \\
x & \alpha_{13} & \alpha_{12} & 1 & \alpha_{12} & \alpha_{13} \\
x & x & \alpha_{13} & \alpha_{12} & 1 & \alpha_{12} \\
\alpha_{13} & x & x & \alpha_{13}^{2} & \alpha_{12} & 1
\end{array}\right) \quad, \quad x=-\frac{1}{2}-\alpha_{12}-\alpha_{13}
$$

with the three real parameter $R^{2}, \alpha_{12}, \alpha_{13}$ and

$$
b=\left(\begin{array}{cccccc}
0 & b_{1} & b_{2} & b_{3} & -b_{3} & -b_{2} \\
-b_{1} & 0 & b_{1} & b_{2} & b_{3} & -b_{3} \\
-b_{2} & -b_{1} & 0 & b_{1} & b_{2} & b_{3} \\
-b_{3} & -b_{2} & -b_{1} & 0 & b_{1} & b_{2} \\
b_{3} & -b_{3} & -b_{2} & -b_{1} & 0 & b_{1} \\
b_{2} & b_{3} & -b_{3} & -b_{2} & -b_{1} & 0
\end{array}\right)
$$

with the three real parameter $b_{1}, b_{2}, b_{3}$. The $\sigma$-model action for the closed string reads in this lattice basis:

$$
\mathcal{S}=\int d^{2} z \bar{\partial} x^{i}(\bar{z})(g+b)_{i j} \partial x^{j}(z)
$$


The aim is to transform the action (A.57) into a complex basis $\left\{z^{i}\right\}_{i=1,2,3}$, where the twist $Q$ acts diagonally on the complex coordinates:

$$
z^{i} \longrightarrow e^{2 \pi i v^{i}} z^{i} \quad, \quad v^{1}=\frac{1}{7}, v^{2}=\frac{2}{7}, v^{3}=-\frac{3}{7}
$$

There are many such bases, but the second requirement is that the three Kähler moduli $\mathcal{T}^{j}$ and complex structure moduli have to decouple from each other. As a first step, the metric (A.55) may be expressed through the Sechsbein e, i.e. $g=e^{t} e$. This may be obtained from Ref. [60], where the lattice vectors $e_{i}$ are expressed as a linear combination of a set of six real orthonormal basis vectors $\widetilde{e}_{i}$

$$
e_{i}=\sum_{j=1,3,5} R_{j}\left\{\cos \left[(i-1) \kappa_{j} \alpha+\phi_{j}\right] \widetilde{e}_{j}+\sin \left[(i-1) \kappa_{j} \alpha+\phi_{j}\right] \widetilde{e}_{j+1}\right\}
$$

with

$$
\begin{aligned}
& R_{1}^{2}=R^{2}\left[\alpha_{12}\left(\alpha_{5}^{2}-\alpha_{1}^{2}\right)+\alpha_{13}\left(\alpha_{5}^{2}-\alpha_{3}^{2}\right)+\frac{1}{2} \alpha_{5}^{2}\right], \\
& R_{3}^{2}=R^{2}\left[\alpha_{12}\left(\alpha_{1}^{2}-\alpha_{3}^{2}\right)+\alpha_{13}\left(\alpha_{1}^{2}-\alpha_{5}^{2}\right)+\frac{1}{2} \alpha_{1}^{2}\right], \\
& R_{5}^{2}=R^{2}\left[\alpha_{12}\left(\alpha_{3}^{2}-\alpha_{5}^{2}\right)+\alpha_{13}\left(\alpha_{3}^{2}-\alpha_{1}^{2}\right)+\frac{1}{2} \alpha_{3}^{2}\right], \\
& \alpha_{i}^{2}=\frac{4}{7}\left[1-\cos \left(b_{i} \alpha\right)\right] \quad, \quad i=1,3,5,
\end{aligned}
$$

and $\alpha=\frac{2 \pi}{7}, \kappa_{1}=1, \kappa_{3}=2$ and $\kappa_{5}=4$. Furthermore, the angles $\phi_{i}$ are arbitrary reflecting the freedom of how to embedd our six-dimensional lattice into the orthonormal system $\left\{\widetilde{e}_{i}\right\}_{i=1, \ldots, 6}$. After having moved into the real orthonormal basis $\left\{\widetilde{e}_{i}\right\}_{i=1, \ldots, 6}$, we have to find the unitary transformation, which brings us to the complex basis $\left\{z^{i}\right\}_{i=1,2,3}$. After some work, we find:

$$
\begin{aligned}
& d z^{1}=d x^{1}+(-1)^{2 / 7} d x^{2}+(-1)^{4 / 7} d x^{3}+(-1)^{6 / 7} d x^{4}-(-1)^{1 / 7} d x^{5}-(-1)^{3 / 7} d x^{6} \\
& d z^{2}=d x^{1}+(-1)^{4 / 7} d x^{2}-(-1)^{1 / 7} d x^{3}-(-1)^{5 / 7} d x^{4}+(-1)^{2 / 7} d x^{5}+(-1)^{6 / 7} d x^{6} \\
& d z^{3}=d x^{1}-(-1)^{1 / 7} d x^{2}+(-1)^{2 / 7} d x^{3}-(-1)^{3 / 7} d x^{4}+(-1)^{4 / 7} d x^{5}-(-1)^{5 / 7} d x^{6}
\end{aligned}
$$

We have set the three free angles $\phi_{i}$ to zero after having realized that they act on each $z^{i}$ just as an overall phase. Invariance under the $Z_{7}$-twist is fulfilled for a $\sigma$-model action of the form

$$
\mathcal{S}=\frac{1}{2} \int d^{2} z \sum_{j=1}^{3} \mathcal{T}^{j} \bar{\partial} \bar{z}^{j}(\bar{z}) \partial z^{j}(z)+\text { hc. }
$$


with the three Kähler moduli:

$$
\begin{aligned}
& \mathcal{T}^{1}=R_{1}^{2}+\frac{4}{7} i\left[b_{3} \sin \left(\frac{\pi}{7}\right)+b_{1} \sin \left(\frac{2 \pi}{7}\right)+b_{2} \sin \left(\frac{3 \pi}{7}\right)\right], \\
& \mathcal{T}^{2}=R_{3}^{2}-\frac{4}{7} i\left[b_{2} \sin \left(\frac{\pi}{7}\right)+b_{3} \sin \left(\frac{2 \pi}{7}\right)-b_{1} \sin \left(\frac{3 \pi}{7}\right)\right], \\
& \mathcal{T}^{3}=R_{5}^{2}-\frac{4}{7} i\left[b_{1} \sin \left(\frac{\pi}{7}\right)-b_{2} \sin \left(\frac{2 \pi}{7}\right)+b_{3} \sin \left(\frac{3 \pi}{7}\right)\right] .
\end{aligned}
$$

From the expression (A.61) we may read off the complex numbers $\tau_{i}^{j}$ and $\rho_{i}^{j}$ appearing in (2.72) (with the replacement $d x^{2 i-1} \rightarrow d x^{i}$ and $d x^{2 i} \rightarrow d y^{i}$ ). In particular, we see that all complex structures are already fixed through the orbifold twist. Hence, no further restrictions follow from flux quantization rules. Furthermore, the Kähler form is given by:

$$
J=\mathcal{T}^{1} d z^{1} \wedge d \bar{z}^{1}+\mathcal{T}^{2} d z^{2} \wedge d \bar{z}^{2}+\mathcal{T}^{3} d z^{3} \wedge d \bar{z}^{3}
$$

Hence, the two flux components, which survive the orbifold twist (cf. Table 4), fulfill the primitivity condition $G_{3} \wedge J=0$.

\section{A.4. $\mathbf{Z}_{12-I^{-}}$orbifold}

The action of the twist on the complex coordinates has the form

$$
z^{i} \longrightarrow e^{2 \pi i v^{i}} z^{i} \quad, \quad v^{1}=\frac{1}{12}, v^{2}=\frac{4}{12}, v^{3}=-\frac{5}{12} .
$$

$S U(3) \times F_{4}$-lattice

Here, the torus lattice is the root lattice of $S U(3) \times F_{4}$. The action of the twist upon the roots is

$$
\begin{aligned}
& Q e_{1}=e_{2}, \quad Q e_{2}=-e_{1}-e_{2}, \quad Q e_{3}=e_{4}, \\
& Q e_{4}=e_{3}+3_{4}+2 e_{5}, \quad Q e_{5}=e_{6}, \quad Q e_{6}=-e_{3}-e_{4}-e_{5}-e_{6} .
\end{aligned}
$$

10 For completeness, we also give the invariant 2-forms of the real cohomology:

$$
\begin{aligned}
& \omega_{1}=d x^{1} \wedge d x^{2}+d x^{2} \wedge d x^{3}+d x^{3} \wedge d x^{4}+d x^{4} \wedge d x^{5}+d x^{5} \wedge d x^{6} \\
& \omega_{2}=d x^{1} \wedge d x^{3}-d x^{1} \wedge d x^{6}+d x^{2} \wedge d x^{4}+d x^{3} \wedge d x^{5}+d x^{4} \wedge d x^{6} \\
& \omega_{3}=d x^{1} \wedge d x^{4}-d x^{1} \wedge d x^{5}+d x^{2} \wedge d x^{5}-d x^{2} \wedge d x^{6}+d x^{3} \wedge d x^{6}
\end{aligned}
$$


The twist allows for 3 real deformations of the metric, which has the form

$$
g=\left(\begin{array}{cccccc}
R_{1}^{2} & -\frac{1}{2} R_{1}^{2} & 0 & 0 & 0 & 0 \\
\frac{1}{2} R_{1}^{2} & R 1^{2} & 0 & 0 & 0 & 0 \\
0 & 0 & R_{3}^{2} & R_{3}^{2} \cos \theta_{34} & x & x \\
0 & 0 & R_{3}^{2} \cos \theta_{34} & R_{3}^{2} & -\frac{1}{2} R_{3}^{2} & x \\
0 & 0 & x & -\frac{1}{2} R_{3}^{2} & \frac{1}{2} R_{3}^{2} & \frac{1}{2} R_{3}^{2} \cos \theta_{34} \\
0 & 0 & x & x & \frac{1}{2} R_{3}^{2} \cos \theta_{34} & \frac{1}{2} R_{3}^{2}
\end{array}\right),
$$

with $x=R_{3}^{2} \cos \theta_{34}$ and $R_{1}, R_{3}$ and $\cos \theta_{34}$ arbitrary free parameters. Also for the $B$-field, the twist allows 3 real deformations:

$$
b=\left(\begin{array}{cccccc}
0 & b_{1} & 0 & 0 & 0 & 0 \\
-b_{1} & 0 & 0 & 0 & 0 & 0 \\
0 & 0 & 0 & 2 b_{3} & b_{2} & -b_{2} \\
0 & 0 & -2 b_{3} & 0 & 2 b_{3} & b_{2} \\
0 & 0 & -b_{2} & -2 b_{3} & 0 & b_{3} \\
0 & 0 & b_{2} & -b_{2} & -b_{3} & 0
\end{array}\right),
$$

with $b_{1}, b_{2}, b_{3}$ the arbitrary parameters, so we have three untwisted Kähler moduli and no complex structure moduli. The three invariant 2 -forms in the real cohomology are

$$
\begin{aligned}
& \omega_{1}=d x^{1} \wedge d x^{2}, \\
& \omega_{2}=d x^{3} \wedge d x^{5}-d x^{3} \wedge d x^{6}+d x^{4} \wedge d x^{6}, \\
& \omega_{3}=2 d x^{3} \wedge d x^{4}+2 d x^{4} \wedge d x^{5}+d x^{5} \wedge d x^{6} .
\end{aligned}
$$

The complex structure turns out to be as follows:

$$
\begin{aligned}
& d z^{1}=3^{-1 / 4}\left(d x^{3}+e^{2 \pi i / 6} d x^{4}+\frac{1}{\sqrt{2}}\left[e^{11 \pi i / 12} d x^{5}+e^{\pi i / 12} d x^{6}\right]\right), \\
& d z^{2}=3^{-1 / 4}\left(d x^{1}+e^{2 \pi i / 3} d x^{2}\right) \\
& d z^{3}=3^{-1 / 4}\left(d x^{3}-e^{2 \pi i / 12} d x^{4}+\frac{1}{\sqrt{2}}\left[e^{5 \pi i / 12} d x^{5}+e^{-5 \pi i / 12} d x^{6}\right]\right) .
\end{aligned}
$$

For the Kähler moduli, we find

$$
\begin{aligned}
\mathcal{T}^{1} & =\frac{\sqrt{3}}{2} R_{1}^{2}+i b_{1}, \\
\mathcal{T}^{2} & =\frac{3}{4} R_{3}^{2}\left(-1+2 \cos \theta_{34}\right)+i b_{2}, \\
\mathcal{T}^{3} & =\frac{9}{4} R_{3}^{2}+i b_{3} .
\end{aligned}
$$

The $E_{6}$-lattice 
When we choose the $E_{6}$-lattice for the torus lattice, the Coxeter twist acts as

$$
\begin{aligned}
& Q e_{1}=e_{2}, \quad Q e_{2}=e_{3}, \quad Q e_{3}=e_{1}+e_{2}+e_{3}+e_{4}+e_{6}, \\
& Q e_{4}=e_{5}, \quad Q e_{5}=-e_{1}-e_{2}-e_{3}-e_{4}-e_{5}, \quad Q e_{6}=-e_{1}-e_{2}-e_{3}-e_{6} .
\end{aligned}
$$

The twist allows for 3 real deformations of the metric, which has the form

$$
g=\left(\begin{array}{cccccc}
R_{5}^{2} & x & \frac{1}{2}\left(R_{6}^{2}-R_{5}^{2}\right) & R_{5}^{2}-R_{6}^{2} & y & -z \\
x & R_{5}^{2} & x & y & R_{5}^{2}-R_{6}^{2} & -z \\
\frac{1}{2}\left(R_{6}^{2}-R_{5}^{2}\right) & x & R_{5}^{2} & -\frac{1}{2} R_{5}^{2} & y & -\frac{1}{2} R_{6}^{2} \\
R_{5}^{2}-R_{6}^{2} & y & -\frac{1}{2} R_{5}^{2} & R_{5}^{2} & x & z \\
y & R_{5}^{2}-R_{6}^{2} & y & x & R_{5}^{2} & z \\
-R_{5} R_{6} \cos \theta_{56} & -z & -\frac{1}{2} R_{6}^{2} & z & z & R_{6}^{2}
\end{array}\right),
$$

with $x=-\frac{1}{2} R_{5}^{2}+R_{5} R_{6} \cos \theta_{56}, y=\frac{1}{2}\left(R_{6}^{2}-R_{5}^{2}\right)-R_{5} R_{6} \cos \theta_{56}, z=R_{5} R_{6} \cos \theta_{56}$ and $R_{5}, R_{6}$ and $\cos \theta_{56}$ arbitrary free parameters. Also for the $B$-field, the twist allows 3 real deformations:

$$
b=\left(\begin{array}{cccccc}
0 & b_{1} & b_{2}-b_{1}-b_{3} & 0 & b_{1}-b_{2} & b_{3} \\
-b_{1} & 0 & b_{1} & b_{2}-b_{1} & 0 & -b_{3} \\
-b_{2}+b_{1}+b_{3} & -b_{1} & 0 & b_{1}-b_{3} & b_{2}-b_{1} & b_{2} \\
0 & -b_{2}+b_{1} & -b_{1}+b_{3} & 0 & b_{1} & -b_{3} \\
b_{2}-b_{1} & 0 & -b_{2}+b_{1} & -b_{1} & 0 & b_{3} \\
-b_{3} & b_{3} & -b_{2} & b_{3} & -b_{3} & 0
\end{array}\right),
$$

with $b_{1}, b_{2}, b_{3}$ the arbitrary parameters, so we have as expected three untwisted Kähler moduli and no complex structure moduli. The invariant 2 -form of the real cohomology are

$$
\begin{aligned}
\omega_{1}= & d x^{1} \wedge d x^{2}-d x^{1} \wedge d x^{3}+d x^{1} \wedge d x^{5}+d x^{2} \wedge d x^{3}-d x^{2} \wedge d x^{4}+d x^{3} \wedge d x^{4} \\
& -d x^{3} \wedge d x^{5}+d x^{4} \wedge d x^{5} \\
\omega_{2}= & d x^{1} \wedge d x^{3}-d x^{1} \wedge d x^{5}+d x^{2} \wedge d x^{4}+d x^{3} \wedge d x^{5}+d x^{3} \wedge d x^{6} \\
\omega_{3}= & -d x^{1} \wedge d x^{3}+d x^{1} \wedge d x^{6}-d x^{2} \wedge d x^{6}-d x^{3} \wedge d x^{4}-d x^{4} \wedge d x^{6}+d x^{5} \wedge d x^{6} .
\end{aligned}
$$

The complex structure is

$$
\begin{aligned}
& d z^{1}=\frac{1}{\sqrt{6}}\left(d x^{1}+e^{2 \pi i / 12} d x^{2}+e^{2 \pi i / 6} d x^{3}-d x^{4}+e^{-10 \pi i / 12} d x^{5}-(1-i) e^{2 \pi i / 6} d x^{6}\right) \\
& d z^{2}=\frac{1}{2}\left(d x^{1}+e^{2 \pi i / 3} d x^{2}+e^{-2 \pi i / 3} d x^{3}+d x^{4}+e^{2 \pi i / 3} d x^{5}\right), \\
& d z^{3}=\frac{1}{3^{1 / 4} \sqrt{6}}\left(d x^{1}+e^{-2 \pi i / 12} d x^{2}+e^{2 \pi i / 6} d x^{3}-d x^{4}+e^{2 \pi i / 12} d x^{5}-(1+i) e^{2 \pi i / 6} d x^{6}\right) .
\end{aligned}
$$


Again we pair $J+i B=\mathcal{T}^{i} \omega_{i}$ in the real cohomology and get the following Kähler moduli:

$$
\begin{aligned}
& \mathcal{T}^{1}=2 \sqrt{3}\left(2 R_{5}^{2}-R_{6}^{2}\right)+i b_{1}, \\
& \mathcal{T}^{2}=-\left(\sqrt{3} R_{5}^{2}+(1+\sqrt{3}) R_{6}^{2}+R_{5} R_{6} \cos \theta_{56}\right)+i b_{2}, \\
& \mathcal{T}^{3}=\frac{5}{6} R_{6}^{2}-6 R_{5} R_{6} \cos \theta_{56}+i b_{3} .
\end{aligned}
$$

\section{A.5. $\mathbf{Z}_{3} \times \mathbf{Z}_{3}$-orbifold}

The two twists act on the complex coordinate as follows:

$$
\begin{aligned}
& Q_{1}: z^{i} \longrightarrow e^{2 \pi i v_{1}^{i}} z^{i} \quad, \quad v_{1}^{1}=\frac{1}{3}, v_{1}^{2}=0, v_{1}^{3}=-\frac{1}{3}, \\
& Q_{2}: z^{i} \longrightarrow e^{2 \pi i v_{2}^{i}} z^{i} \quad, \quad v_{2}^{1}=0, v_{2}^{2}=\frac{1}{3}, v_{2}^{3}=-\frac{1}{3} .
\end{aligned}
$$

The combined twist $Q_{3}=Q_{2} Q_{1}$ is again the well-known $Z_{3}$-twist:

$$
Q_{3}: z^{i} \longrightarrow e^{2 \pi i v_{3}^{i}} z^{i} \quad, \quad v_{3}^{1}=\frac{1}{3}, v_{3}^{2}=\frac{1}{3}, v^{3}=-\frac{2}{3} .
$$

We expect the root lattice of $S U(3) \times S U(3) \times S U(3)$ to be the correct one. Lead by past experience, we choose for the twists acting on the lattice basis:

$$
\begin{aligned}
& Q_{1} e_{1}=e_{2}, \quad Q_{1} e_{2}=-e_{1}-e_{2}, \quad Q_{1} e_{3}=e_{3}, \quad Q_{1} e_{4}=e_{4}, \\
& Q_{1} e_{5}=e_{6}, \quad Q_{1} e_{6}=-e_{5}-e_{6}, \\
& Q_{2} e_{1}=e_{1}, \quad Q_{2} e_{2}=e_{2}, \quad Q_{2} e_{3}=e_{4}, \quad Q_{2} e_{4}=-e_{3}-e_{4}, \\
& Q_{2} e_{5}=e_{6}, \quad Q_{2} e_{6}=-e_{5}-e_{6} .
\end{aligned}
$$

The twists are the usual Coxeter-twists on $S U(3)$ and reproduce the correct eigenvalues and the condition $Q^{3}=1$. The combined twist $Q_{3}$ has the form

$$
\begin{aligned}
& Q_{3} e_{1}=e_{2}, \quad Q_{3} e_{2}=-e_{1}-e_{2}, \\
& Q_{3} e_{3}=e_{4}, \quad Q_{3} e_{4}=-e_{3}-e_{4}, \\
& Q_{3} e_{5}=-e_{5}-e_{6}, \quad Q_{3} e_{6}=e_{5},
\end{aligned}
$$

and also reproduces the required eigenvalues. The twist on $e_{5}, e_{6}$ is the anti-twist of the usual Coxeter-twist. We require the metric to be invariant under all three twists, i.e. we impose the three conditions $Q_{i}^{T} g Q_{i}=g, \quad i=1,2,3$. This leads to the following solution:

$$
g=\left(\begin{array}{cccccc}
R_{1}^{2} & -\frac{1}{2} R_{1}^{2} & 0 & 0 & 0 & 0 \\
-\frac{1}{2} R_{1}^{2} & R_{1}^{2} & 0 & 0 & 0 & 0 \\
0 & 0 & R_{3}^{2} & -\frac{1}{2} R_{3}^{2} & 0 & 0 \\
0 & 0 & -\frac{1}{2} R_{3}^{2} & R_{3}^{2} & 0 & 0 \\
0 & 0 & 0 & 0 & R_{5}^{2} & -\frac{1}{2} R_{5}^{2} \\
0 & 0 & 0 & 0 & -\frac{1}{2} R_{5}^{2} & R_{5}^{2} .
\end{array}\right)
$$


This corresponds exactly to the metric of $S U(3)^{3}$ without any extra degrees of freedom. The solution for $b$ matches the pattern:

$$
b=\left(\begin{array}{cccccc}
0 & b_{1} & 0 & 0 & 0 & 0 \\
-b_{1} & 0 & 0 & 0 & 0 & 0 \\
0 & 0 & 0 & b_{2} & 0 & 0 \\
0 & 0 & -b_{2} & 0 & 0 & 0 \\
0 & 0 & 0 & 0 & 0 & -b_{3} \\
0 & 0 & 0 & 0 & -b_{3} & 0
\end{array}\right)
$$

we therefore know to have three Kähler moduli whereas the complex structure is completely fixed (recall that in the simple $Z_{3}$-twist, we had nine Kähler moduli). For the complex structure we get

$$
\begin{aligned}
& d z^{1}=3^{1 / 4}\left(d x^{1}+e^{2 \pi i / 3} d x^{2}\right), \\
& d z^{2}=3^{1 / 4}\left(d x^{3}+e^{2 \pi i / 3} d x^{4}\right), \\
& d z^{3}=3^{1 / 4}\left(d x^{5}+e^{-2 \pi i / 3} d x^{6}\right) .
\end{aligned}
$$

Examination of the Kähler form yields

$$
\begin{aligned}
\mathcal{T}^{1} & =\frac{\sqrt{3}}{2} R_{1}^{2}+i b_{1}, \\
\mathcal{T}^{2} & =\frac{\sqrt{3}}{2} R_{3}^{2}+i b_{2}, \\
\mathcal{T}^{3} & =\frac{\sqrt{3}}{2} R_{5}^{2}+i b_{3} .
\end{aligned}
$$

\section{A.6. $\mathbf{Z}_{2} \times \mathbf{Z}_{6}$-orbifold}

The two twists act on the complex coordinate as follows:

$$
\begin{aligned}
& Q_{1}: z^{i} \longrightarrow e^{2 \pi i v_{1}^{i}} z^{i} \quad, \quad v_{1}^{1}=\frac{1}{2}, v_{1}^{2}=0, v_{1}^{3}=-\frac{1}{2}, \\
& Q_{2}: z^{i} \longrightarrow e^{2 \pi i v_{2}^{i}} z^{i} \quad, \quad v_{2}^{1}=0, v_{2}^{2}=\frac{1}{6}, v_{2}^{3}=-\frac{2}{6} .
\end{aligned}
$$

The combined twist $Q_{3}=Q_{2} Q_{1}$ is a $Z_{6-I I^{-}}$twist:

$$
Q_{3}: z^{i} \longrightarrow e^{2 \pi i v_{3}^{i}} z^{i} \quad, \quad v_{3}^{1}=-\frac{3}{6}, v_{3}^{2}=\frac{1}{6}, v^{3}=\frac{2}{6} .
$$

We find the root lattice of $S U(2)^{2} \times S U(3) \times G_{2}$ to be the correct one. We choose for the twists acting on the lattice basis:

$$
\begin{aligned}
& Q_{1} e_{1}=-e_{1}, \quad Q_{1} e_{2}=-e_{2}, \quad Q_{1} e_{3}=e_{3}, \quad Q_{1} e_{4}=e_{4}, \\
& Q_{1} e_{5}=-e_{5}, \quad Q_{1} e_{6}=-e_{6}, \\
& Q_{2} e_{1}=e_{1}, \quad Q_{2} e_{2}=e_{2}, \quad Q_{2} e_{3}=2 e_{3}+3 e_{4}, \quad Q_{2} e_{4}=-e_{3}-e_{4}, \\
& Q_{2} e_{5}=-e_{6}, \quad Q_{2} e_{6}=e_{5}+e_{6} .
\end{aligned}
$$


The twists reproduce the correct eigenvalues and the conditions $Q_{1}^{2}=1, Q_{2}^{6}=1$. While the other twists are the usual Coxeter-twists, the $Q_{2}$-twist on $e_{5}, e_{6}$ is a generalized Coxetertwist on $S U(3)$, namely $S_{1} P_{12}$. The combined twist $Q_{3}$ has the form

$$
\begin{aligned}
& Q_{3} e_{1}=-e_{1}, \quad Q_{3} e_{2}=-e_{2}, \\
& Q_{3} e_{3}=2 e_{3}+3 e_{4}, \quad Q_{3} e_{4}=-e_{3}-e_{4}, \\
& Q_{3} e_{5}=e_{6}, \quad Q_{3} e_{6}=-e_{5}-e_{6},
\end{aligned}
$$

and also reproduces the required eigenvalues. We require the metric to be invariant under all three twists, i.e. we impose the three conditions $Q_{i}^{T} g Q_{i}=g, \quad i=1,2,3$. This leads to the following solution:

$$
g=\left(\begin{array}{cccccc}
R_{1}^{2} & R_{1} R_{2} \cos \theta_{12} & 0 & 0 & 0 & 0 \\
R_{1} R_{2} \cos \theta_{12} & R_{1}^{2} & 0 & 0 & 0 & 0 \\
0 & 0 & R_{3}^{2} & -\frac{1}{2} R_{3}^{2} & 0 & 0 \\
0 & 0 & -\frac{1}{2} R_{3}^{2} & \frac{1}{3} R_{3}^{2} & 0 & 0 \\
0 & 0 & 0 & 0 & R_{5}^{2} & -\frac{1}{2} R_{5}^{2} \\
0 & 0 & 0 & 0 & -\frac{1}{2} R_{5}^{2} & R_{5}^{2} .
\end{array}\right)
$$

The solution for $b$ matches the pattern:

$$
b=\left(\begin{array}{cccccc}
0 & b_{1} & 0 & 0 & 0 & 0 \\
-b_{1} & 0 & 0 & 0 & 0 & 0 \\
0 & 0 & 0 & b_{2} & 0 & 0 \\
0 & 0 & -b_{2} & 0 & 0 & 0 \\
0 & 0 & 0 & 0 & 0 & -b_{3} \\
0 & 0 & 0 & 0 & -b_{3} & 0
\end{array}\right),
$$

we therefore know to have three Kähler moduli whereas the complex structure is completely fixed. For the complex structure we get

$$
\begin{aligned}
& d z^{1}=\frac{1}{\sqrt{2 \operatorname{Im} \mathcal{U}^{3}}}\left(d x^{1}+\mathcal{U}^{3} d x^{2}\right), \\
& d z^{2}=d x^{3}+\frac{1}{\sqrt{3}} e^{10 \pi i / 12} d x^{4}, \\
& d z^{3}=d x^{5}+e^{2 \pi i / 3} d x^{6},
\end{aligned}
$$

with $\mathcal{U}^{3}=R_{2} / R_{1} e^{i \theta_{12}}$. Examination of the Kähler form yields

$$
\begin{aligned}
\mathcal{T}^{1} & =R_{1} R_{2} \sin \theta_{12}+i b_{1}, \\
\mathcal{T}^{2} & =\frac{1}{2 \sqrt{3}} R_{3}^{2}+i b_{2}, \\
\mathcal{T}^{3} & =\frac{\sqrt{3}}{2} R_{5}^{2}+i b_{3} .
\end{aligned}
$$




\section{A.7. $\mathbf{Z}_{2} \times \mathbf{Z}_{6^{\prime}-\text { orbifold }}$}

The two twists act on the complex coordinate as follows:

$$
\begin{aligned}
& Q_{1}: z^{i} \longrightarrow e^{2 \pi i v_{1}^{i}} z^{i} \quad, \quad v_{1}^{1}=\frac{1}{2}, v_{1}^{2}=0, v_{1}^{3}=-\frac{1}{2}, \\
& Q_{2}: z^{i} \longrightarrow e^{2 \pi i v_{2}^{i}} z^{i} \quad, \quad v_{2}^{1}=\frac{1}{6}, v_{2}^{2}=\frac{1}{6}, v_{2}^{3}=-\frac{2}{6} .
\end{aligned}
$$

The combined twist $Q_{3}=Q_{2} Q_{1}$ is a $Z_{6-I}$-twist:

$$
Q_{3}: z^{i} \longrightarrow e^{2 \pi i v_{3}^{i}} z^{i} \quad, \quad v_{3}^{1}=-\frac{2}{6}, v_{3}^{2}=\frac{1}{6}, v^{3}=\frac{1}{6} .
$$

We find the root lattice of $S U(3) \times G_{2}^{2}$ to be the correct one. We choose for the twists acting on the lattice basis:

$$
\begin{aligned}
& Q_{1} e_{1}=-e_{1}, \quad Q_{1} e_{2}=-e_{2}, \quad Q_{1} e_{3}=e_{3}, \quad Q_{1} e_{4}=e_{4}, \\
& Q_{1} e_{5}=-e_{5}, \quad Q_{1} e_{6}=-e_{6}, \\
& Q_{2} e_{1}=-e_{2}, \quad Q_{2} e_{2}=e_{1}+e_{2}, \quad Q_{2} e_{3}=2 e_{3}+3 e_{4}, \quad Q_{2} e_{4}=-e_{3}-e_{4}, \\
& Q_{2} e_{5}=-2 e_{5}-3 e_{6}, \quad Q_{2} e_{6}=e_{5}+e_{6} .
\end{aligned}
$$

Here, the $Q_{2}$-twist on $e_{5}, e_{6}$ is minus the usual Coxeter-twist on $S U(3)$. The twists reproduce the correct eigenvalues and the conditions $Q_{1}^{2}=1, Q_{2}^{6}=1$. The combined twist $Q_{3}$ has the form

$$
\begin{aligned}
& Q_{3} e_{1}=e_{2}, \quad Q_{3} e_{2}=-e_{1}-e_{2}, \\
& Q_{3} e_{3}=2 e_{3}+3 e_{4}, \quad Q_{3} e_{4}=-e_{3}-e_{4}, \\
& Q_{3} e_{5}=2 e_{5}+3 e_{6}, \quad Q_{3} e_{6}=-e_{5}-e_{6},
\end{aligned}
$$

and also reproduces the required eigenvalues. We require the metric to be invariant under all three twists, i.e. we impose the three conditions $Q_{i}^{T} g Q_{i}=g, \quad i=1,2,3$. This leads to the following solution:

$$
g=\left(\begin{array}{cccccc}
R_{1}^{2} & -\frac{1}{2} R_{1}^{2} & 0 & 0 & 0 & 0 \\
-\frac{1}{2} R_{1}^{2} & R_{1}^{2} & 0 & 0 & 0 & 0 \\
0 & 0 & R_{3}^{2} & -\frac{1}{2} R_{3}^{2} & 0 & 0 \\
0 & 0 & -\frac{1}{2} R_{3}^{2} & \frac{1}{3} R_{3}^{2} & 0 & 0 \\
0 & 0 & 0 & 0 & R_{5}^{2} & -\frac{1}{2} R_{5}^{2} \\
0 & 0 & 0 & 0 & -\frac{1}{2} R_{5}^{2} & \frac{1}{3} R_{5}^{2} .
\end{array}\right)
$$

The solution for $b$ matches the pattern:

$$
b=\left(\begin{array}{cccccc}
0 & b_{1} & 0 & 0 & 0 & 0 \\
-b_{1} & 0 & 0 & 0 & 0 & 0 \\
0 & 0 & 0 & b_{2} & 0 & 0 \\
0 & 0 & -b_{2} & 0 & 0 & 0 \\
0 & 0 & 0 & 0 & 0 & -b_{3} \\
0 & 0 & 0 & 0 & -b_{3} & 0
\end{array}\right),
$$


we therefore know to have three Kähler moduli whereas the complex structure is completely fixed. For the complex structure we get

$$
\begin{aligned}
& d z^{1}=3^{1 / 4}\left(d x^{1}+e^{-2 \pi i / 3} d x^{2}\right), \\
& d z^{2}=d x^{3}+\frac{1}{\sqrt{3}} e^{10 \pi i / 12} d x^{4}, \\
& d z^{3}=d x^{5}+\frac{1}{\sqrt{3}} e^{10 \pi i / 12} d x^{6} .
\end{aligned}
$$

Examination of the Kähler form yields

$$
\begin{aligned}
\mathcal{T}^{1} & =\frac{\sqrt{3}}{2} R_{1}^{2}+i b_{1}, \\
\mathcal{T}^{2} & =\frac{1}{2 \sqrt{3}} R_{3}^{2}+i b_{2}, \\
\mathcal{T}^{3} & =\frac{1}{2 \sqrt{3}} R_{5}^{2}+i b_{3}
\end{aligned}
$$

\section{A.8. $\mathbf{Z}_{6} \times \mathbf{Z}_{6}$-orbifold}

The two twists act on the complex coordinate as follows:

$$
\begin{aligned}
& Q_{1}: z^{i} \longrightarrow e^{2 \pi i v_{1}^{i}} z^{i} \quad, \quad v_{1}^{1}=\frac{1}{6}, v_{1}^{2}=0, v_{1}^{3}=-\frac{1}{6}, \\
& Q_{2}: z^{i} \longrightarrow e^{2 \pi i v_{2}^{i}} z^{i} \quad, \quad v_{2}^{1}=0, v_{2}^{2}=\frac{1}{6}, v_{2}^{3}=-\frac{1}{6} .
\end{aligned}
$$

The combined twist $Q_{3}=Q_{2} Q_{1}$ is a $Z_{6-I}$-twist:

$$
Q_{3}: z^{i} \longrightarrow e^{2 \pi i v_{3}^{i}} z^{i} \quad, \quad v_{3}^{1}=\frac{1}{6}, v_{3}^{2}=\frac{1}{6}, v^{3}=-\frac{2}{6} .
$$

We find the root lattice of $G_{2} \times G_{2} \times G_{2}$ to be the correct one. We choose for the twists acting on the lattice basis:

$$
\begin{aligned}
& Q_{1} e_{1}=2 e_{1}+3 e_{2}, \quad Q_{1} e_{2}=-e_{1}-e_{2}, \quad Q_{1} e_{3}=e_{3}, \quad Q_{1} e_{4}=e_{4}, \\
& Q_{1} e_{5}=2 e_{5}+3 e_{6}, \quad Q_{1} e_{6}=-e_{5}-e_{6}, \\
& Q_{2} e_{1}=e_{1}, \quad Q_{2} e_{2}=e_{2}, \quad Q_{2} e_{3}=2 e_{3}+3 e_{4}, \quad Q_{2} e_{4}=-e_{3}-e_{4}, \\
& Q_{2} e_{5}=2 e_{5}+3 e_{6}, \quad Q_{2} e_{6}=-e_{5}-e_{6} .
\end{aligned}
$$

The twists reproduce the correct eigenvalues and the conditions $Q_{1}^{6}=1, Q_{2}^{6}=1$. The combined twist $Q_{3}$ has the form

$$
\begin{aligned}
& Q_{3} e_{1}=2 e_{1}+3 e_{3}, \quad Q_{3} e_{2}=-e_{1}-e_{2}, \\
& Q_{3} e_{3}=2 e_{3}+3 e_{4}, \quad Q_{3} e_{4}=-e_{3}-e_{4}, \\
& Q_{3} e_{5}=e_{5}+3 e_{6}, \quad Q_{3} e_{6}=-e_{5}-2 e_{6}
\end{aligned}
$$


where the twist on $e_{5}, e_{6}$ is twice the Coxeter-twist on $G_{2} . Q_{3}$ also reproduces the required eigenvalues. We require the metric to be invariant under all three twists, i.e. we impose the three conditions $Q_{i}^{T} g Q_{i}=g, \quad i=1,2,3$. This leads to the following solution:

$$
g=\left(\begin{array}{cccccc}
R_{1}^{2} & -\frac{1}{2} R_{1}^{2} & 0 & 0 & 0 & 0 \\
-\frac{1}{2} R_{1}^{2} & \frac{1}{3} R_{1}^{2} & 0 & 0 & 0 & 0 \\
0 & 0 & R_{3}^{2} & -\frac{1}{2} R_{3}^{2} & 0 & 0 \\
0 & 0 & -\frac{1}{2} R_{3}^{2} & \frac{1}{3} R_{3}^{2} & 0 & 0 \\
0 & 0 & 0 & 0 & R_{5}^{2} & -\frac{1}{2} R_{5}^{2} \\
0 & 0 & 0 & 0 & -\frac{1}{2} R_{5}^{2} & \frac{1}{3} R_{5}^{2} .
\end{array}\right)
$$

The solution for $b$ matches the pattern:

$$
b=\left(\begin{array}{cccccc}
0 & b_{1} & 0 & 0 & 0 & 0 \\
-b_{1} & 0 & 0 & 0 & 0 & 0 \\
0 & 0 & 0 & b_{2} & 0 & 0 \\
0 & 0 & -b_{2} & 0 & 0 & 0 \\
0 & 0 & 0 & 0 & 0 & -b_{3} \\
0 & 0 & 0 & 0 & -b_{3} & 0
\end{array}\right)
$$

we therefore know to have three Kähler moduli whereas the complex structure is completely fixed. For the complex structure we get

$$
\begin{aligned}
& d z^{1}=3^{1 / 4}\left(d x^{1}+\frac{1}{\sqrt{3}} e^{10 \pi i / 12} d x^{2}\right) \\
& d z^{2}=3^{1 / 4}\left(d x^{3}+\frac{1}{\sqrt{3}} e^{10 \pi i / 12} d x^{4}\right) \\
& d z^{3}=3^{1 / 4}\left(d x^{5}+\frac{1}{\sqrt{3}} e^{-10 \pi i / 12} d x^{6}\right) .
\end{aligned}
$$

Examination of the Kähler form yields

$$
\begin{aligned}
\mathcal{T}^{1} & =\frac{1}{2 \sqrt{3}} R_{1}^{2}+i b_{1} \\
\mathcal{T}^{2} & =\frac{1}{2 \sqrt{3}} R_{3}^{2}+i b_{2} \\
\mathcal{T}^{3} & =\frac{1}{2 \sqrt{3}} R_{5}^{2}+i b_{3}
\end{aligned}
$$

\section{Appendix B. Flux solutions for $\mathbf{Z}_{N}$ and $\mathbf{Z}_{N} \times \mathbf{Z}_{M^{-}}$-orbifolds}

In this appendix, we present the flux solutions of the remaining orbifolds we have considered in this paper. 


\section{B.1. Invariant Fluxes}

\begin{tabular}{|l|ccccccccc|}
\hline$G_{3}$ & $\mathbf{Z}_{3}$ & $\mathbf{Z}_{4}$ & $\mathbf{Z}_{6-I}$ & $\mathbf{Z}_{6-I I}$ & $\mathbf{Z}_{7}$ & $\mathbf{Z}_{8-I}$ & $\mathbf{Z}_{8-I I}$ & $\mathbf{Z}_{12-I}$ & $\mathbf{Z}_{12-I I}$ \\
\hline$d z^{1} \wedge d z^{2} \wedge d z^{3}$ & + & + & + & + & + & + & + & + & + \\
$d \bar{z}^{1} \wedge d z^{2} \wedge d z^{3}$ & - & - & - & - & - & - & - & - & - \\
$d z^{1} \wedge d \bar{z}^{2} \wedge d z^{3}$ & - & - & - & - & - & - & - & - & - \\
$d z^{1} \wedge d z^{2} \wedge d \bar{z}^{3}$ & - & + & - & + & - & - & + & - & + \\
$d z^{1} \wedge d \bar{z}^{2} \wedge d \bar{z}^{3}$ & - & - & - & - & - & - & - & - & - \\
$d \bar{z}^{1} \wedge d z^{2} \wedge d \bar{z}^{3}$ & - & - & - & - & - & - & - & - & - \\
$d \bar{z}^{1} \wedge d \bar{z}^{2} \wedge d z^{3}$ & - & + & - & + & - & - & + & - & + \\
$d \bar{z}^{1} \wedge d \bar{z}^{2} \wedge d \bar{z}^{3}$ & + & + & + & + & + & + & + & + & + \\
\hline
\end{tabular}

Table 4: Allowed 3-form fluxes for point group $\mathbf{Z}_{N}$

\begin{tabular}{|l|clllllll|}
\hline$G_{3}$ & $\mathbf{Z}_{2} \times \mathbf{Z}_{2}$ & $\mathbf{Z}_{3} \times \mathbf{Z}_{3}$ & $\mathbf{Z}_{2} \times \mathbf{Z}_{4}$ & $\mathbf{Z}_{4} \times \mathbf{Z}_{4}$ & $\mathbf{Z}_{2} \times \mathbf{Z}_{6-I}$ & $\mathbf{Z}_{2} \times \mathbf{Z}_{6-I I}$ & $\mathbf{Z}_{3} \times \mathbf{Z}_{6}$ & $\mathbf{Z}_{6} \times \mathbf{Z}_{6}$ \\
\hline$d z^{1} \wedge d z^{2} \wedge d z^{3}$ & + & + & + & + & + & + & + & + \\
$d \bar{z}^{1} \wedge d z^{2} \wedge d z^{3}$ & + & - & + & - & + & - & - & - \\
$d z^{1} \wedge d \bar{z}^{2} \wedge d z^{3}$ & + & - & - & - & - & - & - & - \\
$d z^{1} \wedge d z^{2} \wedge d \bar{z}^{3}$ & + & - & - & - & - & - & - & - \\
$d z^{1} \wedge d \bar{z}^{2} \wedge d \bar{z}^{3}$ & + & - & + & - & + & - & - & - \\
$d \bar{z}^{1} \wedge d z^{2} \wedge d \bar{z}^{3}$ & + & - & - & - & - & - & - & - \\
$d \bar{z}^{1} \wedge d \bar{z}^{2} \wedge d z^{3}$ & + & - & - & - & - & - & - & - \\
$d \bar{z}^{1} \wedge d \bar{z}^{2} \wedge d \bar{z}^{3}$ & + & + & + & + & + & + & + & + \\
\hline
\end{tabular}

Table 5: Allowed 3-form fluxes for point group $\mathbf{Z}_{M} \times \mathbf{Z}_{N}$

The remaining 12 fluxes of the form $d z^{a} \wedge d \bar{z}^{a} \wedge d z^{b}$ and $d z^{a} \wedge d \bar{z}^{a} \wedge d \bar{z}^{b}$, respectively are always projected out and therefore do not appear in the tables. Note, that we have for completeness also listed the orbifold groups $\Gamma \in\left\{\mathbf{Z}_{4}, \mathbf{Z}_{8-I}, \mathbf{Z}_{8-I I}, \mathbf{Z}_{12-I I}\right\}$, whose tadpoles may only be cancelled in the more general orbifold setups with discrete torsion or vector structure.

\section{B.2. $\mathbf{Z}_{3}$-orbifold}

On this orbifold, only the $(3,0)$ - and $(3,0)$-components survive, so the flux takes in complex notation the form

$$
\frac{1}{(2 \pi)^{2} \alpha^{\prime}} G_{3}=A_{0} \omega_{A_{0}}+B_{0} \omega_{B_{0}}
$$


We choose the free constants such that $d z^{1}=d x^{1}+e^{2 \pi i / 3} d x^{2}, d z^{2}=d x^{3}+e^{2 \pi i / 3} d x^{4}$ and $d z^{3}=d x^{5}+e^{2 \pi i / 3} d x^{6}$, as suggested in appendix A. The $(3,0)$-form on this orbifold takes the form

$$
\omega_{A_{0}}=\alpha_{0}+\beta_{0}+e^{2 \pi i / 3}\left(\alpha_{1}+\alpha_{2}+\alpha_{3}\right)+e^{2 \pi i / 6}\left(\beta_{1}+\beta_{2}+\beta_{3}\right) .
$$

$\omega_{B_{0}}$ is the complex conjugate of the above. For the complex coefficients we find

$$
\begin{aligned}
& A_{0}=\frac{1}{\sqrt{3}}\left\{-e^{2 \pi i / 12} a^{1}+e^{-2 \pi i / 12} b_{1}-i S\left[e^{2 \pi i / 12} c^{1}-e^{-2 \pi i / 12} d_{1}\right]\right\}, \\
& B_{0}=\frac{1}{\sqrt{3}}\left\{-e^{-2 \pi i / 12} a^{1}+e^{2 \pi i / 12} b_{1}+i S\left[e^{-2 \pi i / 12} c^{1}-e^{2 \pi i / 12} d_{1}\right]\right\} .
\end{aligned}
$$

Expressed in the real 3-forms, the flux takes the form

$$
\begin{aligned}
\frac{1}{(2 \pi)^{2} \alpha^{\prime}} G_{3}= & \left(-a^{1}+b_{1}+i S\left(-c^{1}+d_{1}\right)\right)\left(\alpha_{0}+\beta^{0}\right) \\
& +\left(a^{1}+i S c^{1}\right)\left(\alpha_{1}+\alpha_{2}+\alpha_{3}\right)+\left(b_{1}+i S d_{1}\right)\left(\beta^{1}+\beta^{2}+\beta^{3}\right) .
\end{aligned}
$$

\section{B.3. $\mathbf{Z}_{6-I}-$ orbifold}

Again, only the $(3,0)$ - and $(3,0)$-components survive, so the flux takes in complex notation the form

$$
\frac{1}{(2 \pi)^{2} \alpha^{\prime}} G_{3}=A_{0} \omega_{A_{0}}+B_{0} \omega_{B_{0}}
$$

The $G_{2}^{2} \times S U(3)$-lattice

The $(3,0)$-form on this orbifold takes the form

$$
\begin{aligned}
\omega_{A_{0}}= & \alpha_{0}+\frac{1}{\sqrt{3}} e^{5 \pi i / 6}\left(\alpha_{1}+\alpha_{2}\right)+e^{2 \pi i / 3} \alpha_{3}+\frac{1}{3} e^{2 \pi i / 6} \beta_{0} \\
& +\frac{i}{\sqrt{3}}\left(\beta_{1}+\beta_{2}\right)+\frac{1}{3} e^{2 \pi i / 3} \beta_{3} .
\end{aligned}
$$

$\omega_{B_{0}}$ is the complex conjugate of the above. For the complex coefficients we find

$$
\begin{aligned}
& A_{0}=\frac{1}{\sqrt{3}}\left(e^{2 \pi i / 12} a^{0}-i \sqrt{3} b_{0}+i S\left[e^{2 \pi i / 12} c^{0}-i \sqrt{3} d_{0}\right]\right), \\
& B_{0}=\frac{1}{\sqrt{3}}\left(e^{-2 \pi i / 12} a^{0}+i \sqrt{3} b_{0}-i S\left[e^{-2 \pi i / 12} c^{0}+i \sqrt{3} d_{0}\right]\right) .
\end{aligned}
$$

Expressed in the real 3-forms, the flux takes the form

$$
\begin{aligned}
\frac{1}{(2 \pi)^{2} \alpha^{\prime}} G_{3}= & \left(a^{0}+i S c^{0}\right) \alpha_{0}+\frac{1}{3}\left(-2 a^{0}+3 b_{0}-i S\left[2 c^{0}-3 d_{0}\right]\right)\left(\alpha_{1}+\alpha_{2}\right) \\
& +\left(-a^{0}+3 b_{0}-i S\left(c^{0}-3 d_{0}\right)\right) \alpha_{3}+\left(b_{0}+i S d_{0}\right) \beta^{0} \\
& +\frac{1}{3}\left(-a^{0}+6 b_{0}-i S\left[c^{0}-6 d_{0}\right]\right)\left(\beta^{1}+\beta^{2}\right)+\left(-a^{0}+3 b_{0}-i S\left[c^{0}-3 d_{0}\right]\right) \beta^{3} .
\end{aligned}
$$


In order for all the real coefficients to be integer, $a^{0}, b_{0}, c^{0}$ and $d_{0}$ should be chosen to be multiples of 3 .

The $G_{2} \times(S U(3))^{2}$-lattice with generalized Coxeter element

The $(3,0)$-form on this orbifold takes the form

$$
\begin{aligned}
\omega_{A_{0}}= & 2\left(\alpha_{0}+\frac{1}{\sqrt{3}} e^{5 \pi i / 6} \alpha_{1}+e^{2 \pi i / 3} \alpha_{2}-e^{2 \pi i / 6} \alpha_{3}+\frac{1}{\sqrt{3}} e^{5 \pi i / 6} \beta_{0}\right. \\
& \left.-\beta_{1}-\frac{1}{\sqrt{3}} e^{2 \pi i / 6} \beta_{2}+\frac{i}{\sqrt{3}} \beta_{3}\right) .
\end{aligned}
$$

$\omega_{B_{0}}$ is the complex conjugate of the above. For the complex coefficients we find

$$
\begin{aligned}
& A_{0}=-\frac{1}{2}\left(e^{2 \pi i / 3} a^{0}+\sqrt{3} b_{0}-i S\left[e^{5 \pi i / 6} c^{0}+\sqrt{3} d_{0}\right]\right), \\
& B_{0}=-\frac{1}{2}\left(e^{-2 \pi i / 3} a^{0}+\sqrt{3} b_{0}+i S\left[e^{-5 \pi i / 6} c^{0}+\sqrt{3} d_{0}\right]\right) .
\end{aligned}
$$

Expressed in the real 3-forms, the flux takes the form

$$
\begin{aligned}
\frac{1}{(2 \pi)^{2} \alpha^{\prime}} G_{3}= & \left(a^{0}+i S c^{0}\right) \alpha_{0}+\left(a^{1}+i S C^{1}\right) \alpha_{1}+\left(a^{0}+3 a^{1}+i S\left[c^{0}+3 c^{1}\right]\right) \alpha_{2} \\
& +\left(-2 a^{0}-3 a^{1}-i S\left(2 c^{0}+3 c^{1}\right)\right) \alpha_{3}+\left(a^{1}+i S c^{1}\right) \beta^{0} \\
& +\left(-a^{0}-i S c^{0}\right) \beta^{1}+\left(-a^{0}-a^{1}-i S\left[c^{0}+c^{1}\right]\right) \beta^{2} \\
& +\left(a^{0}+2 a^{1}+i S\left[c^{0}+2 c^{1}\right]\right) \beta^{3} .
\end{aligned}
$$

\section{B.4. $\mathbf{Z}_{6-I I}$-orbifold}

This is a case with one complex structure modulus left unfixed. Therefore one $(2,1)-$ and one $(1,2)$-component survive and the flux takes the form

$$
\frac{1}{(2 \pi)^{2} \alpha^{\prime}} G_{3}=A_{0} \omega_{A_{0}}+A_{3} \omega_{A_{3}}+B_{0} \omega_{B_{0}}+B_{3} \omega_{B_{3}}
$$

The $(S U(6)) \times S U(2)$-lattice

The $(3,0)$-form on this orbifold takes the form

$$
\begin{aligned}
\omega_{A_{0}}= & \frac{1}{6}\left\{\left(-3 i \alpha_{0}+(i+\sqrt{3})\left(\alpha_{1}+\alpha_{2}-\delta_{6}\right)+(-i+\sqrt{3})\left(\beta_{3}-\gamma_{2}-\gamma_{3}\right)\right.\right. \\
& -i\left(\gamma_{1}+\gamma_{4}-\delta_{5}\right)-U\left[3 i \alpha_{3}-3 i \beta_{0}+2 \sqrt{3} \beta_{1}+\sqrt{3} \beta_{2}\right. \\
& \left.\left.-3 i \gamma_{5}+3 i \gamma_{6}+\sqrt{3}\left(\delta_{1}-\delta_{2}-2 \delta_{3}-\delta_{4}\right)\right]\right\} .
\end{aligned}
$$


The one $(2,1)$-form surviving the twist takes the form

$$
\begin{aligned}
\omega_{A_{3}}= & \frac{1}{6}\left\{\left(3 i \alpha_{0}+(-i+\sqrt{3})\left(\alpha_{1}+\alpha_{2}-\delta_{6}\right)+(i+\sqrt{3})\left(\beta_{3}-\gamma_{2}-\gamma_{3}\right)\right.\right. \\
& +i\left(\gamma_{1}+\gamma_{4}-\delta_{5}\right)-U\left[-3 i \alpha_{3}+3 i \beta_{0}+2 \sqrt{3} \beta_{1}+\sqrt{3} \beta_{2}\right. \\
& \left.\left.+3 i \gamma_{5}-3 i \gamma_{6}+\sqrt{3}\left(\delta_{1}-\delta_{2}-2 \delta_{3}-\delta_{4}\right)\right]\right\} .
\end{aligned}
$$

$\omega_{B_{0}}$ and $\omega_{B_{3}}$ are the complex conjugate of the above. For the complex coefficients we find

$$
\begin{aligned}
A_{0}= & \frac{1}{2 \operatorname{Im} \mathcal{U}^{3}}\left\{-b_{0}+\frac{i \sqrt{3}}{2} b_{1}-S\left(\frac{\sqrt{3}}{2} d_{1}+i d_{0}\right)\right. \\
& \left.+U\left[-a_{0}+i\left(\frac{1}{\sqrt{3}} a_{0}+\sqrt{3} a_{1}\right)-S\left(\frac{1}{\sqrt{3}} c_{0}+\sqrt{3} c_{1}+i c_{0}\right)\right]\right\}, \\
B_{0}= & \frac{1}{2 \operatorname{Im} \mathcal{U}^{3}}\left\{-b_{0}-\frac{i \sqrt{3}}{2} b_{1}+S\left(\frac{\sqrt{3}}{2} d_{1}-i d_{0}\right)\right. \\
& \left.+\bar{U}\left[-a_{0}-i\left(\frac{1}{\sqrt{3}} a_{0}+\sqrt{3} a_{1}\right)+S\left(\frac{1}{\sqrt{3}} c_{0}+\sqrt{3} c_{1}-i c_{0}\right)\right]\right\}, \\
A_{3}= & \frac{1}{2 \operatorname{Im} \mathcal{U}^{3}}\left\{b_{0}+\frac{i \sqrt{3}}{2} b_{1}-S\left(\frac{\sqrt{3}}{2} d_{1}-i d_{0}\right)\right. \\
& \left.+U\left[a_{0}+i\left(\frac{1}{\sqrt{3}} a_{0}+\sqrt{3} a_{1}\right)-S\left(\frac{1}{\sqrt{3}} c_{0}+\sqrt{3} c_{1}-i c_{0}\right)\right]\right\}, \\
B_{3}= & \frac{1}{2 \operatorname{Im} \mathcal{U}^{3}}\left\{b_{0}-\frac{i \sqrt{3}}{2} b_{1}+S\left(\frac{\sqrt{3}}{2} d_{1}+i d_{0}\right)\right. \\
& \left.+\bar{U}\left[a_{0}-i\left(\frac{1}{\sqrt{3}} a_{0}+\sqrt{3} a_{1}\right)+S\left(\frac{1}{\sqrt{3}} c_{0}+\sqrt{3} c_{1}+i c_{0}\right)\right]\right\},
\end{aligned}
$$

Expressed in the real 3-forms, the flux takes the form

$$
\begin{aligned}
\frac{1}{(2 \pi)^{2} \alpha^{\prime}} G_{3}= & \frac{1}{3}\left(a_{0}+i S c_{0}\right)\left(3 \alpha_{0}+2 \beta_{3}+\gamma_{1}-2 \gamma_{2}-2 \gamma_{3}+\gamma_{4}-\delta_{5}\right) \\
& +\left(b_{0}+i S d_{0}\right)\left(-\alpha_{3}+\beta_{0}+\gamma_{5}-\gamma_{6}\right) \\
& +\frac{1}{2}\left(b_{1}+i S d_{1}\right)\left(2 \beta_{1}+\beta_{2}+\delta_{1}-\delta_{2}-2 \delta_{3}-\delta_{4}\right) \\
& +\left(a_{1}+i S c_{1}\right)\left(\alpha_{1}+\alpha_{2}+\beta_{3}-\gamma_{2}-\gamma_{3}-\delta_{6}\right) .
\end{aligned}
$$

The $S U(3) \times S O(8)$-lattice 
The $(3,0)$-form on this orbifold takes the form

$$
\begin{aligned}
\omega_{A_{0}}= & -\left(2+\mathcal{U}^{3}\right) \alpha_{0}+\left(e^{-2 \pi i / 6}+\mathcal{U}^{3}\right) \alpha_{1}-\left(1+2 \mathcal{U}^{3}\right) \alpha_{2}+e^{2 \pi i / 3}\left(2+\mathcal{U}^{3}\right) \alpha_{3} \\
& +\left(\mathcal{U}^{3}+e^{2 \pi i / 3}\left(1+\mathcal{U}^{3}\right)\right) \beta^{0}+e^{2 \pi i / 3}\left(1+2 \mathcal{U}^{3}\right) \beta_{1}-e^{2 \pi i /}\left(1+\mathcal{U}^{3}\right) \beta_{2} \\
& +\left(-1+e^{2 \pi i / 3} \mathcal{U}^{3}\right) \beta^{3}+\left(1+e^{2 \pi i / 6}\right)\left(1+\mathcal{U}^{3}\right) \gamma_{2}+\left(-1+\mathcal{U}^{3}\right) \gamma_{4} \\
& +\left(-1+e^{2 \pi i / 3}\right)\left(1+\mathcal{U}^{3}\right) \delta^{2}+e^{2 \pi i / 3}\left(-1-\mathcal{U}^{3}\right) \delta^{4} .
\end{aligned}
$$

The one $(2,1)$-form surviving the twist takes the form

$$
\begin{aligned}
\omega_{A_{3}}= & -\left(2+\overline{\mathcal{U}}^{3}\right) \alpha_{0}+\left(e^{-2 \pi i / 6}+\overline{\mathcal{U}}^{3}\right) \alpha_{1}-\left(1+2 \overline{\mathcal{U}}^{3}\right) \alpha_{2}+e^{2 \pi i / 3}\left(2+\overline{\mathcal{U}}^{3}\right) \alpha_{3} \\
& +\left(\mathcal{U}^{3}+e^{2 \pi i / 3}\left(1+\overline{\mathcal{U}}^{3}\right)\right) \beta^{0}+e^{2 \pi i / 3}\left(1+2 \overline{\mathcal{U}}^{3}\right) \beta_{1}-e^{2 \pi i /}\left(1+\overline{\mathcal{U}}^{3}\right) \beta_{2} \\
& +\left(-1+e^{2 \pi i / 3} \overline{\mathcal{U}}^{3}\right) \beta^{3}+\left(1+e^{2 \pi i / 6}\right)\left(1+\overline{\mathcal{U}}^{3}\right) \gamma_{2}+\left(-1+\overline{\mathcal{U}}^{3}\right) \gamma_{4} \\
& +\left(-1+e^{2 \pi i / 3}\right)\left(1+\overline{\mathcal{U}}^{3}\right) \delta^{2}+e^{2 \pi i / 3}\left(-1-\overline{\mathcal{U}}^{3}\right) \delta^{4} .
\end{aligned}
$$

$\omega_{B_{0}}$ and $\omega_{B_{3}}$ are the complex conjugate of the above. For the complex coefficients we find

$$
\begin{aligned}
A_{0}= & \frac{i}{6 \operatorname{Im} \mathcal{U}^{3}}\left\{e^{2 \pi i / 6}\left(1+2 \overline{\mathcal{U}}^{3}\right) a^{1}+\left(1+e^{2 \pi i / 6} \overline{\mathcal{U}}^{3}\right) a_{2}-\left(e^{-2 \pi i / 6}+\overline{\mathcal{U}}^{3}\right) b_{1}-\left(1+2 \overline{\mathcal{U}}^{3}\right) b_{2}\right. \\
& \left.\left.-i S\left[-e^{2 \pi i / 6}\left(1+2 \overline{\mathcal{U}}^{3}\right) c_{1}+\left(1+e^{2 \pi i / 6} \overline{\mathcal{U}}^{3}\right) c_{2}-\left(e^{-2 \pi i / 6}+\overline{\mathcal{U}}^{3}\right) d_{1}+\left(1+2 \overline{\mathcal{U}}^{3}\right) d_{2}\right)\right]\right\} \\
B_{0}= & \frac{-i}{6 \operatorname{Im} \mathcal{U}^{3}}\left\{e^{-2 \pi i / 6}\left(1+2 \mathcal{U}^{3}\right) a^{1}+\left(1+e^{-2 \pi i / 6} \mathcal{U}^{3}\right) a_{2}-\left(e^{2 \pi i / 6}+\mathcal{U}^{3}\right) b_{1}-\left(1+2 \mathcal{U}^{3}\right) b_{2}\right. \\
& \left.\left.-i S\left[-e^{-2 \pi i / 6}\left(1+2 \mathcal{U}^{3}\right) c_{1}-\left(1+e^{-2 \pi i / 6} \mathcal{U}^{3}\right) c_{2}+\left(e^{2 \pi i / 6}+\mathcal{U}^{3}\right) d_{1}+\left(1+2 \mathcal{U}^{3}\right) d_{2}\right)\right]\right\} \\
A_{3}= & \frac{i}{6 \operatorname{Im} \mathcal{U}^{3}}\left\{-e^{2 \pi i / 6}\left(1+2 \mathcal{U}^{3}\right) a^{1}-\left(1+e^{2 \pi i / 6} \mathcal{U}^{3}\right) a_{2}+\left(e^{-2 \pi i / 6}+\mathcal{U}^{3}\right) b_{1}+\left(1+2 \mathcal{U}^{3}\right) b_{2}\right. \\
& \left.\left.-i S\left[e^{2 \pi i / 6}\left(1+2 \mathcal{U}^{3}\right) c_{1}+\left(1+e^{2 \pi i / 6} \mathcal{U}^{3}\right) c_{2}-\left(e^{-2 \pi i / 6}+\mathcal{U}^{3}\right) d_{1}-\left(1+2 \mathcal{U}^{3}\right) d_{2}\right)\right]\right\} \\
B_{3}= & \frac{-i}{6 \operatorname{Im} \mathcal{U}^{3}}\left\{-e^{-2 \pi i / 6}\left(1+2 \overline{\mathcal{U}}^{3}\right) a^{1}-\left(1+e^{-2 \pi i / 6} \overline{\mathcal{U}}^{3}\right) a_{2}+\left(e^{2 \pi i / 6}+\overline{\mathcal{U}}^{3}\right) b_{1}+\left(1+2 \overline{\mathcal{U}}^{3}\right) b_{2}\right. \\
& \left.\left.-i S\left[-e^{-2 \pi i / 6}\left(1+2 \overline{\mathcal{U}}^{3}\right) c_{1}+\left(1+e^{-2 \pi i / 6} \overline{\mathcal{U}}^{3}\right) c_{2}+\left(e^{2 \pi i / 6}+\overline{\mathcal{U}}^{3}\right) d_{1}-\left(1+2 \overline{\mathcal{U}}^{3}\right) d_{2}\right)\right]\right\} .
\end{aligned}
$$

Expressed in the real 3-forms, the flux takes the form

$$
\begin{aligned}
\frac{1}{(2 \pi)^{2} \alpha^{\prime}} G_{3}= & \left(-a^{1}+b_{1}+2 b_{2}+i S\left(-c^{1}+d_{1}+2 d_{2}\right)\right) \alpha_{0}+\left(a^{1}+i S c^{1}\right) \alpha_{1} \\
& +\left(a^{2}+i S c^{2}\right) \alpha_{2}+\left(2 a^{1}+a^{2}-b_{1}-b_{2}+i S\left(2 c^{1}+c^{2}-d_{1}-d_{2}\right)\right) \alpha_{3} \\
& +\left(-a^{1}-a^{2}+b_{1}+b_{2}+i S\left(-c^{1}-c^{2}+b_{1}+b_{2}\right)\right) \beta^{0}+\left(b_{1}+i S d_{1}\right) \beta^{1} \\
& +\left(b_{2}+i S d_{2}\right) \beta^{2}+\left(b_{1}+b_{2}+i S\left(d_{1}+d_{2}\right)\right) \beta^{3}-\left(a^{2}+b^{2}+i S\left(c^{2}+d^{2}\right)\right) \gamma_{2} \\
& +\left(-a^{1}-a^{2}+b_{1}+2 b_{2}+i S\left(-c^{1}-c^{2}+d_{1}+2 d^{2}\right)\right) \gamma_{4} \\
& +\left(-a^{1}+b_{1}+b_{2}+i S\left(-c^{1}+d_{1}+d_{2}\right)\right) \delta_{2} \\
& +\left(2 a^{1}+a^{2}-b_{2}+i S\left(2 c^{1}+c^{2}-d_{2}\right)\right) \delta_{4} .
\end{aligned}
$$




\section{B.5. $\mathbf{Z}_{7}$-orbifold}

In section 2 we learned that only the $(3,0)$ - and the $(0,3)$-flux component survive the $Z_{7}$-twist, so

$$
\frac{1}{(2 \pi)^{2} \alpha^{\prime}} G_{3}=A_{0} \omega_{A_{0}}+B_{0} \omega_{B_{0}} .
$$

In appendix A, we calculated the complex structure. With this, we find for the $(3,0)$-form:

$$
\begin{aligned}
\omega_{A_{0}}= & -i \sqrt{7} \alpha_{0}+\frac{1}{2}(7+i \sqrt{7}) \alpha_{1}+i \sqrt{7} \alpha_{2}-i \sqrt{7} \alpha_{3}-i \sqrt{7} \beta_{0}+i \sqrt{7} \beta_{1}-i \sqrt{7} \beta_{2} \\
& +\frac{1}{2}(7-i \sqrt{7}) \beta_{3}-i \sqrt{7} \gamma_{1}+i \sqrt{7} \gamma_{2}+\frac{1}{2}(-7+i \sqrt{7}) \gamma_{3}-i \sqrt{7} \gamma_{4}+(7+i \sqrt{7}) \gamma_{5} \\
& -(7-i \sqrt{7}) \gamma_{6}-i \sqrt{7} \delta_{1}-(7+i \sqrt{7}) \delta_{2}-i \sqrt{7} \delta_{3}+(1-i \sqrt{7}) \delta_{4}+i \sqrt{7} \delta_{5}-(7+i \sqrt{7}) \delta_{6} .
\end{aligned}
$$

$\omega_{B_{0}}$ is simply the complex conjugate of the above. It is possible to express the two complex coefficients of the 3-form flux through four of the real coefficients which we may choose freely. The other real coefficients are constrained by the form of the flux. For the complex coefficients we find

$$
\begin{aligned}
& A_{0}=\frac{1}{14}\left[(1+i \sqrt{7}) a^{0}+2 a^{1}-i S\left((1+i \sqrt{7}) c^{0}+2 c^{1}\right)\right] \\
& B_{0}=\frac{1}{14}\left[(1-i \sqrt{7}) a^{0}+2 a^{1}+i S\left((1-i \sqrt{7}) c^{0}+2 c^{1}\right)\right] .
\end{aligned}
$$

Expressed in real coordinates, the flux takes the form

$$
\begin{aligned}
\frac{1}{(2 \pi)^{2} \alpha^{\prime}} G_{3}= & \left(a^{0}+i S c^{0}\right) \alpha_{0}+\left(a^{1}+i S c^{1}\right) \alpha_{1}+\left(-a^{0}-i S c^{0}\right) \alpha_{2}+\left(a^{0}+i S c^{0}\right) \alpha_{3} \\
& +\left(a^{0}+i S c^{0}\right) \beta^{0}+\left(-a^{0}-i S c^{0}\right) \beta^{1}+\left(a^{0}+i S c^{0}\right) \beta^{2} \\
& \left.+\left(a^{0}+a^{1}+i S\left(c^{0}+c^{1}\right)\right)\right) \beta^{3}+\left(a^{0}+i S c^{0}\right) \gamma_{1}+\left(-a^{0}+i S c^{0}\right) \gamma_{2} \\
& +\left(-a^{0}-a^{1}-i S\left(c^{0}+c^{1}\right)\right) \gamma_{3}+\left(a^{0}+i S c^{0}\right) \gamma_{4} \\
& +\left(a^{1}+i S c^{1}\right) \gamma_{5}+\left(-a^{0}-a^{1}-i S\left(c^{0}+c^{1}\right)\right) \gamma_{6}-\left(a^{1}+i S c^{1}\right) \delta^{6} \\
& -\left(-a^{0}-a^{1}-i S\left(c^{1}+c^{1}\right)\right) \delta^{4}-\left(a^{0}+i S c^{0}\right) \delta^{5}-\left(a^{1}+i S c^{1}\right) \delta^{2} \\
& -\left(-a^{0}-i S c^{0}\right) \delta^{3}-\left(a^{0}+i S c^{0}\right) \delta^{1}
\end{aligned}
$$

\section{B.6. $\mathbf{Z}_{12-I^{-} \text {orbifold }}$}

This is another case where only the $(3,0)$ - and the $(0,3)$-flux component survive the twist, so

$$
\frac{1}{(2 \pi)^{2} \alpha^{\prime}} G_{3}=A_{0} \omega_{A_{0}}+B_{0} \omega_{B_{0}} .
$$


The $S U(3) \times F_{4}$-lattice

Using the complex structure found in appendix A, we find for the $(3,0)$-form:

$$
\begin{aligned}
\omega_{A_{0}}= & -e^{2 \pi i / 12} \alpha_{0}+e^{-2 \pi i / 12} \alpha_{1}+\frac{1}{\sqrt{2}} e^{2 \pi i / 8}\left(\sqrt{3} e^{-2 \pi i / 6} \alpha_{2}+e^{-2 \pi i / 12} \alpha_{3}-i \sqrt{3} \beta_{0}\right. \\
& +\sqrt{3} e^{-2 \pi i / 12} \beta_{1}+(1-i) \beta_{2}-\sqrt{3} e^{2 \pi i / 6} \beta_{3}+(1+\sqrt{3}) \gamma_{3} \\
& \left.-\frac{1}{\sqrt{2}} e^{2 \pi i / 8}(1+i(2+\sqrt{3})) \delta^{5}\right) .
\end{aligned}
$$

$\omega_{B_{0}}$ is the complex conjugate of the above. For the complex coefficients we find

$$
\begin{aligned}
& A_{0}=\frac{1}{\sqrt{2}} e^{5 \pi i / 12} a^{1}+\frac{1}{\sqrt{3}} e^{2 \pi i / 6} b_{1}+i S\left[\frac{1}{\sqrt{2}} e^{5 \pi i / 12} c^{1}+\frac{1}{\sqrt{3}} e^{2 \pi i / 6} d_{1}\right], \\
& B_{0}=\frac{1}{\sqrt{2}} e^{-5 \pi i / 12} a^{1}+\frac{1}{\sqrt{3}} e^{-2 \pi i / 6} b_{1}-i S\left[\frac{1}{\sqrt{2}} e^{-5 \pi i / 12} c^{1}+\frac{1}{\sqrt{3}} e^{-2 \pi i / 6} d_{1}\right] .
\end{aligned}
$$

Expressed in real coordinates, the flux takes the form

$$
\begin{aligned}
\frac{1}{(2 \pi)^{2} \alpha^{\prime}} G_{3}= & -3\left(b_{1}+i S d_{1}\right) \alpha_{0}+\left(a^{1}+i S c^{1}\right) \alpha_{1}+\frac{1}{3}\left(a^{1}+3 b_{1}+i S\left(c^{1}+3 d_{1}\right)\right) \alpha_{2} \\
& +\left(-a^{1}+\frac{2}{\sqrt{3}} b_{1}+i S\left(c^{1}+\frac{2}{\sqrt{3}} d_{1}\right)\right) \alpha_{3}+\frac{1}{3}\left(a^{1}+i S c^{1}\right) \beta^{0}+\left(b_{1}+i S d_{1}\right) \beta^{1} \\
& +\frac{1}{3}\left(a^{1}+3 b_{1}+i S\left(c^{1}+3 d_{1}\right)\right) \beta^{2}+\left(\sqrt{3} a^{1}-b_{1}+i S\left(\sqrt{3} c^{1}-d_{1}\right)\right) \beta^{3} .
\end{aligned}
$$

Here we have again some factors of $\sqrt{3}$, which in this case can only be got rid of by further constraining the real coefficients and fixing $S . S=i$ and $a^{1}=c^{1}, b_{1}=d_{1}$ does the job.

\section{B.7. $\mathbf{Z}_{3} \times \mathbf{Z}_{3}$-orbifold}

Only the $(3,0)-$ and the $(0,3)$-flux component survive the twist, so

$$
\frac{1}{(2 \pi)^{2} \alpha^{\prime}} G_{3}=A_{0} \omega_{A_{0}}+B_{0} \omega_{B_{0}} .
$$

Using the complex structure found in chapter 3 , we find for the $(3,0)$-form:

$$
\omega_{A_{0}}=\alpha_{0}+e^{2 \pi i / 3} \alpha_{1}+e^{2 \pi i / 3} \alpha_{2}+e^{-2 \pi i / 3} \alpha_{3}+e^{2 \pi i / 3} \beta_{0}-\beta_{1}-\beta_{2}+e^{2 \pi i / 3} \beta_{3} .
$$

$\omega_{B_{0}}$ is the complex conjugate of the above. For the complex coefficients we find

$$
\begin{aligned}
& A_{0}=\frac{1}{\sqrt{3}}\left\{-i a^{1}+e^{5 \pi i / 6} b_{1}+i S\left[-i c^{1}+e^{5 \pi i / 6} d_{1}\right]\right\}, \\
& B_{0}=\frac{1}{\sqrt{3}}\left\{i a^{1}+e^{-5 \pi i / 6} b_{1}-i S\left[i c^{1}+e^{-5 \pi i / 6} d_{1}\right]\right\} .
\end{aligned}
$$


Expressed in real coordinates, the flux takes the form

$$
\begin{aligned}
\frac{1}{(2 \pi)^{2} \alpha^{\prime}} G_{3}= & \left(-b_{1}-i S d_{1}\right) \alpha_{0}+\left(a^{1}+i S c^{1}\right)\left(\alpha_{1}+\alpha_{2}\right)+\left(-a^{1}+b_{1}+i S\left(-c^{1}+d_{1}\right)\right) \alpha_{3} \\
& \left.+\left(a^{1}+i S c^{1}\right) \beta^{0}+\left(b_{1}+i S d_{1}\right)\left(\beta^{1}+\beta^{2}\right)+\left(a^{1}-b_{1}+i S\left(c^{1}-d_{1}\right)\right)\right) \beta^{3} .
\end{aligned}
$$

\section{B.8. $\mathbf{Z}_{2} \times \mathbf{Z}_{6}$-orbifold}

This is a case with one complex structure modulus left unfixed. Therefore one $(2,1)-$ and one $(1,2)$-component survive and the flux takes the form

$$
\frac{1}{(2 \pi)^{2} \alpha^{\prime}} G_{3}=A_{0} \omega_{A_{0}}+A_{3} \omega_{A_{3}}+B_{0} \omega_{B_{0}}+B_{3} \omega_{B_{3}} .
$$

The $(3,0)$-form on this orbifold takes the form

$$
\begin{aligned}
\omega_{A_{0}}= & \alpha_{0}+\frac{1}{\sqrt{3}} e^{5 \pi i / 6} \alpha_{2}+e^{2 \pi i / 3} \alpha_{3}+\frac{i}{\sqrt{3}} \beta^{1} \\
& +\mathcal{U}^{3}\left[\alpha_{1}-\frac{i}{\sqrt{3}} \beta_{0}+e^{-2 \pi i / 6} \beta_{2}+\frac{1}{\sqrt{3}} e^{-\pi i / 6} \beta_{3}\right] .
\end{aligned}
$$

The one $(2,1)$-form surviving the twist takes the form

$$
\begin{aligned}
\omega_{A_{3}}= & \alpha_{0}+\frac{1}{\sqrt{3}} e^{-5 \pi i / 6} \alpha_{2}+e^{-2 \pi i / 3} \alpha_{3}+\frac{1}{\sqrt{3}} e^{2 \pi i / 3} \beta^{1} \\
& +\mathcal{U}^{3}\left[\alpha_{1}+\frac{1}{\sqrt{3}} e^{\pi i / 6} \beta_{0}+e^{2 \pi i / 6} \beta_{2}+\frac{1}{\sqrt{3}} e^{-\pi i / 6} \beta_{3}\right] .
\end{aligned}
$$

$\omega_{B_{0}}$ and $\omega_{B_{3}}$ are the complex conjugate of the above. For the complex coefficients we find

$$
\begin{aligned}
A_{0}= & \frac{1}{\mathcal{U}^{3}}\left\{-b_{0}+\frac{1}{\sqrt{3}} e^{\pi i / 6} b_{2}+\mathcal{U}^{3}\left[-\frac{i}{\sqrt{3}} a_{0}-e^{2 \pi i / 6} a_{2}+i S\left(\frac{-i}{\sqrt{3}} c_{0}-e^{2 \pi i / 6} c_{2}\right.\right.\right. \\
& \left.\left.\left.-\frac{1}{\sqrt{3}} e^{\pi i / 6} c^{3}+d_{1}\right)\right]\right\}, \\
B_{0}= & \frac{1}{\overline{\mathcal{U}}^{3}}\left\{-b_{0}+\frac{1}{\sqrt{3}} e^{-\pi i / 6} b_{2}+\overline{\mathcal{U}}^{3}\left[\frac{i}{\sqrt{3}} a_{0}-e^{-2 \pi i / 6} a_{2}-i S\left(\frac{i}{\sqrt{3}} c_{0}+e^{-2 \pi i / 6} c_{2}\right.\right.\right. \\
& \left.\left.\left.+\frac{1}{\sqrt{3}} e^{-\pi i / 6} c^{3}-d_{1}\right)\right]\right\}, \\
A_{3}= & \frac{1}{\mathcal{U}^{3}}\left\{b_{0}-\frac{1}{\sqrt{3}} e^{\pi i / 6} b_{2}+\mathcal{U}^{3}\left[\frac{1}{\sqrt{3}} e^{-\pi i / 6} a_{0}+e^{-2 \pi i / 6} a_{2}+i S\left(-\frac{1}{\sqrt{3}} e^{-\pi i / 6} c_{0}\right.\right.\right. \\
& \left.\left.\left.+e^{2 \pi i / 3} c_{2}-\frac{1}{\sqrt{3}} e^{\pi i / 6} c^{3}+d_{1}\right)\right]\right\} \\
B_{3}= & \frac{1}{\overline{\mathcal{U}}^{3}}\left\{b_{0}-\frac{1}{\sqrt{3}} e^{-\pi i / 6} b_{2}+\overline{\mathcal{U}}^{3}\left[\frac{1}{\sqrt{3}} e^{\pi i / 6} a_{0}+e^{2 \pi i / 6} a_{2}+i S\left(-\frac{1}{\sqrt{3}} e^{\pi i / 6} c_{0}\right.\right.\right. \\
& \left.\left.\left.+e^{-2 \pi i / 3} c_{2}-\frac{1}{\sqrt{3}} e^{-\pi i / 6} c^{3}+d_{1}\right)\right]\right\}
\end{aligned}
$$


Expressed in the real 3-forms, the flux takes the form

$$
\begin{aligned}
\frac{1}{(2 \pi)^{2} \alpha^{\prime}} G_{3}= & \left(a^{0}+i S c^{0}\right) \alpha_{0}+\left[\operatorname{Re} \mathcal{U}^{3}\left(a^{0}+i S c^{0}\right)\right. \\
& \left.+\sqrt{3} \operatorname{Im} \mathcal{U}^{3}\left(a^{0}+2 a^{2}+i S\left(c^{0}+2 c^{2}\right)\right)\right] \alpha_{1} \\
& +\left(a^{2}+i S c^{2}\right) \alpha_{2}+\frac{1}{\left|\mathcal{U}^{3}\right|^{2}}\left[\sqrt{3} \operatorname{Im} \mathcal{U}^{3}\left(-2 b_{0}+b_{2}\right)+i\left(\operatorname{Im} \mathcal{U}^{3}\right)^{2} S c^{3}\right) \\
& \left.-\operatorname{Re} \mathcal{U}^{3}\left(b_{2}-i \operatorname{Re} \mathcal{U}^{3} S c^{3}\right)\right] \alpha_{3}+\left[b_{0}-i \frac{1}{3} S\left(\sqrt{3} \operatorname{Im} \mathcal{U}^{3}\left(2 c^{3}-3 d_{1}\right)\right.\right. \\
& \left.+3 \operatorname{Re} \mathcal{U}^{3} d_{1}\right] \beta^{0}-\frac{1}{\left|\mathcal{U}^{3}\right|^{2}}\left[\operatorname{Re} \mathcal{U}^{3} b_{0}+\operatorname{Im} \mathcal{U}^{3}\left(\sqrt{3} b_{0}-\frac{2}{\sqrt{3}} b_{2}\right)\right. \\
& \left.-i\left|\mathcal{U}^{3}\right|^{2} S d_{1}\right] \beta^{1}+\left[b_{2}+i S\left(\operatorname{Re} \mathcal{U}^{3} c^{3}+\sqrt{3} \operatorname{Im} \mathcal{U}^{3}\left(c^{3}-2 d_{1}\right)\right)\right] \beta^{2} \\
& +\left[\operatorname{Re} \mathcal{U}^{3}\left(-a^{2}-i S c^{2}\right)+\frac{1}{\sqrt{3}} \operatorname{Im} \mathcal{U}^{3}\left(2 a^{0}+3 a^{2}+i S\left(2 c^{0}+3 c_{2}\right)\right)\right] \beta^{3}
\end{aligned}
$$

This again presents only a valid flux for certain choices of $\mathcal{U}^{3}$ like for example $e^{2 \pi i / 6}$ or $\frac{1}{\sqrt{3}} e^{\pi i / 6}$.

\section{B.9. $\mathbf{Z}_{2} \times \mathbf{Z}_{6^{\prime}}$-orbifold}

Again, the flux is of the form

$$
\frac{1}{(2 \pi)^{2} \alpha^{\prime}} G_{3}=A_{0} \omega_{A_{0}}+B_{0} \omega_{B_{0}} .
$$

Using the complex structure found in appendix A, we find for the $(3,0)$-form:

$\omega_{A_{0}}=\alpha_{0}+e^{-2 \pi i / 3} \alpha_{1}+\frac{1}{\sqrt{3}} e^{5 \pi i / 6}\left(\alpha_{2}+\alpha_{3}\right)-\frac{1}{3} \beta_{0}+\frac{1}{3} e^{2 \pi i / 3} \beta_{1}+\frac{1}{\sqrt{3}} e^{-5 \pi i / 6}\left(\beta_{2}+\beta_{3}\right)$.

$\omega_{B_{0}}$ is the complex conjugate of the above. For the complex coefficients we find

$$
\begin{aligned}
& \left.A_{0}=\frac{1}{\sqrt{3}} e^{5 \pi i / 6} a^{1}+\sqrt{3} e^{-5 \pi i / 6} b_{1}-i S\left[\frac{1}{\sqrt{3}} e^{5 \pi i / 6} c^{1}+\sqrt{3} e^{-5 \pi i / 6} d_{1}\right]\right\}, \\
& \left.B_{0}=\frac{1}{\sqrt{3}} e^{-5 \pi i / 6} a^{1}+\sqrt{3} e^{5 \pi i / 6} b_{1}+i S\left[\frac{1}{\sqrt{3}} e^{-5 \pi i / 6} c^{1}+\sqrt{3} e^{5 \pi i / 6} d_{1}\right]\right\} .
\end{aligned}
$$

Expressed in real coordinates, the flux takes the form

$$
\begin{aligned}
\frac{1}{(2 \pi)^{2} \alpha^{\prime}} G_{3}= & -\left(a^{1}+3 b_{1}+i S\left(c^{1}+3 d_{1}\right)\right) \alpha_{0}+\left(a^{1}+i S c^{1}\right) \alpha_{1} \\
& +\frac{1}{3}\left(a^{1}+6 b_{1}+i S\left(c^{1}+6 d_{1}\right)\right) \alpha_{2} \\
& +\frac{1}{3}\left(a^{1}+6 b_{1}+i S\left(c^{1}+6 d_{1}\right)\right) \alpha_{3}+\frac{1}{3}\left(a^{1}+3 b_{1}+i S\left(c^{1}+3 d_{1}\right)\right) \beta^{0} \\
& +\left(b_{1}+i S d_{1}\right) \beta^{1}+\frac{1}{3}\left(2 a^{1}+3 b_{1}+i S\left(2 c^{1}+3 d_{1}\right)\left(\beta^{2}+\beta^{3}\right) .\right.
\end{aligned}
$$




\section{B.10. $\mathbf{Z}_{3} \times \mathbf{Z}_{6}$-orbifold}

Again, the flux is of the form

$$
\frac{1}{(2 \pi)^{2} \alpha^{\prime}} G_{3}=A_{0} \omega_{A_{0}}+B_{0} \omega_{B_{0}} .
$$

Using the complex structure found in chapter 2, we find for the $(3,0)$-form:

$$
\begin{aligned}
\omega_{A_{0}}= & \alpha_{0}+e^{2 \pi i / 3} \alpha_{1}+\frac{1}{\sqrt{3}} e^{5 \pi i / 6} \alpha_{2}+\frac{1}{\sqrt{3}} e^{-5 \pi i / 6} \alpha_{3} \\
& \left.+\frac{1}{3} e^{2 \pi i / 3} \beta_{0}-2 \beta_{1}+\frac{1}{\sqrt{3}}\right) e^{5 \pi i / 6} \beta_{2}+\frac{i}{\sqrt{3}} \beta_{3} .
\end{aligned}
$$

$\omega_{B_{0}}$ is the complex conjugate of the above. For the complex coefficients we find

$$
\begin{aligned}
& \left.A_{0}=-\frac{i}{\sqrt{3}} a^{1}+\sqrt{3} e^{5 \pi i / 6} b_{1}+i S\left[-\frac{i}{\sqrt{3}} c^{1}+\sqrt{3} e^{5 \pi i / 6} d_{1}\right]\right\}, \\
& \left.B_{0}=\frac{i}{\sqrt{3}} a^{1}+\sqrt{3} e^{-5 \pi i / 6} b_{1}-i S\left[\frac{i}{\sqrt{3}} c^{1}+\sqrt{3} e^{-5 \pi i / 6} d_{1}\right]\right\} .
\end{aligned}
$$

Expressed in real coordinates, the flux takes the form

$$
\begin{aligned}
\frac{1}{(2 \pi)^{2} \alpha^{\prime}} G_{3}= & 3\left(-b_{1}-i S d_{1}\right) \alpha_{0}+\left(a^{1}+i S c^{1}\right) \alpha_{1}+\frac{1}{3}\left(a^{1}+3 b_{1}+i S\left(c^{1}+3 d_{1}\right)\right) \alpha_{2} \\
& +\frac{1}{3}\left(-a^{1}+6 b_{1}-i S\left(c^{1}-6 d_{1}\right)\right) \alpha_{3}+\frac{1}{3}\left(a^{1}+i S c^{1}\right) \beta^{0}+\left(b_{1}+i S d_{1}\right) \beta^{1} \\
& +\frac{1}{3}\left(a^{1}+3 b_{1}+i S\left(c^{1}+3 d_{1}\right) \beta^{2}+\frac{1}{3}\left(2 a^{1}-3 b_{1}+i S\left(2 c^{1}-3 d_{1}\right)\right)\right) \beta^{3} .
\end{aligned}
$$

\section{B.11. $\mathbf{Z}_{6} \times \mathbf{Z}_{6}$-orbifold}

Again, the flux is of the form

$$
\frac{1}{(2 \pi)^{2} \alpha^{\prime}} G_{3}=A_{0} \omega_{A_{0}}+B_{0} \omega_{B_{0}} .
$$

Using the complex structure found in appendix A, we find for the $(3,0)$-form:

$$
\begin{aligned}
\omega_{A_{0}}= & \alpha_{0}+\frac{1}{\sqrt{3}} e^{5 \pi i / 6}\left(\alpha_{1}+\alpha_{2}\right)+\frac{1}{\sqrt{3}} e^{-5 \pi i / 6} \alpha_{3}-\frac{1}{3 \sqrt{3}} e^{5 \pi i / 6} \beta_{0} \\
& -\frac{1}{3}\left(\beta_{1}+\beta_{2}\right)+\frac{1}{\sqrt{3}} e^{5 \pi i / 6} \beta_{3} .
\end{aligned}
$$

$\omega_{B_{0}}$ is the complex conjugate of the above. For the complex coefficients we find

$$
\begin{aligned}
& \left.A_{0}=-i \sqrt{3} a^{1}+3 e^{2 \pi i / 3} b_{1}+i S\left[-i \sqrt{3} c^{1}+3 e^{2 \pi i / 3} d_{1}\right]\right\}, \\
& \left.B_{0}=i \sqrt{3} a^{1}+3 e^{-2 \pi i / 3} b_{1}-i S\left[i \sqrt{3} c^{1}+3 e^{-2 \pi i / 3} d_{1}\right]\right\} .
\end{aligned}
$$


Expressed in real coordinates, the flux takes the form

$$
\begin{aligned}
\frac{1}{(2 \pi)^{2} \alpha^{\prime}} G_{3}= & -3\left(b_{1}+i S d_{1}\right) \alpha_{0}+\left(a^{1}+i S c^{1}\right)\left(\alpha_{1}+\alpha_{2}\right)+\left(-a^{1}+3 b_{1}+i S\left(-c^{1}+3 d_{1}\right)\right) \alpha_{3} \\
& +\frac{1}{3}\left(a^{1}+i S c^{1}\right) \beta^{0}+\left(b_{1}+i S d_{1}\right)\left(\beta^{1}+\beta^{2}\right)+\left(a^{1}-b_{1}+i S\left(c^{1}-d_{1}\right)\right) \beta^{3} .
\end{aligned}
$$

\section{Appendix C. Cartan matrices of the relevant Lie groups}

$$
A=\left(\begin{array}{cccccccc}
2 & -1 & 0 & . & . & . & 0 & 0 \\
-1 & 2 & -1 & . & . & . & 0 & 0 \\
0 & -1 & 2 & -1 & . & . & 0 & 0 \\
. & . & . & . & . & . & . & . \\
0 & 0 & 0 & . & . & -1 & 2 & -1 \\
0 & 0 & 0 & . & . & 0 & -1 & 2
\end{array}\right)
$$

$S O(2 n)$

$$
A=\left(\begin{array}{cccccccc}
2 & -1 & 0 & . & . & . & 0 & 0 \\
-1 & 2 & -1 & . & . & . & 0 & 0 \\
0 & -1 & 2 & -1 & . & . & 0 & 0 \\
. & . & . & . & . & . & . & . \\
0 & 0 & 0 & . & . & -1 & 2 & -2 \\
0 & 0 & 0 & . & . & 0 & -1 & 2
\end{array}\right)
$$

$G_{2}$

$$
A=\left(\begin{array}{cc}
2 & -3 \\
-1 & 2
\end{array}\right)
$$

$F_{4}$

$$
A=\left(\begin{array}{cccc}
2 & -1 & 0 & 0 \\
-1 & 2 & -2 & 0 \\
0 & -1 & 2 & -1 \\
0 & 0 & -1 & 2
\end{array}\right)
$$

$E_{6}$

$$
A=\left(\begin{array}{cccccc}
2 & -1 & 0 & 0 & 0 & 0 \\
-1 & 2 & -1 & 0 & 0 & 0 \\
0 & -1 & 2 & -1 & 0 & -1 \\
0 & 0 & -1 & 2 & -1 & 0 \\
0 & 0 & 0 & -1 & 2 & 0 \\
0 & 0 & -1 & 0 & 0 & 2
\end{array}\right)
$$

\section{Appendix D. Anti-symmetric tensor and Kähler moduli for heterotic orbifolds}

For completeness we shall present for the two cosets $\frac{S U(2,2)}{S U(2) \times S U(2) \times U(1)} \times \frac{S U(1,1)}{U(1)}$ and $\frac{S U(3,3)}{S U(3) \times S U(3)}$ the parameterization of the Kähler moduli on the heterotic side. 
D.1. Coset space $\frac{S U(2,2)}{S U(2) \times S U(2) \times U(1)} \times \frac{S U(1,1)}{U(1)}$

On the heterotic side, the twist-invariant 2-form $B_{2}$

$$
B_{2}=b_{1} d x^{1} \wedge d y^{1}+b_{2} d x^{2} \wedge d y^{2}+b_{5} d x^{3} \wedge d y^{3}+b_{3}\left(d x^{1} \wedge d x^{2}+d y^{1} \wedge d y^{2}\right)+b_{4}\left(d x^{1} \wedge d y^{2}-d y^{1} \wedge d x^{2}\right)
$$

is relevant. The twist invariant 2-forms are the Hodge-dual of the invariant 4-forms, given in (2.38). Hence, there is a direct correspondence between the expansion of $C_{4}$ and $B_{2}$. W.r.t. the complex coordinates (2.35) the 2-form $B_{2}$ becomes:

$$
B_{2}=i b_{1} d z^{1} \wedge d \bar{z}^{1}+i b_{2} d z^{2} \wedge d \bar{z}^{2}+i b_{5} d z^{3} \wedge d \bar{z}^{3}+\left(b_{3}-i b_{4}\right) d \bar{z}^{1} \wedge d z^{2}+\left(b_{3}+i b_{4}\right) d z^{1} \wedge d \bar{z}^{2}
$$

We read off the definition of the (complexified) Kähler moduli $\mathcal{T}^{i}$ from expanding the form $J+i B$ w.r.t. a basis $\omega_{j}, j=1, \ldots, h_{(1,1)}$ for $H^{2}\left(X_{6}, \mathbf{Z}\right)$, i.e. $J+i B=\mathcal{T}^{j} \omega_{j}$. Hence we would conclude:

$$
\begin{aligned}
& \mathcal{T}^{1}=g_{11}+i b_{1} \quad, \quad \mathcal{T}^{2}=g_{22}+i b_{2} \quad, \quad \mathcal{T}^{5}=\operatorname{Re}\left(\mathcal{T}^{5}\right)+i b_{5}, \\
& \mathcal{T}^{3}=g_{13}+i b_{4} \quad, \quad \mathcal{T}^{4}=g_{14}+i b_{3} \text {. }
\end{aligned}
$$

The Kähler potential $K_{\mathcal{K}}$ takes the same form as in (2.41). In fact, it may be checked, that from (2.41) the correct metric for the background $g, b$ (given in Eqs. (2.34) and (D.1)), i.e.

$$
\frac{1}{8} \operatorname{Tr}\left(d g^{-1} d g\right)+\frac{1}{8} \operatorname{Tr}\left(g^{-1} d b g^{-1} d b\right)=\sum_{j, k} \frac{\partial^{2} K_{\mathcal{K}}}{\partial \mathcal{T}^{j} \partial \overline{\mathcal{T}}^{k}} d \mathcal{T}^{j} d \overline{\mathcal{T}}^{k}
$$

follows.

\section{D.2. Coset space $\frac{S U(3,3)}{S U(3) \times S U(3)}$}

On the heterotic side we consider the twist-invariant 2-form $B_{2}$

$$
\begin{aligned}
B_{2} & =b_{1} d x^{1} \wedge d y^{1}+b_{2} d x^{2} \wedge d y^{2}+b_{3} d x^{3} \wedge d y^{3} \\
& +b_{4}\left(d x^{1} \wedge d x^{2}-d y^{1} \wedge d x^{2}+d y^{1} \wedge d y^{2}\right)+b_{5}\left(d x^{1} \wedge d y^{2}-d y^{1} \wedge d x^{2}\right) \\
& +b_{6}\left(d x^{1} \wedge d x^{3}-d y^{1} \wedge d x^{3}+d y^{1} \wedge d y^{3}\right)+b_{7}\left(d x^{1} \wedge d y^{3}-d y^{1} \wedge d x^{3}\right) \\
& +b_{8}\left(d x^{2} \wedge d x^{3}-d y^{2} \wedge d x^{3}+d y^{2} \wedge d y^{3}\right)+b_{9}\left(d x^{2} \wedge d y^{3}-d y^{2} \wedge d x^{3}\right)
\end{aligned}
$$

which becomes

$$
\begin{aligned}
B_{2} & =i \sqrt{3} b_{1} d z^{1} \wedge d \bar{z}^{1}+i \sqrt{3} b_{2} d z^{2} \wedge d \bar{z}^{2}+i \sqrt{3} b_{3} d z^{3} \wedge d \bar{z}^{3} \\
& -i \sqrt{3}\left(\rho b_{4}-b_{5}\right) d z^{1} \wedge d \bar{z}^{2}-i \sqrt{3}\left(\rho b_{6}-b_{7}\right) d z^{1} \wedge d \bar{z}^{3}-i \sqrt{3}\left(\rho b_{8}-b_{9}\right) d z^{2} \wedge d \bar{z}^{3} \\
& -i \sqrt{3}\left(\bar{\rho} b_{4}-b_{5}\right) d z^{2} \wedge d \bar{z}^{1}-i \sqrt{3}\left(\bar{\rho} b_{6}-b_{7}\right) d z^{3} \wedge d \bar{z}^{1}-i \sqrt{3}\left(\bar{\rho} b_{8}-b_{9}\right) d z^{3} \wedge d \bar{z}^{2}
\end{aligned}
$$


in complex coordinates (2.48). On the heterotic side, we decompose the form $B+i J$ w.r.t. the integral (twist-invariant) basis of $H^{2}\left(X_{6}, \mathbf{Z}\right)$, i.e. $J+i B=\mathcal{T}^{j} \omega_{j}$ to read of the nine complexified Kähler moduli $\mathcal{T}^{i}$. This gives:

$$
\begin{array}{ll}
\mathcal{T}^{1}=\frac{\sqrt{3}}{2} g_{11}+i b_{1} \quad, \quad \mathcal{T}^{2}=\frac{\sqrt{3}}{2} g_{33}+i b_{2} \quad, \quad \mathcal{T}^{3}=\frac{\sqrt{3}}{2} g_{55}+i b_{3}, \\
\mathcal{T}^{4}=-\frac{1}{\sqrt{3}}\left(g_{13}+2 g_{14}\right)+i b_{4} \quad, \quad \mathcal{T}^{5}=\frac{1}{\sqrt{3}}\left(2 g_{13}+g_{14}\right)+i b_{5}, \\
\mathcal{T}^{6}=-\frac{1}{\sqrt{3}}\left(g_{15}+2 g_{16}\right)+i b_{6} \quad, \quad \mathcal{T}^{7}=\frac{1}{\sqrt{3}}\left(2 g_{15}+g_{16}\right)+i b_{7}, \\
\mathcal{T}^{8}=-\frac{1}{\sqrt{3}}\left(g_{35}+2 g_{36}\right)+i b_{8} \quad, \quad \mathcal{T}^{9}=\frac{1}{\sqrt{3}}\left(2 g_{35}+g_{36}\right)+i b_{9} .
\end{array}
$$

Again, the Kähler potential $K_{\mathcal{K}}$ assumes the same form as in (2.54). 


\section{References}

[1] E. Kiritsis, "D-branes in standard model building, gravity and cosmology," Fortsch. Phys. 52, 200 (2004) arXiv:hep-th/0310001;

D. Lüst, "Intersecting brane worlds: A path to the standard model?," Class. Quant. Grav. 21, S1399 (2004) arXiv:hep-th/0401156;

R. Blumenhagen, M. Cvetic, P. Langacker and G. Shiu, "Toward realistic intersecting D-brane models," arXiv:hep-th/0502005.

[2] S. Kachru and A.K. Kashani-Poor, "Moduli potentials in type IIA compactifications with RR and NS flux," JHEP 0503, 066 (2005) arXiv:hep-th/0411279.

[3] J.P. Derendinger, C. Kounnas, P.M. Petropoulos and F. Zwirner, "Superpotentials in IIA compactifications with general fluxes," Nucl. Phys. B 715, 211 (2005) arXiv:hepth/0411276].

[4] T.W. Grimm and J. Louis, "The effective action of type IIA Calabi-Yau orientifolds," Nucl. Phys. B 718, 153 (2005) arXiv:hep-th/0412277.

[5] G. Villadoro and F. Zwirner, " $\mathrm{N}=1$ effective potential from dual type-IIA D6/O6 orientifolds with general fluxes," arXiv:hep-th/0503169.

[6] O. DeWolfe, A. Giryavets, S. Kachru and W. Taylor, "Type IIA moduli stabilization," arXiv:hep-th/0505160.

[7] P.G. Camara, A. Font and L.E. Ibanez, "Fluxes, moduli fixing and MSSM-like vacua in a simple IIA orientifold," arXiv:hep-th/0506066.

[8] S. Gukov, C. Vafa and E. Witten, "CFT's from Calabi-Yau four-folds," Nucl. Phys. B 584, 69 (2000) [Erratum-ibid. B 608, 477 (2001)] [arXiv:hep-th/9906070;

T.R. Taylor and C. Vafa, "RR flux on Calabi-Yau and partial supersymmetry breaking," Phys. Lett. B 474, 130 (2000) arXiv:hep-th/9912152;

P. Mayr, "On supersymmetry breaking in string theory and its realization in brane worlds," Nucl. Phys. B 593, 99 (2001) arXiv:hep-th/0003198.

[9] G. Curio, A. Klemm, D. Lüst and S. Theisen, "On the vacuum structure of type II string compactifications on Calabi-Yau spaces with H-fluxes," Nucl. Phys. B 609, 3 (2001) arXiv:hep-th/0012213.

[10] S.B. Giddings, S. Kachru and J. Polchinski, "Hierarchies from fluxes in string compactifications," Phys. Rev. D 66, 106006 (2002) arXiv:hep-th/0105097.

[11] S. Kachru, M.B. Schulz and S. Trivedi, "Moduli stabilization from fluxes in a simple IIB orientifold," JHEP 0310, 007 (2003) arXiv:hep-th/0201028.

[12] P.G. Camara, L.E. Ibanez and A.M. Uranga, "Flux-induced SUSY-breaking soft terms," Nucl. Phys. B 689, 195 (2004) [arXiv:hep-th/0311241].

[13] M. Grana, T.W. Grimm, H. Jockers and J. Louis, "Soft supersymmetry breaking in Calabi-Yau orientifolds with D-branes and fluxes," Nucl. Phys. B 690, 21 (2004) arXiv:hep-th/0312232. 
[14] D. Lüst, S. Reffert and S. Stieberger, "Flux-induced soft supersymmetry breaking in chiral type IIB orientifolds with D3/D7-branes," Nucl. Phys. B 706, 3 (2005) arXiv:hep-th/0406092.

[15] P.G. Camara, L.E. Ibanez and A.M. Uranga, "Flux-induced SUSY-breaking soft terms on D7-D3 brane systems," Nucl. Phys. B 708, 268 (2005) [arXiv:hep-th/0408036.

[16] D. Lüst, S. Reffert and S. Stieberger, "MSSM with soft SUSY breaking terms from D7-branes with fluxes," Nucl. Phys. B 727, 264 (2005) arXiv:hep-th/0410074.

[17] A. Font and L.E. Ibanez, "SUSY-breaking soft terms in a MSSM magnetized D7-brane model," JHEP 0503, 040 (2005) arXiv:hep-th/0412150.

[18] D. Lüst, P. Mayr, S. Reffert and S. Stieberger, "F-theory flux, destabilization of orientifolds and soft terms on D7-branes," Nucl. Phys. B 732, 243 (2006) arXiv:hepth/0501139].

[19] S. Kachru, R. Kallosh, A. Linde and S.P. Trivedi, "De Sitter vacua in string theory," Phys. Rev. D 68, 046005 (2003) arXiv:hep-th/0301240].

[20] S. Kachru, R. Kallosh, A. Linde, J. Maldacena, L. McAllister and S.P. Trivedi, "Towards inflation in string theory," JCAP 0310, 013 (2003) arXiv:hep-th/0308055.

[21] M.R. Douglas, "The statistics of string / M theory vacua," JHEP 0305, 046 (2003) arXiv:hep-th/0303194.

[22] F. Denef and M.R. Douglas, "Distributions of flux vacua," JHEP 0405, 072 (2004) arXiv:hep-th/0404116;

B.S. Acharya, F. Denef and R. Valandro, "Statistics of M theory vacua," arXiv:hepth/0502060.

[23] R. Blumenhagen, F. Gmeiner, G. Honecker, D. Lust and T. Weigand, "The statistics of supersymmetric D-brane models," Nucl. Phys. B 713, 83 (2005) arXiv:hepth/0411173].

[24] F. Denef, M.R. Douglas and B. Florea, "Building a better racetrack," JHEP 0406, 034 (2004) arXiv:hep-th/0404257.

[25] L. Görlich, S. Kachru, P.K. Tripathy and S.P. Trivedi, "Gaugino condensation and nonperturbative superpotentials in flux arXiv:hep-th/0407130.

[26] P.K. Tripathy and S.P. Trivedi, "D3 Brane Action and Fermion Zero Modes in Presence of Background Flux," arXiv:hep-th/0503072.

[27] R. Kallosh, A.K. Kashani-Poor and A. Tomasiello, "Counting fermionic zero modes on M5 with fluxes," arXiv:hep-th/0503138.

[28] N. Saulina, "Topological constraints on stabilized flux vacua," arXiv:hep-th/0503125.

[29] P. Berglund and P. Mayr, "Non-perturbative superpotentials in F-theory and string duality," arXiv:hep-th/0504058.

[30] P.S. Aspinwall and R. Kallosh, "Fixing all moduli for M-theory on K3 x K3," arXiv:hep-th/0506014. 
[31] D. Lüst, S. Reffert, E. Scheidegger, W. Schulgin and S. Stieberger, "Moduli stabilization in type IIB orientifolds. II," arXiv:hep-th/0609013.

[32] F. Denef, M.R. Douglas, B. Florea, A. Grassi and S. Kachru, "Fixing All Moduli in a Simple F-Theory Compactification," arXiv:hep-th/0503124.

[33] G. Curio, A. Krause and D. Lüst, "Moduli stabilization in the heterotic / IIB discretuum," arXiv:hep-th/0502168.

[34] P. Breitenlohner and D.Z. Freedman, "Stability In Gauged Extended Supergravity," Annals Phys. 144, 249 (1982).

[35] K. Choi, A. Falkowski, H.P. Nilles, M. Olechowski and S. Pokorski, "Stability of flux compactifications and the pattern of supersymmetry breaking," JHEP 0411, 076 (2004) arXiv:hep-th/0411066.

[36] L.J. Dixon, J.A. Harvey, C. Vafa and E. Witten, "Strings On Orbifolds," Nucl. Phys. B 261, 678 (1985); "Strings On Orbifolds. 2," Nucl. Phys. B 274, 285 (1986).

[37] G. Aldazabal, A. Font, L.E. Ibanez and G. Violero, "D = 4, N = 1, type IIB orientifolds," Nucl. Phys. B 536, 29 (1998) arXiv:hep-th/9804026.

[38] G. Zwart, "Four-dimensional $\mathrm{N}=1 \mathrm{Z}(\mathrm{N}) \times \mathrm{Z}(\mathrm{M})$ orientifolds," Nucl. Phys. B 526, 378 (1998) arXiv:hep-th/9708040.

[39] R. Blumenhagen, J.P. Conlon and K. Suruliz, "Type IIA orientifolds on general supersymmetric Z(N) orbifolds," JHEP 0407, 022 (2004) arXiv:hep-th/0404254.

[40] D. Lüst, P. Mayr, R. Richter and S. Stieberger, "Scattering of gauge, matter, and moduli fields from intersecting branes," Nucl. Phys. B 696, 205 (2004) arXiv:hepth/0404134.

[41] I. Antoniadis, C. Bachas, C. Fabre, H. Partouche and T.R. Taylor, "Aspects of type I - type II - heterotic triality in four dimensions," Nucl. Phys. B 489, 160 (1997) arXiv:hep-th/9608012;

T.W. Grimm and J. Louis, "The effective action of $\mathrm{N}=1$ Calabi-Yau orientifolds," Nucl. Phys. B 699, 387 (2004) arXiv:hep-th/0403067;

H. Jockers and J. Louis, "The effective action of D7-branes in N = 1 Calabi-Yau orientifolds," Nucl. Phys. B 705, 167 (2005) [arXiv:hep-th/0409098; "D-terms and Fterms from D7-brane fluxes," Nucl. Phys. B 718, 203 (2005) arXiv:hep-th/0502059.

[42] S. Ferrara, C. Kounnas and M. Porrati, "General Dimensional Reduction Of TenDimensional Supergravity And Superstring," Phys. Lett. B 181, 263 (1986).

[43] M. Cvetic, J. Louis and B.A. Ovrut, "A String Calculation Of The Kahler Potentials For Moduli Of Z(N) Orbifolds," Phys. Lett. B 206, 227 (1988).

[44] L.E. Ibanez and D. Lüst, "Duality anomaly cancellation, minimal string unification and the effective low-energy Lagrangian of 4-D strings," Nucl. Phys. B 382, 305 (1992) arXiv:hep-th/9202046. 
[45] S. Ferrara and S. Theisen, "Moduli Spaces, Effective Actions And Duality Symmetry In String Compactifications," CERN-TH-5652-90 Based on lectures given at 3rd Hellenic Summer School, Corfu, Greece, Sep 13-23, 1989

[46] L.E. Ibanez, J. Mas, H.P. Nilles and F. Quevedo, "Heterotic Strings In Symmetric And Asymmetric Orbifold Backgrounds," Nucl. Phys. B 301, 157 (1988);

J. Erler and A. Klemm, "Comment on the generation number in orbifold compactifications," Commun. Math. Phys. 153, 579 (1993) arXiv:hep-th/9207111.

[47] A. Font, L.E. Ibanez and F. Quevedo, "Z(N) X Z(M) Orbifolds And Discrete Torsion," Phys. Lett. B 217, 272 (1989).

[48] M. Spalinski, "Duality transformations in twisted Narain compactifications," Nucl. Phys. B 377, 339 (1992). "On the discrete symmetry group of Narian orbifolds," Phys. Lett. B 275, 47 (1992).

[49] M. Marino, R. Minasian, G.W. Moore and A. Strominger, "Nonlinear instantons from supersymmetric p-branes," JHEP 0001, 005 (2000) arXiv:hep-th/9911206].

[50] A.R. Frey and J. Polchinski, "N $=3$ warped compactifications," Phys. Rev. D 65, 126009 (2002) arXiv:hep-th/0201029.

[51] R. Blumenhagen, D. Lüst and T.R. Taylor, "Moduli stabilization in chiral type IIB orientifold models with fluxes," Nucl. Phys. B 663, 319 (2003) arXiv:hep-th/0303016.

[52] J.F.G. Cascales and A.M. Uranga, "Chiral 4d N $=1$ string vacua with D-branes and NSNS and RR fluxes," JHEP 0305, 011 (2003) arXiv:hep-th/0303024.

[53] A. Font, "Z(N) orientifolds with flux," JHEP 0411, 077 (2004) arXiv:hep-th/0410206.

[54] J. Wess and J. Bagger, "Supersymmetry and supergravity," Princeton, USA: Univ. Pr. (1992) 259 p.

[55] K. Choi, A. Falkowski, H.P. Nilles and M. Olechowski, "Soft supersymmetry breaking in KKLT flux compactification," Nucl. Phys. B 718, 113 (2005) arXiv:hepth/0503216.

[56] F. Marchesano and G. Shiu, "Building MSSM flux vacua," JHEP 0411, 041 (2004) arXiv:hep-th/0409132.

[57] D. Robbins and S. Sethi, "A barren landscape," Phys. Rev. D 71, 046008 (2005) arXiv:hep-th/0405011.

[58] E. Witten, "Non-Perturbative Superpotentials In String Theory," Nucl. Phys. B 474, 343 (1996) arXiv:hep-th/9604030.

[59] K.A. Intriligator and N. Seiberg, "Lectures on supersymmetric gauge theories and electric-magnetic duality," Nucl. Phys. Proc. Suppl. 45BC, 1 (1996) arXiv:hepth/9509066.

[60] J.A. Casas, F. Gomez and C. Munoz, "Complete structure of Z(n) Yukawa couplings," Int. J. Mod. Phys. A 8, 455 (1993) arXiv:hep-th/9110060. 